July 23, 2015

\title{
Intracellular transport driven by cytoskeletal motors : General mechanisms and defects
}

\author{
C. Appert-Rolland ${ }^{\mathrm{a}, \mathrm{b}}$, M. Ebbinghaus $\mathrm{s}^{\mathrm{a}, \mathrm{b}, \mathrm{c}}$, L. Santen ${ }^{\mathrm{c}}$ \\ ${ }^{a}$ Univ. Paris-Sud, Laboratoire de Physique Théorique, Bât. 210, F-91405 Orsay Cedex, France \\ ${ }^{b}$ CNRS, LPT, UMR 8627, Bât 210, F-91405 Orsay Cedex, France \\ ${ }^{c}$ Fachrichtung Theoretische Physik, Universität des Saarlandes, D-66123 Saarbrücken, Germany
}

\begin{abstract}
Cells are the elementary units of living organisms, which are able to carry out many vital functions. These functions rely on active processes on a microscopic scale. Therefore, they are strongly out-of-equilibrium systems, which are driven by continuous energy supply. The tasks that have to be performed in order to maintain the cell alive require transportation of various ingredients, some being small, others being large. Intracellular transport processes are able to induce concentration gradients and to carry objects to specific targets. These processes cannot be carried out only by diffusion, as cells may be crowded, and quite elongated on molecular scales. Therefore active transport has to be organized.

The cytoskeleton, which is composed of three types of filaments (microtubules, actin and intermediate filaments), determines the shape of the cell, and plays a role in cell motion. It also serves as a road network for a special kind of vehicles, namely the cytoskeletal motors. These molecules can attach to a cytoskeletal filament, perform directed motion, possibly carrying along some cargo, and then detach.

It is a central issue to understand how intracellular transport driven by molecular motors is regulated. The interest for this type of question was enhanced when it was discovered that intracellular transport breakdown is one of the signatures of some neuronal diseases like the Alzheimer.

We give a survey of the current knowledge on microtubule based intracellular transport. Our review includes on the one hand an overview of biological facts, obtained from experiments, and on the other hand a presentation of some modeling attempts based on cellular automata. We present some background knowledge on the original and variants of the TASEP (Totally Asymmetric Simple Exclusion Process), before turning to more application oriented models. After addressing microtubule based transport in general, with a focus on in vitro experiments, and on cooperative effects in the transportation of large cargos by multiple motors, we concentrate on axonal transport, because of its relevance for neuronal diseases. Some important characteristics of axonal transport is that it takes place in a confined environment; Besides several types of motors are involved, that move in opposite directions. It is a challenge to understand how this bidirectional transport is organized. We review several features that could contribute to the efficiency of bidirectional transport in the axon, including in particular the role of motor-motor interactions and of the dynamics of the underlying microtubule network. Finally, we also discuss some open questions that may be relevant for future research in this field.
\end{abstract}

Keywords: Stochastic transport, intracellular transport, exclusion processes, molecular motors, cytoskeleton, dynamic networks

Email addresses: Cecile.Appert-Rolland@th.u-psud.fr (C. Appert-Rolland), ebbinghaus@lusi.uni-sb.de (M. Ebbinghaus), 1. santen@mx. uni-saarland.de (L. Santen)

Preprint submitted to Physics Reports 


\section{Contents}

1 Introduction $\quad 5$

2 Cytoskeleton filaments $\quad \mathbf{6}$

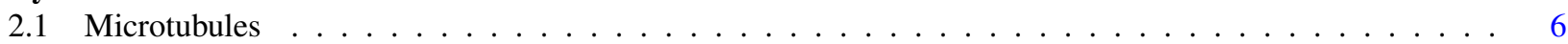

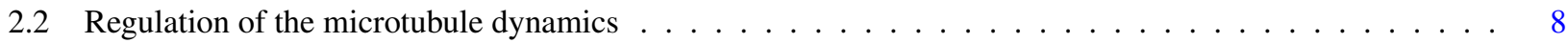

2.3 Modeling the dynamical instability: the two-state model . . . . . . . . . . . . . . . 9

2.4 Models with explicit description of the GTP cap . . . . . . . . . . . . . . . . . . 10

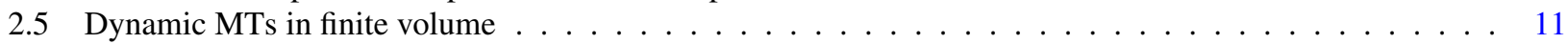

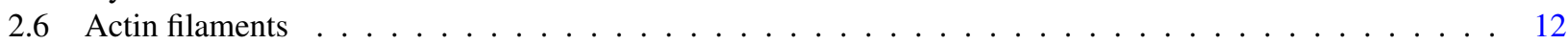

2.7 Treadmilling filaments . . . . . . . . . . . . . . . . . . . . . . . . 14

3 Molecular motors 15

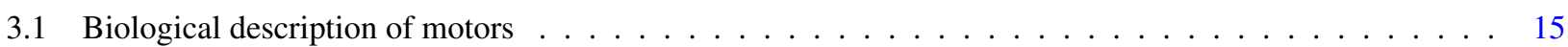

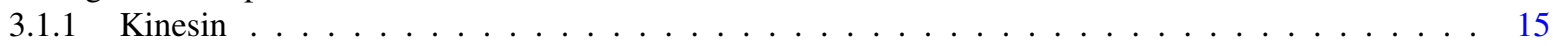

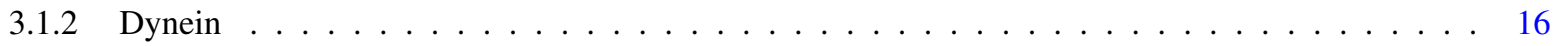

3.1 .3 Kinesin and dynein under load . . . . . . . . . . . . . . . . . . . . 17

3.1 .4 In vitro versus in vivo . . . . . . . . . . . . . . . . . . . . . . . . . . . . . . 18

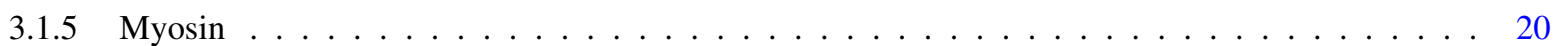

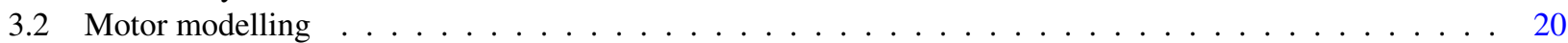

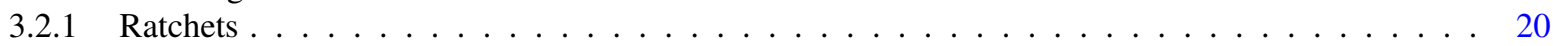

3.2.2 Mechanochemical cycle of molecular motors . . . . . . . . . . . . . . . . 20

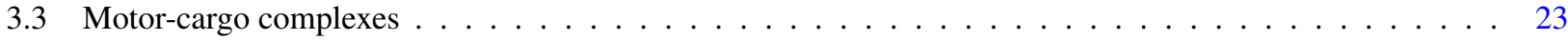

3.3 .1 Transport of lipid droplets . . . . . . . . . . . . . . . . . . . . . . . . 24

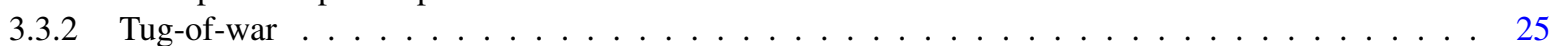

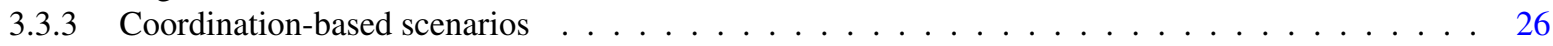

3.3 .4 Transport of large cargos . . . . . . . . . . . . . . . . . . . . . . 26

3.4 Dynamics of molecular motors on filament networks . . . . . . . . . . . . . . . 27

4 Exclusion Processes $\quad 28$

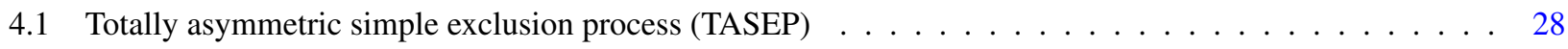

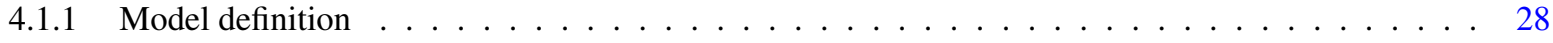

4.1 .2 Update scheme . . . . . . . . . . . . . . . . . . . . . . . . . 29

4.2 Theoretical results for the TASEP with random sequential update . . . . . . . . . . . . . . 31

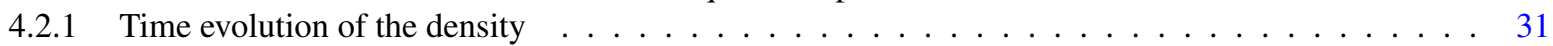

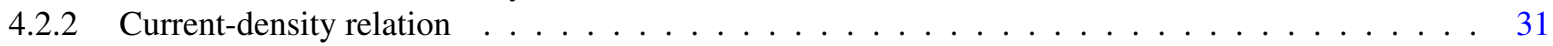

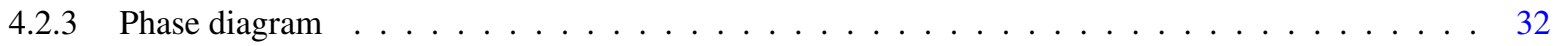

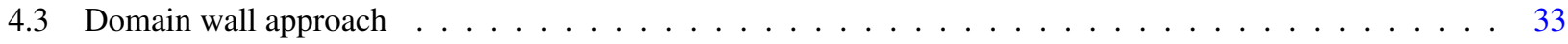

5 Variants of TASEP

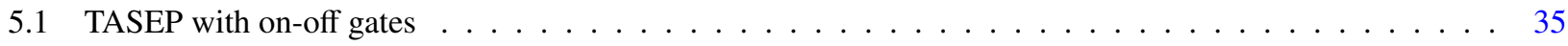

5.1 .1 Single lane TASEP with dynamical gate . . . . . . . . . . . . . . . . . . . . . . . . . . . . . . . . . . .

5.1 .2 Alternating flows in multi-lane TASEPs . . . . . . . . . . . . . . . . . . 36

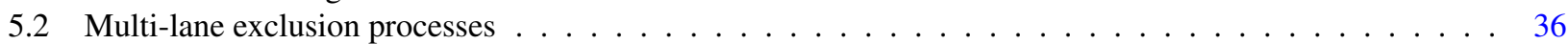

5.2.1 Multi-lane interactions without lane changes . . . . . . . . . . . . . . 36

5.2.2 Multi-lane uni-directional transport with bulk lane changes . . . . . . . . . . . . . . . . 37

5.2.3 Multi-lane bi-directional transport with bulk lane changes . . . . . . . . . . . . . . . . 40

5.3 Modelling inhomogeneities . . . . . . . . . . . . . . . . . . . . . . 40 
6.1 The TASEP with Finite Resources ． . . . . . . . . . . . . . . . . . . . . . . . . . . . . 41

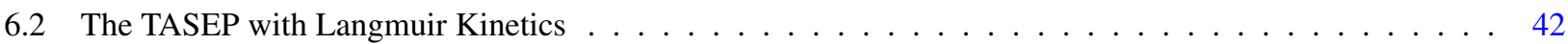

6.3 Explicit Realizations of Particle Reservoirs . . . . . . . . . . . . . . . . . . . . . . . 44

6.4 Enhanced diffusion on filament networks . . . . . . . . . . . . . . . . . . . . . . 46

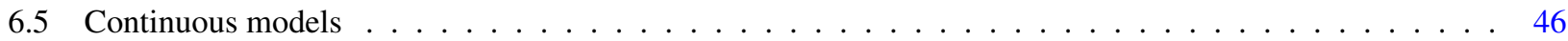

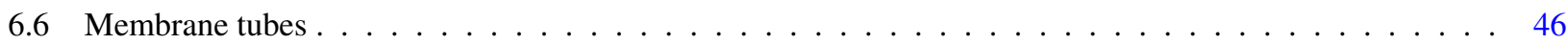

7 Bi-directional transport and spatial confinement 47

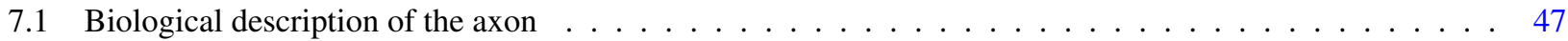

7.1.1 Structure of the axonal microtubule network . . . . . . . . . . . . . . . . . . . 48

7.1.2 Dynamics of the axonal microtubule network . . . . . . . . . . . . . . . . . . . 48

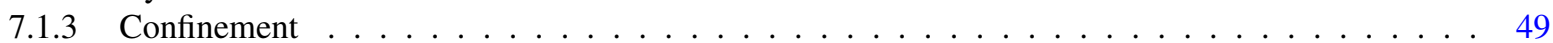

7.2 Finite diffusion: a two-species TASEP coupled to a diffusive lane . . . . . . . . . . . . . . . . 50

7.2 .1 Model definition . . . . . . . . . . . . . . . . . . . . . . . 50

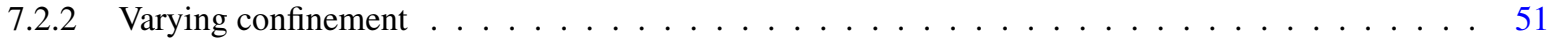

7.3 Lattice dynamics . . . . . . . . . . . . . . . . . . . . . . . . . . . 52

7.3.1 Idealized MT dynamics: model definition . . . . . . . . . . . . . . . . . . . 52

7.3.2 Phase transition to a homogenous state . . . . . . . . . . . . . . . . 53

7.3 .3 Mean-field approximation . . . . . . . . . . . . . . . . . . . . . . 54

7.3.4 Rubustness against different lattice dynamics . . . . . . . . . . . . . . . . . . . . . . 54

7.3.5 Toward more realistic MT network and dynamics . . . . . . . . . . . . . . . . . 55

7.4 Motor-motor interactions . . . . . . . . . . . . . . . . . . . . . 55

7.4 Experimental observations . . . . . . . . . . . . . . . . . . . . . . 55

7.4 .2 Modelling modified attachment rate . . . . . . . . . . . . . . . . . . . 56

7.4 .3 Modelling side-stepping . . . . . . . . . . . . . . . . . . . . 57

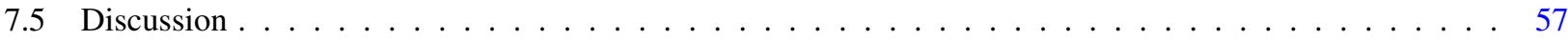

8 Challenges for the development of transport oriented models 5

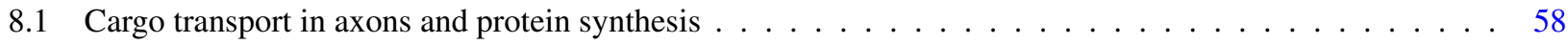

8.2 The transport network: microtubule dynamics in axons . . . . . . . . . . . . . . . . . 59

8.3 Motors action on MTs . . . . . . . . . . . . . . . . . . . . . . . . . . . 60

8.4 Modification of MTs by MAPs : The example of tau proteins . . . . . . . . . . . . . . . . . . . 60

8.5 Structural defects of the MT network . . . . . . . . . . . . . . . . . . . . . . 61

8.6 Global view of model/experiments interplay . . . . . . . . . . . . . . . . . . 62

9 Conclusion $\quad 63$ 


\section{Acronyms}

$\begin{array}{ll}\text { ADP } & \text { Adenosine diphosphate } \\ \text { ASEP } & \text { Asymmetric simple exclusion process } \\ \text { ATP } & \text { Adenosine triphosphate } \\ \text { CLIP } & \text { Cytoplasmic linker protein } \\ \text { DNA } & \text { Deoxyribonucleic acid } \\ \text { DW } & \text { Domain wall } \\ \text { EB1 } & \text { End-binding protein 1 } \\ \text { EE } & \text { Early endosomes } \\ \text { GDP } & \text { Guanosine diphosphate } \\ \text { GFP } & \text { Green fluorescent protein } \\ \text { GTP } & \text { Guanosine triphosphate } \\ \gamma \text { TuRC } & \gamma \text {-tubulin-containing ring complex } \\ \text { HD } & \text { High Density phase } \\ \text { LD } & \text { Low Density phase } \\ \text { MAP } & \text { Microtubule-associated protein } \\ \text { MC } & \text { Maximal Current } \\ \text { mRNA } & \text { Messenger ribonucleic acid } \\ \text { MT } & \text { Microtubule } \\ \text { MTOC } & \text { Microtubule-organizing center } \\ \text { SEP } & \text { Symmetric simple exclusion process } \\ \text { TASEP } & \text { Totally asymmetric simple exclusion process } \\ + \text { TIP } & \text { Microtubule plus-end tracking protein }\end{array}$




\section{Introduction}

The cell is a perfect example of a naturally arisen complex system. In the course of evolution, a large number of biochemical processes assembled forming the smallest of any living organisms' subunits. As evidenced by the immense diversity of life forms on earth, there exist more than one possible combination of these processes fulfilling the basic criteria for life such as reproduction and the existence of a metabolism [249]. The metabolism is the sum of processes which maintain the cell out of equilibrium or - to put it in the words of Erwin Schrödinger [330] - "It feeds on negative entropy." In fact, reproduction involves the transmission of the genetic information stored in the cell's DNA and as is well known from thermodynamics, information transmission is impossible in thermal equilibrium implying that free energy must constantly be imported into the cell. Using the energy directly or indirectly derived from solar radiation to keep the cell in a non-equilibrium state therefore prevents an organism from dying.

The sizes of cells are typically in the micrometer range with eukaryotic cells being larger than prokaryotic ones. The larger size of eukaryotes comes along with a specialization of the various regions of the cell. The spatially distant organelles have to be connected in order to create the complete metabolic chain [10]. This explains the presence of a cytoskeleton in almost all eukaryotes. The cytoplasm being rather crowded, diffusion turns out to be a highly inefficient medium for transportation. Instead, active intracellular transport is necessary. Molecular motors walk along filaments of the cytoskeleton while carrying intracellular cargo of various types from the site of supply to the site of demand [328]. This type of intracellular transport is crucial for the cell's survival and makes the spatial organization possible in the first place. In this review, we are particularly interested in axonal transport. Axons have a quasi-one-dimensional structure with a dense microtubule network, which is a challenging environment for bidirectional motor driven transport.

Different diseases in multicellular organisms, amongst them human neuronal diseases, can be attributed to the failure of intracellular transport [329]. Understanding how the transport works is thus a necessary precondition for understanding why it fails. Statistical physics can be a relevant tool to tackle these questions. Indeed, intracellular transport is a strongly out-of-equilibrium system, where permanent currents take place, and thus meets the increasing interest for understanding how a thermodynamics of non-equilibrium systems could be built. Besides, intracellular transport involves a great number of interactions, some of which still unknown, prohibiting a detailed modeling. Eventually fluctuations play an important role in the dynamics of the various components of the cell. Statistical physics provides tools that allow one to deal with aggregated information and with influential fluctuations, hence rendering it particularly suited for the analysis of the aforementioned complex systems. It already proved to be helpful identifying the elements most crucial for the system's behavior, as we will illustrate in this review.

In this review, we shall first present the actors involved in microtubule transport. We shall start with the "roads", namely microtubules and actin filaments (section 2), and continue with the "vehicles" by presenting the most common molecular motors (section 3). In both chapters, a survey of the biological background and of some modelling attempts will be given.

As exclusion processes will turn out to be useful tools to understand collective effects, their definition and main properties will be summerized in section 4 . Some variants that progressively include features relevant for intracellular transport (multi-lane traffic, static or dynamical defects) will be introduced in section 5.

Langmuir kinetics, i.e. the ability of the motors to attach and detach from the filaments, will be considered in section 6 . The models considering only uni-directional transport are not relevant for intracellular transport but still can describe some in vitro experiments called motility assays, which usually involve only one type of motors. Indeed, some models based on exclusion processes were successful in explaining some jamming phenomena observed experimentally in such motility assays. Another characteristics of these in vitro experiments is that motors that detach from the filament can freely diffuse in the surrounding solution. Confinement around the microtubule becomes an important feature when in vivo transport is considered. Section 6 shows how general classes of boundary conditions and/or geometries of the surrounding diffusion reservoir can modify the transport properties in exclusion processes models.

In section 7 we shall concentrate on axonal transport, which is of special interest as its failure plays a role in several neuronal diseases [316]. We shall present some modeling attempts to take its specificities into account. In particular, we shall explore the role of microtubule dynamics and motor-motor interactions in the global organization of microtubulebased transport.

We shall see that transport in the cell is far from being understood, and that both experimental and modeling efforts are needed. In Section 8, we shall review some other key ingredients for the efficiency of intracellular transport and mention some of the numerous open questions that still require investigation. 


\section{Cytoskeleton filaments}

The cytoskeleton carries out different fundamental functions in biological cells [10]. The cytoskeleton determines the shape of the cell and its spatial organization, it generates forces and serves as a "road network" for molecular motors. Although the structure of the cytoskeleton is quite complex and highly dynamic only three types of cytoskeletal polymers have been identified: actin filaments, microtubules, and intermediate filaments. We restrict our discussion to actin filaments and MTs since they carry the motor-driven transport on the cytoskeleton.

Microtubules (MTs) are the stiffest cytoskeletal filaments and have the most complex assembly and disassembly dynamics as we will discuss in more detail below. MTs can span distances that are of the same order as the diameter of a cell. This underscores its importance as a track for long distance intracellular transport, which is carried out by molecular motors of the kinesin and dynein family. The microtubule network is structured by microtubule organizing centers (MTOC) from which the MTs are growing outwards. Despite its structural stability the MT-network is reorganizing itself on timescales that are relevant for intracellular transport. Therefore, the structure of the MT-network as well as its dynamics has to be considered for the analysis of motor-driven intracellular transport.

Compared to MTs, no structure equivalent to MTOCs exists for actin filaments. Besides, actin filaments are much more flexible. Despite this, the actin network plays an important role in force generation and cell locomotion. The ability to generate forces on the cell membrane is related to the high density of cross linkers that promote the assembly of e.g. actin filament bundles, and branched networks. Also the actin filaments are very dynamic objects, which for example advance the leading edge of migrating cells.

From a physical perspective the dynamics of the cytoskeleton is a process that exhibits generic non-equilibrium behavior. This property is intimately connected with the ability of biological cells to form complex dynamic structures, as for example in the course of cell division. These processes have been analyzed theoretically by macroscopic models, that describe the cytoskeleton as an active gel (see e.g. [177] for a review). In this section we will not discuss the mechanical properties and the spatial organization of the cytoskeleton but rather focus on the structure and dynamics of single MTs and actin filaments.

\subsection{Microtubules}

The design of microtubules must respond to some a priori contradictory constraints [10]: On the one hand, their structure must resist to thermal fluctuations and be able to sustain mechanical stress. On the other hand, they must be able to adapt rapidly to changes (of shape for example) and to respond to the cell needs in a flexible way. These constraints lead to a quite complex structure of microtubules, which we will discuss in more detail: Microtubules are polymers whose fundamental subunit is a $\alpha / \beta$-tubulin heterodimer of $8 \mathrm{~nm}$ length [10]. Each heterodimer is made of a $\alpha$-tubulin and a $\beta$-tubulin, which are both globular proteins that differ slightly.

The tubulin dimers are able to spontaneously assemble along their long axis into protofilaments (figure $1 A$ ). Usually twelve to fourteen of these protofilaments wrap into a helical cylinder of approximately $25 \mathrm{~nm}$ diameter [8] which is then called microtubule. The helical pitch adds up to $12 \mathrm{~nm}$, resulting in an imperfect wrapping and a distinguishable seam in the surface lattice (figure $1 \mathrm{~B}$ ). Due to the asymmetry of the dimer subunit, MTs are locally polar but also as a whole in the sense that the two ends of the filament are chemically different and can therefore be distinguished. We shall see that this polarity will be an important feature for intracellular transport, as it determines the walking direction of molecular motors. The common convention is to define the filament end that exposes $\beta$-tubulin to be the plus-end of the MT.

Microtubules exhibit a characteristic dynamic behavior, termed dynamic instability, characterized by a stochastic switching between phases of persistent growth (= polymerization) or fast shrinkage (= depolymerization) [254]. A transition from growth to shrinkage is called catastrophe and a transition from shrinkage to growth is called rescue.

In vitro, early experiments indicated that both transition rates are independent of the time spent in the respective states [380]. Furthermore, the speed of depolymerization does not depend on the concentration of tubulin in solution, whereas the speed of polymerization on the other hand depends linearly on the amount of tubulin [380]. The rescue and catastrophe frequencies in vivo are usually rather low such that MTs exhibit persistent growth and shortening on the typical length scales of the cell diameter [208]. Therefore, the interaction between MT and the cell boundary is strongly influencing the MT dynamics [110].

Growth at the plus-end occurs in form of an open sheet structure to which single tubulin dimers or small oligomers are added (figure $1 \mathrm{C}$ ). Shrinking MTs have single protofilaments curling outward which peel off the filament and eventually detach from it. A highly idealized view of MT dynamics can be seen in the animated cartoon [374]. 
a

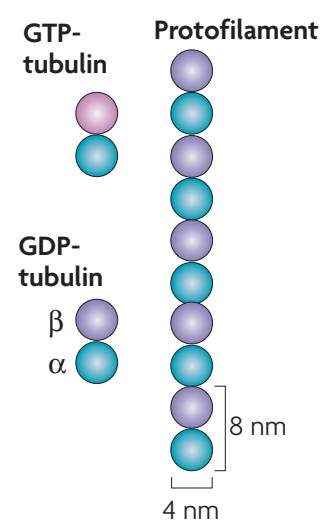

b Plus end

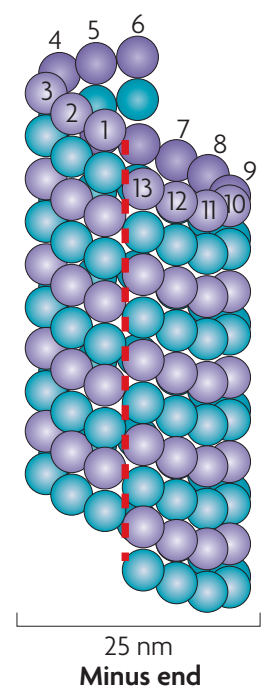

c

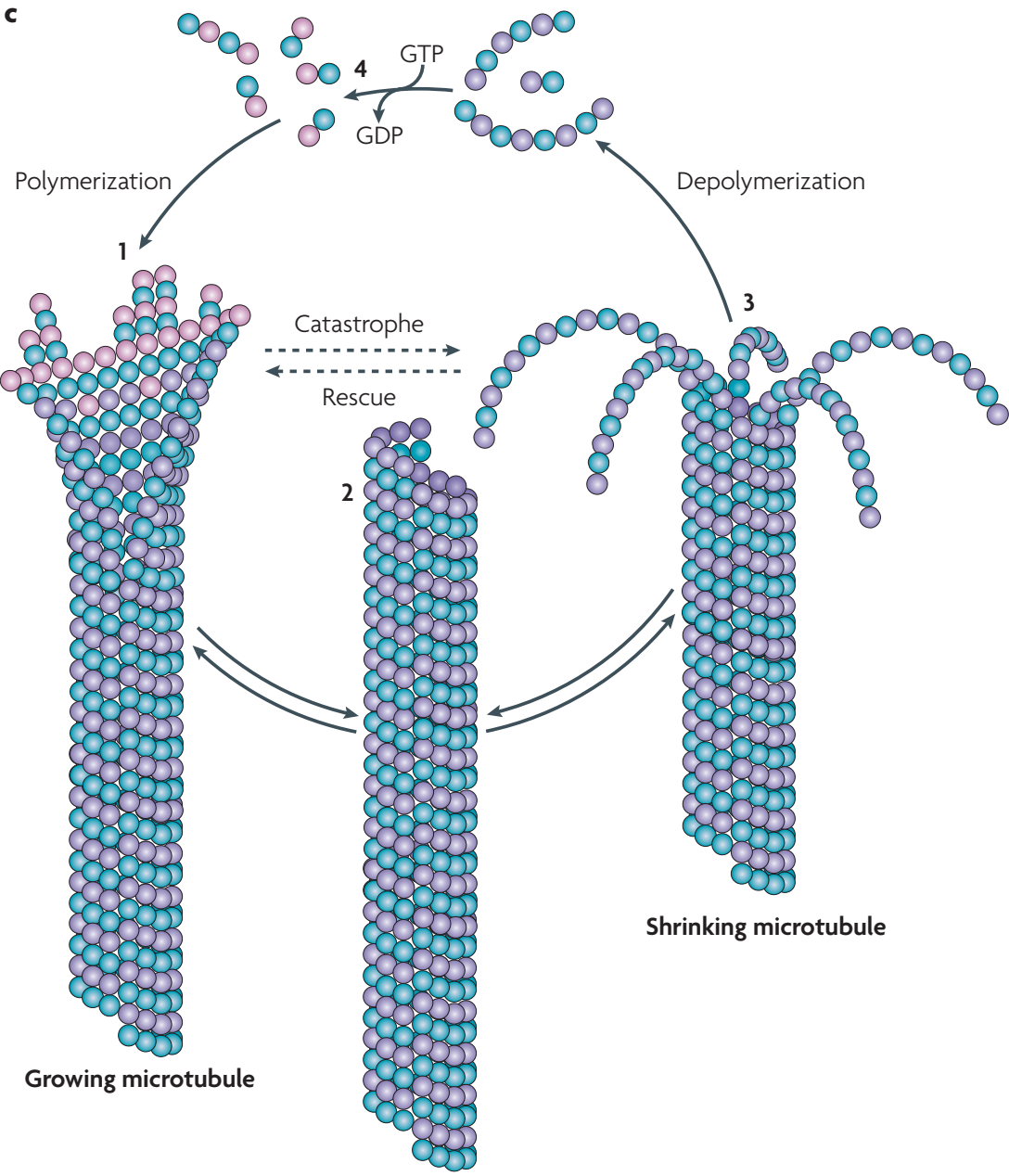

Metastable intermediate microtubule

Figure 1: Microtubule structure and dynamic instability. (A) The $\alpha / \beta$-tubulin heterodimers, either associated to GTP or GDP, assemble into protofilaments of $8 \mathrm{~nm}$ periodicity. (B) About thirteen protofilament helically wrap into a hollow cylinder. The helical pitch is $12 \mathrm{~nm}$ and therefore longer than the periodicity of the MT lattice in axial direction, resulting in a seam (red dashed line). (C) MTs switch between phases of growth and shrinkage. A transition from the polymerizing to the depolymerizing state is called catastrophe whereas the inverse transition is called rescue. 1. In the growing state, the plus-end is in the form of an open sheet with GTP-bound tubulin at its end. At further growth, the MT closes along the seam and the GTP in the bulk of the lattice hydrolyzes to GDP. 2. The existence of a metastable state is possible in which the MT is completely closed and pauses. 3. The protofilaments have lost their lateral contacts at the plus-ends and peel off. 4. Tubulin dimers or small oligomers have their GDP replaced by GTP in solution and are ready to be reincorporated into another growing MT. From [8]. 
The plus- and minus-ends of microtubules obey different kinetics [379]. The plus-end is much more dynamic that the minus-end. Besides, on average, a minus-end, if not anchored, is depolymerizing while a plus-end is polymerizing. In most in vivo systems, the minus-end of the MT is attached to a microtubule-organizing center (MTOC) also called centrosome, inhibiting any dynamics at the minus-end. Then all the dynamical activity occurs at the plus-end.

In vitro, it is possible to observe MTs that are not anchored. As a result of the different kinetics at the plus- and minus-ends, one may observe a very special dynamic regime of the MTs (which is actually much more often observed for actin filaments) called treadmilling [208]: A treadmilling filament polymerizes at its one end while it depolymerizes at the other, thus moving in one direction as a whole filament while every single subunit is immobile. Though this is not the general case, there are also some in vivo systems for which MTs are not anchored and for which treadmilling can be observed. It was the case for fish melanophores fragments [309] and for CHO and NRK cultured cell lines [208].

In order to understand further the dynamical instability of MTs, it is necessary to mention the crucial role played in this process by some nucleotides, the GTP (guanosine triphosphate) and GDP (guanosine diphosphate). Free tubulin dimers are included into the MT with GTP molecules bound to both the $\alpha$ - and $\beta$-tubulin. After polymerization, the GTP bound to the $\beta$-tubulin hydrolyzes to GDP [84]. Therefore one observes a so-called GTP cap at the growing plus-end of the microtubule, while further along the microtubule, most $\beta$-tubulins carry a GDP molecule. Still, 'islands' of GTP can be found inside polymerized MTs in vivo [95]. The relatively long lifetimes of these islands indicate that the stochastic process of GTP hydrolysis occurs on different time scales depending on whether the associated tubulin subunit is exposed at the plus-end or whether it is buried in the MT lattice.

It turns out that GDP-tubulin subunits depolymerize about 100 times faster than GTP subunits [10]. The presence of a GTP cap stabilizes the plus-end of the microtubule and stops rapidly most starting depolymerization events [254]. However, if by chance this protecting GTP cap disappears, the remaining part of the microtubule can depolymerize very rapidly: it is the aforementioned catastrophe. Depolymerization may end when an island of GTP (a GTP remnant inside the MT) is reached [95], inducing rescue, and a new episode of growth.

From a general point of view, it is clear that the unstable nature of MT dynamics makes it very sensitive to external factors and allows the cell to fine tune it as needed. Apart from the role of the GTP/GDP nucleotides, MT dynamics is influenced by a wide range of microtubule-associated proteins . ${ }^{1}$

So far we focussed mainly on the (de-)polymerization of existing microtubules. However, as microtubules may completely depolymerize there is a need to also nucleate new microtubules. Microtubules are able to assemble spontaneously in vitro in the presence of soluble tubulin and GTP [190]. However, nucleation occurs on time scales larger than the subsequent polymerization. It is thus the limiting step in the formation of new microtubules [10].

Controlling nucleation allows the cell to decide where and when MTs will grow. In vivo, some proteins may favor nucleation at specific locations: In many eucaryotic cells, the minus-ends of MTs are therefore located at a MTOC, called centrosome [57,93]. A third tubulin subspecies, $\gamma$-tubulin, was shown to form a $\gamma$-tubulin-containing ring complex ( $\gamma$ TuRC) which is highly implicated in MT nucleation [278, 277, 400]. This complex is formed by approximately 13 $\gamma$-tubulin subunits which form a ring on the centrosome that has almost the same diameter as a MT. The ring is not completely closed but exhibits a helical pitch, which is also recovered in the polymerized MT structure. Hundreds of $\gamma$ TuRC are found on centrosomes in vivo where they cap the minus-end of single MTs which nucleated from these ring complexes. They thus prevent the addition or loss of tubulin subunits at the minus-end of nucleated MTs [400, 260].

\subsection{Regulation of the microtubule dynamics}

A large number of proteins interact with the MT and are consequently classed in the broad family of microtubuleassociated proteins (MAPs). These MAPs are very diverse in structure and function and interact very differently with MTs. While some modify the dynamics of MTs [327], others do not exert an influence on the MTs but rather need the MT lattice to carry out their function. An exhaustive review of MAPs is far beyond the scope of this review. Here, we shall only mention a few of them in order to illustrate typical functions of MAPs which influence the microtubule dynamics.

Certain MAPs, commonly referred to as +TIPs, are able to track the (growing) plus-ends of MTs. A non-negligible part of the research interest in +TIPs arises due to the fact that these proteins are highly conserved over large evolutionary distances and because of the question how the members of this protein class are able to find the end of the MT [335, 132, $66,261,7,8]$. Several mechanisms for plus-end tracking have been attributed to different +TIPs as for example [8]:

\footnotetext{
${ }^{1}$ Some post-translational modifications occuring on tubulin also play a role in specializing the microtubules into a given function. We refer the reader interested in this last point to a review on the subject [167].
} 
1. A + TIP might be able to bind to a specific structure at the plus-end of MTs. Since a growing MT has the shape of an open sheet at its end (see figure 1), it is conceivable that interactions with MAPs are different in this part compared to the bulk of the MT lattice where the sheet has already closed.

2. In a very similar way to the previous mechanism, a +TIP might have a high affinity for a feature at the plus-end but be nevertheless able to bind to the bulk of the MT lattice on which the +TIP performs two-dimensional diffusion until it arrives at the plus-end.

3. As we shall see in section 3.1.1, some family (kinesin) of molecular motors move along the MT in the direction of the plus-end of the MT. These motors can thus carry +TIPs to the plus-end.

4. +TIPs might be added to the MT through a co-polymerization mechanism, i.e., tubulin dimers or oligomers associate in solution with the +TIP before these tubulin subunits are added to the filament.

5. Some +TIPs might themselves not be able to find the plus-end but bind to another + TIP which is able to do so, a scenario which is termed hitchhiking.

Specific + TIPs have been found for each of these mechanisms and consequently, no single mechanism for plus-end tracking can be identified. The recognition of a specific structure at the plus-end and the co-polymerization mechanism (scenarios 1 and 3) have in common that they lead to treadmilling of +TIPs: The +TIPs themselves do not move but are added at the tip and then released deeper down in the MT lattice without performing lateral motion.

One prominent example is the cytoplasmic linker protein-170 (CLIP-170) which has been shown to increase the rescue frequency of MTs in vivo and therefore positively influence the average lifetime of MTs [206]. The binding process of CLIP-170 is rather complex and details can be found in [207, 38, 37, 99, 96]. Here, we just notice that CLIP-170 temporarily binds to the plus-end of MTs and is released with a given rate.

Therefore both, the GTP-cap as well as the +TIPs have a strong influence on the microtubule dynamics and act in a qualitatively similar way. The key mechanism is that GTP as well as +TIPs are introduced at the plus-end of growing microtubles and reduce significantly the rates of catastrophes. The stabilizing molecules are only temporarily bound to microtubles. Either they unbind from the filament with a given rate, as for example the +TIPs or they lose their stabilizing function, as in case of the GTP-hydrolysis. This means that fast growing microtubules are rather stable, since they are stabilized by a cap, which increases the rescue rates or decreases the catastrophe frequencies. By contrast, microtubules that are slowly growing or pausing at the cell membrane become gradually more unstable.

Several in vitro and in vivo experiments have been carried out, that analyze the consequences of the aforementioned stabilizing mechanisms. In [101, 380] it was shown in vitro that the catastrophe frequency decreases with increasing polymerization speed, which was controlled by the GTP-concentration. The stabilization also leads to length and lifetime distributions which differ from those one would observe for constant polymerization/depolymerization rates [134]. The impact of different stabilization mechanisms has been studied in vivo. Some length distributions were found experimentally to be exponentially confined near the cell edge [208]. However, it was also shown that these distributions were only obtained if some cytoplasmic linker proteins (CLIP) were present [206]. Indeed, in the absence of CLIP, the microtubule rescue was found to be reduced by sevenfold, with the consequence that MTs were often completely depolymerizing. The resulting length distribution was then found to be flat [206] instead of exponential.

\subsection{Modeling the dynamical instability: the two-state model}

In this section, we discuss one of the early models introduced by Dogterom and Leibler [98] describing the dynamics of MTs with a fixed minus-end. In this model, the MT can either be in the growing ("+") or shrinking ("-") state. The MTdynamics are characterized by four rates: the growth rate $v_{\mathrm{g}}$, the shortening rate $v_{\mathrm{s}}$, the rescue rate $v_{\mathrm{r}}$ and the catastrophe rate $v_{\mathrm{c}}$. This set of parameters is sometimes extended by a fifth parameter $c$ which gives the concentration of free tubulin and may be used as argument of some of the other rates. The MT is treated as a one-dimensional chain of $l$ subunits to which subunits can be added and removed at the plus-end.

Assuming a constant concentration of free tubulin, the concentration $c$ can be ignored in this simple model and the master equation for the probabilities to observe a filament of length $l$ in the growing or shrinking state far from any 
boundary is given by $[98]^{2}$ :

$$
\begin{aligned}
& \frac{\partial p_{+}(l, t)}{\partial t}=v_{g} p_{+}(l-1, t)-v_{g} p_{+}(l, t)-v_{c} p_{+}(l, t)+v_{r} p_{-}(l, t) \\
& \frac{\partial p_{-}(l, t)}{\partial t}=v_{s} p_{-}(l+1, t)-v_{s} p_{-}(l, t)+v_{c} p_{+}(l, t)-v_{r} p_{-}(l, t) .
\end{aligned}
$$

In this representation, the plus sign in the index denotes the growing state and the minus sign the shrinking state. Space is discretized in a natural way as MTs possess with the tubulin dimer a well-defined structural unit. This model presents a phase transition between bounded and unbounded growth with the transition taking place at

$$
v_{\mathrm{s}} v_{\mathrm{c}}=v_{\mathrm{g}} v_{\mathrm{r}} .
$$

The MT length distribution in the bounded growth phase is exponentially decaying with mean

$$
\langle l\rangle \approx \frac{v_{\mathrm{g}} v_{\mathrm{s}}}{v_{\mathrm{s}} v_{\mathrm{c}}-v_{\mathrm{g}} v_{\mathrm{r}}}
$$

while in the unbounded growth regime, the average length grows linear over time as

$$
\langle l\rangle=\frac{v_{\mathrm{g}} \nu_{\mathrm{r}}-v_{\mathrm{s}} v_{\mathrm{c}}}{v_{\mathrm{c}}+v_{\mathrm{r}}} t .
$$

Above, it was assumed that the free tubulin concentration $c$ was homogeneous, i.e. that free tubulin was diffusing very fast compared to the time scales of MT dynamics, and time independent.

Including spatially and temporally varying concentration $c$ of free tubulin dimers, which are supposed to have a finite diffusion constant, dynamical states can be created in which two populations of MTs exist simultaneously: While one population exhibits unbounded growth, a second one is trapped at finite MT lengths because of a lack of free tubulin which has been consumed by the first population [98]. This result thus shows that the environment influences MT polymerization and may play a role in the regulation of the cytoskeleton.

An alternative way proposed in [170] to account for the finite supply of free tubulin is to consider that the MT dynamics takes place in a small volume. The authors assume that the free tubulin concentration is homogeneous (fast diffusion), but can be globally modified by the consumption or release of tubulin during polymerization and depolymerization. The fluctuations of tubulin concentration are larger in small volumes. Indeed, at equal initial concentration, the amount of tubulin available in small volumes is more limited and will be more easily depleted by the growth of the MT. As a consequence, the distribution of MTs lengths, which is exponential for large volumes, becomes a gamma-like distribution in the limit of small volumes [170]. Another consequence is that strongly confined MTs would appear more dynamic, which might for example be relevant for the axonal MT network (see section 7.1.2).

In case of filaments that are not connected to a MTOC or to any other anchoring structure, one has to consider the dynamics of the minus-ends in a similar fashion, but with different rates. Free minus-ends allow for the displacements of the filament as a whole, i.e. the treadmilling dynamics which has been previously discussed.

The dynamics of filaments is not only restricted to the plus- and minus-ends if the action of MT-severing proteins such as katanin ${ }^{3}$ is considered. This has been introduced into the model of Dogterom and Leibler in [363] by considering randomly occurring severing events. The MT is then split into two with the newly exposed plus-end being in the shrinking state. This modification could also be shown to lead to a finite average MT length with non-exponential length distributions. Interestingly, the severing does not increase the total number of MTs although the average MT length decreases.

\subsection{Models with explicit description of the GTP cap}

In the previous discussion of the MT dynamics we underscored the importance of the GTP cap for the MT-dynamics, which significantly changes the length distribution.

\footnotetext{
${ }^{2}$ Actually, the equations in [98] were written in a space continuous form.

${ }^{3}$ Katanin forms a hexamer around the MT and uses the energy from ATP hydrolysis to break the filament [298]
} 
Therefore, a different class of MT models has been suggested, which does not postulate switching between a growing and a shrinking state, but describes the process at a more refined scale [126, 247, 12, 60]. In these models, individual tubulin subunits are allowed at all times to be added to or removed from the plus-end. Subunits which are added always carry a GTP and have low (or vanishing) probability to depolymerize from the plus-end. The GTP then hydrolyzes to GDP at a certain rate after the subunit has polymerized. The hydrolysis event modifies the subunit's kinetics : A subunit bound to GDP (instead of GTP) has a higher probability of being removed when it is exposed at the plus-end.

A consequence of these evolution rules is that the density of GTP bound subunits is highest at fast growing plus-ends. This class of models is thus adequately reproducing the presence of the GTP cap mentioned in section 2.1. The GTP cap has an average length which is determined by the rate of hydrolysis of GTP to GDP and by the attachment rate of new tubulin subunits. Indeed, as GTP is hydrolyzed, its density is decreasing with time, such that the concentration of GTP is low far from the plus-end or in the case of slowly growing or pausing filaments.

These models are thus microscopic in the sense that there is no state variable describing the filament as a whole as with the growing and shortening state of the two-state model. Nevertheless, these models exhibit a macroscopically observable behavior which reproduces the long periods of persistent growth and shrinkage characteristic for the dynamic instability of MTs [126, 247, 12, 60]. The rescue and catastrophe rates assumed in section 2.3 are in these models observables which result from the kinetics of the tubulin subunits with GDP or GTP. For example, in [247] the rate of catastrophes observed in the model scales as $n^{-2}$ if $n$ is the average length of the GTP cap. As for the two-state model of section 2.3, considering a fixed concentration of tubulin in the absence of geometrical constraints can lead to bound or unbound growth of the MTs depending on the tubulin concentration [247, 12].

There are still several other features that may have to be taken into account for a full picture of the dynamic instability. For example a dependence of the catastrophe rate on the age of the microtubule observed in vitro [133] still has to be explained. Some recent molecular dynamics simulations of the microtubule [149] may help to distinguish between the various scenarios that have been proposed. While only spontaneous hydrolysis of the GTP was considered here, other scenarios including induced hydrolysis (i.e. hydrolysis favored if neighboring GTPs are already hydrolyzed) were explored in [126] and shown to explain some dilution experiments.

The loss of the GTP cap is not the only factor that could induce a catastrophe. Structural changes at the tip, interactions between protofilaments, or MAPs, could also play a role [60]. Comparing models and experiments in various situations could help to distinguish between these different scenarios, as we will illustrate below by the example of dynamic MTs in finite volume.

\subsection{Dynamic MTs in finite volume}

The interaction between a growing MT and the cell boundary is of great importance, since catastrophes are induced with a very high rate when the MTs reach the cell boundary.

The simplest way to explicitly consider the cell boundary is by imposing a maximum filament length $l^{*}$ such that $l \leq l^{*}$ as in [142], where a two-state model has been considered. In this work it is assumed that the MT directly turns into the shrinking state, if the MT hits the cell boundary. For these boundary conditions the size of the MTs is always limited, irrespective of the model parameters. The stationary state length distribution can be calculated exactly and is given by $p_{ \pm}(l) \sim a^{l}$, where $a=\left(1+v_{r} / v_{s}\right) /\left(1+v_{c} / v_{g}\right)$. Therefore, the length distribution is exponentially growing if $v_{g} v_{r}>v_{s} v_{c}$ (corresponding to unbounded growth in the open system) and exponentially decaying if $v_{g} v_{r}<v_{s} v_{c}$. In the case of very small rescue and catastrophe rates, rather flat length distributions are obtained, corresponding to typical life-history plots as those of Fig. 3 B \& D.

Similar results were obtained with models describing explicitely the GTP cap [144]. Note that we only consider the case of a sufficiently high concentration of tubulin, so that the growth of MTs is not limited before the cell edge is reached [144].

The two regimes can be obtained experimentally : Length distributions exponentially confined near the cell edge were indeed found experimentally [208]. However, it was also shown that these distributions were only obtained if some cytoplasmic linker proteins (CLIP) were present [206]. Indeed, in the absence of CLIP, the microtubule rescue was found to be reduced by sevenfold, with the consequence that MTs were often completely depolymerizing. The resulting length distribution was then found to be flat [206] instead of exponential.

The effect of such a +TIP that enhances rescue - and thus stabilizes MTs - was modeled in [110]. A summary of the model dynamics is given in Fig. 2. The key feature of this model is the following: When a tubulin dimer is added, it is assumed to present a binding site to which rescue enhancing +TIPs bind at very high frequency (taken to be infinite here). 


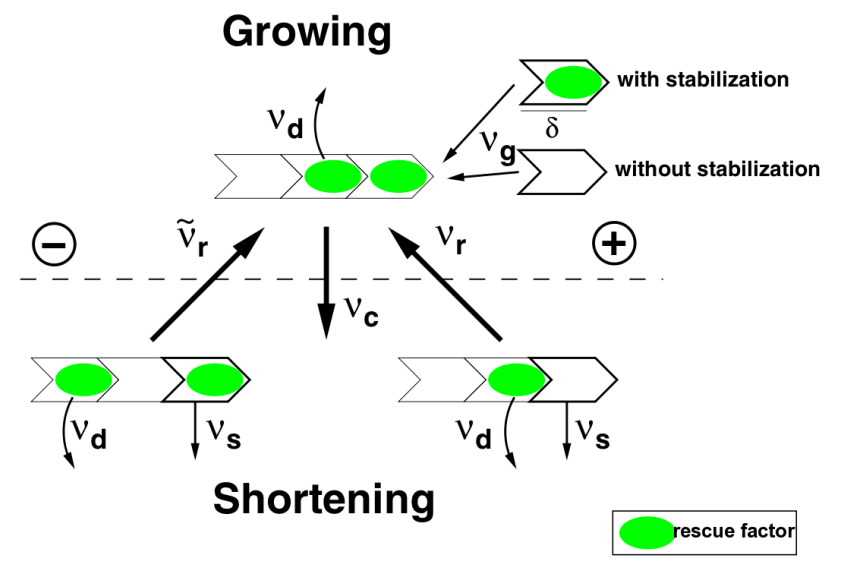

Figure 2: Definition of a model for filament dynamics. The filament can be either in a growing state or in a shortening state. When it is in the growing state, a subunit of length $\delta$ is added at a rate $v_{\mathrm{g}}$ to its plus-end. Depending on the scenario, the added subunit carries a rescue-enhancing + TIP or not. The filament will switch to the shortening state with rate $v_{\mathrm{c}}$. Then the filament loses subunits from its plus-end at rate $v_{\mathrm{s}}$ regardless of the possible presence of a rescue factor. However, the rate for switching back to the growing state (rescue) depends if a rescue factor is present on the last subunit at the plus tip (then the rate is $\tilde{v}_{\mathrm{r}}$ ) or not (then the rate is $v_{\mathrm{r}}<\tilde{v}_{\mathrm{r}}$ ). At any time, a tubulin subunit can loose its rescue factor at rate $v_{\mathrm{d}}$. Figure modified from [110].

When the last tubulin subunit at the +tip carries a rescue factor, it modifies accordingly the rescue rate, which increases then from $v_{\mathrm{r}}$ to $\tilde{v}_{\mathrm{r}}$. A tubulin subunit included in the MT can loose its rescue factor with rate $v_{\mathrm{d}}$. This model will be compared to a reference case without rescue factor (without stabilization in Fig. 2).

Interesting effects are observed if the model filament is embedded into a finite predefined geometry mimicking the shape of a cell within which the origin of nucleating MTs is chosen as described in the caption of Fig. 3. A MT grows in a random direction from the MTOC. The maximum distance $l^{*}$ it can grow before reaching the boundary depends on this direction. The filament follows the bulk dynamics as described above until it reaches the boundary. Then no further tubulin subunit can be added. At the boundary, switching to the shrinking state is induced with a probability $p_{\text {ind }}$.

Fig. 3 compares some life histories obtained from the model of Fig. 2 [110], and from the experiments [206], with or without rescue factor. The model reproduces well the observed behavior, namely the large length fluctuations in the absence of CLIP170 (as almost no rescue will prevent the filament to entirely depolymerize), and the pauses and frequent switching between growth and shortening near the cell edge when CLIP170 is present. Also typical lifetimes have the right order of magnitude. Note that in the model, it is assumed that the +TIP is only enhancing rescue. One could think of +TIPs that would rather prevent catastrophe to start. However, this would not explain the experimentally observed behavior, as it would not lead to the numerous rescues (see Fig. 3C).

Interestingly, the model predicts an aging effect in the survival probability distribution of the MTs. Indeed, the survival probability of an MT after it hits the cell boundary for the N-th time is found to be lower for higher values of N [110]. This aging effect, which has also been discussed later in $[133,134,6]$ from an experimental point of view, is not observed in the model if no rescue factor is included. This aging effect is of great importance since stabilized MTs show a great resistance to thermal fluctuations and mechanical constraints. But at the same time the MTs can completely depolymerize after the stabilizing cap is removed from the filament. This way MTs are able to fulfill the contradictory constraints with respect to their rigidity and flexibility.

\subsection{Actin filaments}

Actin filaments are composed of only two twisted protofilaments. As a result, they are much more flexible than MTs and their radius is only 5-9nm [10]. However, they form highly interconnected lattices and as a whole have strong mechanical properties. Indeed, they play a major role to give to the cell membrane its shape and mechanical strength.

In the cell, actin filaments are usually embedded into a network, and submitted by myosin motors to non-thermal fluctuations. The mechanical properties of such an acto-myosin gel can be modeled by macroscopic non-linear models which describe the cytoskeleton as a whole [212, 236]. A review of these approaches can be found in [177]. Some microscopic stochastic models have also been developped (see for example [225, 224]). Some acto-myosin active gels 
A
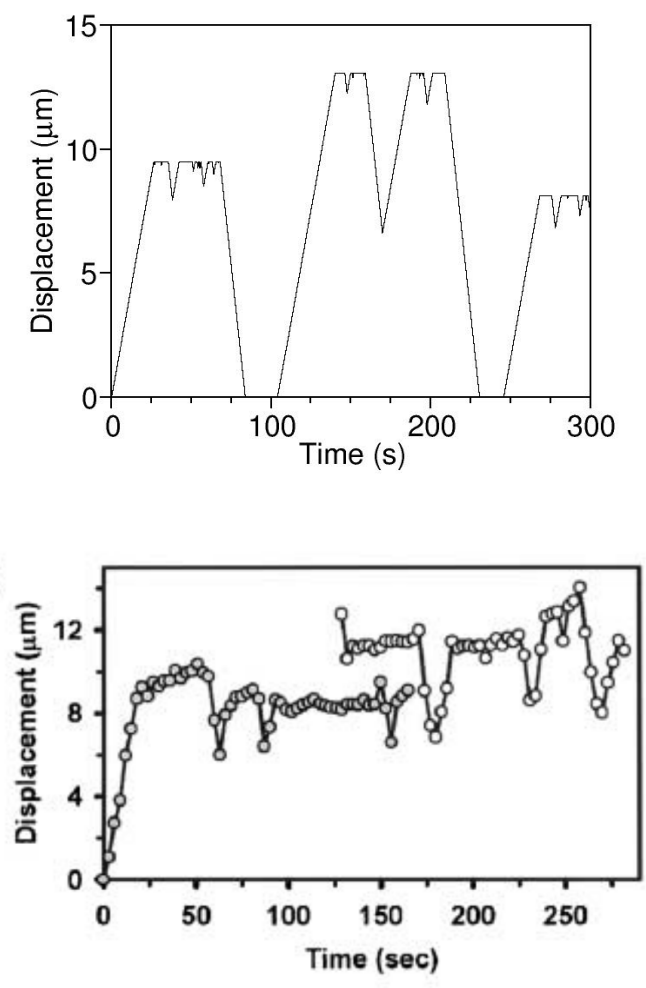

B
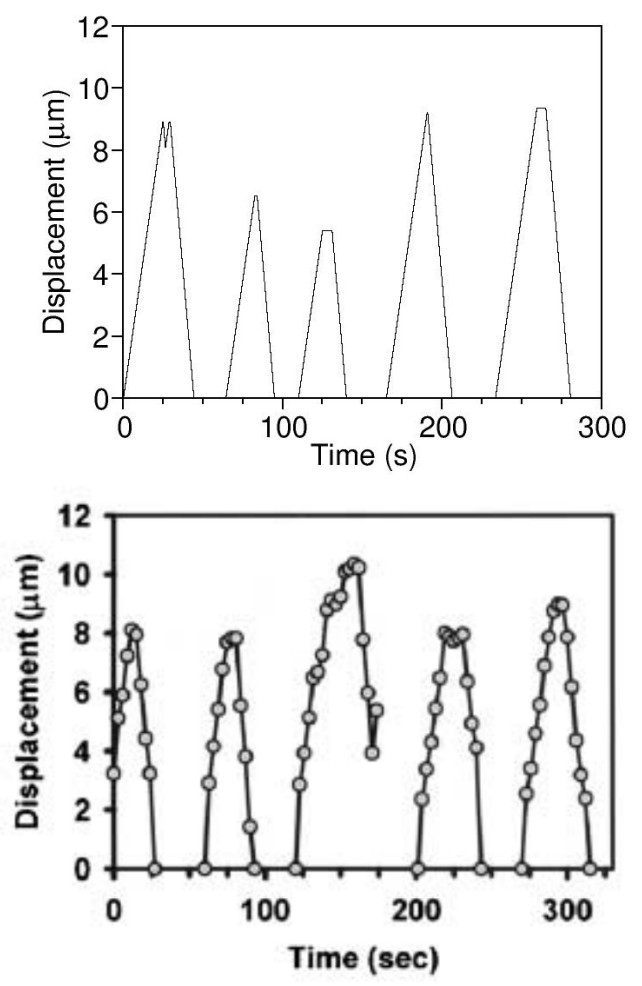

$\mathrm{D}$

Figure 3: Life history plots of $(A-B)$ simulated or $(C-D)$ real MTs $(A-C)$ with and $(B-D)$ without rescue-enhancing +TIPs. If tubulin subunits carrying a rescue factor are added under growth $(A-C)$, the MTs show dynamic instability at the cell boundary with frequent rescues. Without rescue-enhancing +TIPs $(B-D)$, the MTs depolymerize completely after a short contact with the cell boundary, and MT lifetimes are mostly determined by the time needed for polymerization up to the cell boundary and depolymerization back to the MTOC. The geometry for the simulations was an ellipse of half-axes $a=19.2 \mu \mathrm{m}, b=6.6 \mu \mathrm{m}$ with the MTOC being $1.2 \mu \mathrm{m}$ off the center of the ellipse in $x$ - and in $y$ - direction which are representative parameters for the cell geometry discussed in [206]. Figures $(A-B)$ (simulations) are taken from [110] and figures $(C$ - $D)$ (experiments) from [206]C2002 (doi:10.1083/jcb.200208058). 


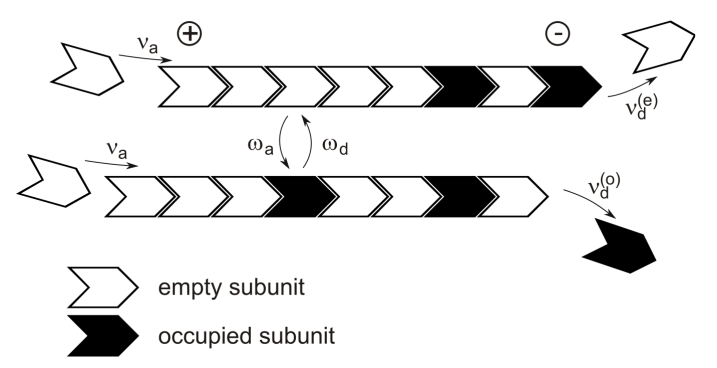

Figure 4: Schematic representation of a treadmilling filament. Subunits are added at the plus-end with rate $v_{a}$. A protein can bind to an empty subunit with rate $\omega_{a}$ and detach with rate $\omega_{d}$. A subunit is removed at the minus-end with rate $v_{d}^{(e)}$ if it does not carry a protein and with rate $v_{d}^{(o)}$ if it does. From [114].

have been studied in in vitro experiments [52, 203, 11, 104]. We shall not address this large field of research in this review. Instead, in this section we rather concentrate on the dynamics of single filaments.

Like MTs, actin filaments are polar polymers, i.e. one can distinguish a fast growing plus-end and a minus-end where the filament disassembles rapidly if it is not stabilized. Actin filaments are dynamical structures, evolving through mechanisms similar to MTs. The GTP/GDP nucleotides embedded in MTs are replaced by ATP/ADP that play a similar role in the case of actin. As actin filaments are not attached to a centrosome, they naturally undergo treadmilling both in vitro and in vivo.

An important difference between MT and actin filaments is that the dynamics of actin filaments combines both polymerization and depolymerization events on short time scales, which can be averaged on mesoscopic time scales to give effective growth and shortening rates while the MTs stay, as previously discussed, on much longer periods of time in one - polymerizing or depolymerizing - state.

If we mention actin filaments in this review, this is not because of their mechanical tasks - a subject which is out of our scope - but because they also serve for intracellular transport. We shall see that a specific family of molecular motors uses the actin filaments as tracks (see section 3.1.5), and though we shall not develop the role played by actin lattices in intracellular transport, it should be kept in mind that, as a complement to MT based transport, part of the transport is performed along the actin networks [23] that, for example, cover the inner side of the cell membranes. This is in particular true for transport along the axon (section 7.1).

\subsection{Treadmilling filaments}

Actin filament dynamics can be simply described by an average growth rate at the plus-end and an average shrinking rate at the minus-end. A stochastic model that realizes this dynamics is the one of Fig. 4 (in the case where only empty subunits are considered). The filament is represented as a one-dimensional chain of finite length, to which subunits are added with rate $v_{a}$ at the plus-end and removed with rate $v_{d}$ at the minus-end. This simple description can also apply to MTs that are not anchored (as possible for example in in vitro experiments) when the dynamics is considered on time scales large enough to average over the large fluctuations due to the dynamical instability.

Unregulated polymer assembly typically leads to exponential length distributions, with a length scale that is given as a function of polymerization and depolymerization rates. The length distributions of unregulated polymers is rather broad. This is not what is observed in certain situations, for example for the actin filaments that are found in stereocilia in the inner ear where length must be well controlled [317]. Therefore, several mechanism have been suggested that influence the polymerization dynamics. For example, a simple mechanism of length regulation has been introduced in [114]. In this work the decoration of tubulin subunits with a protein that can alter the depolymerization rate at the minus-end is assumed (see Fig. 4). Depending on the (de-)polymerization rates and the concentration of the length-regulating protein, one observes different dynamical regimes, i.e. unbounded filament growth, exponential distributions and unimodal distributions, which can be very sharp. The unimodal distributions are observed in case of proteins that enhance the depolymerization rate. In this case it is possible to reduce the length fluctuations significantly, since the relative fraction of occupied subunits is increasing with the filament length, and thereby increasing the effective depolymerization velocity for relatively long filaments. 


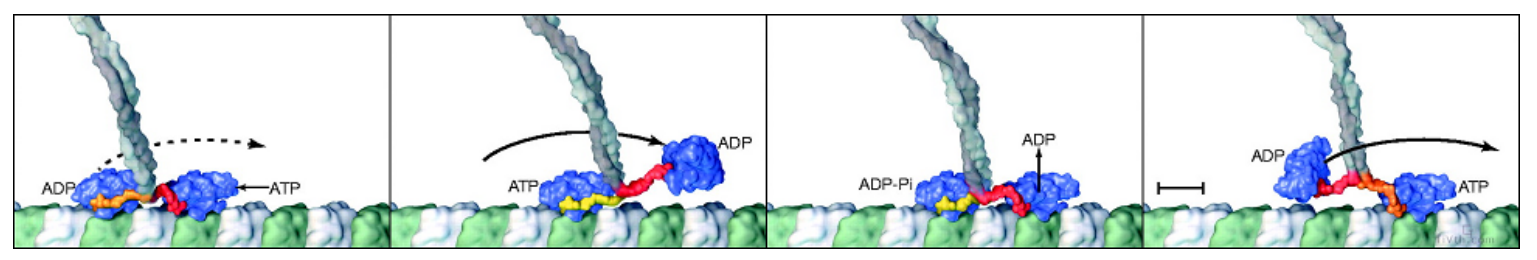

Figure 5: Stepping cycle of kinesin. From left to right: An ATP-bound head induces a conformational change which brings the ADP-bound head to the front. Then the ATP of the now rear head gets hydrolyzed to ADP and inorganic phosphate. After the release of the inorganic phosphate, the rear head unbinds from the filament and is brought to the front as soon as the other head exchanges its ADP by ATP. From [370].

\section{Molecular motors}

While previous section was devoted to the "roads" along which intracellular transport occurs, this section will be dedicated to the "trucks", i.e., the molecular motors that drag intracellular cargos such as organelles and vesicles along the cytoskeletal filaments.

\subsection{Biological description of motors}

Cytoskeletal motors - also known as motor proteins - are molecules which are able to move along polar cytoskeletal filaments such as MTs or actin filaments by using the energy derived from hydrolysis of adenosine triphosphate (ATP): they are active particles. Hydrolysis of ATP induces a confirmational change of the motor protein that results into a step along the filament.

Motor proteins can detach and attach to the filaments. An important characteristics of motors is the number of steps or conformation changes - that they can handle in a row before detaching. A so-called processive motor is able to perform a large number of steps before detaching, while a non-processive motor will usually detach after a single step. Examples of non-processive motors are myosins II, which are responsible for muscle contraction and have to work as a team to exert a continuous force. By contrast, MT-based transport involves processive motors which, individually, are able to interact for long period of times with the track and to move over large distances [329].

These motor proteins usually consist of at least two parts: the motor domain ${ }^{4}$ and the cargo-binding domain. The motor domain is able to bind to the polar filament, while the cargo domain binds to intracellular cargos such as organelles or other elements of the cytoskeleton [10, 243].

In the following, the two most important protein families for MT-based transport are presented, kinesins and dyneins [369]. Both families are heterogeneous in the sense that the cargo which they are able to carry as well as the type of motion along the filament varies within the same family despite similar structures of the motor domains [160]. In order to restrict our presentation, we shall focus on the most important representatives of each family for MT-based transport, namely kinesin-1 and cytoplasmic dynein. We shall also briefly address the case of some myosins able to carry cargos along actin filaments.

\subsubsection{Kinesin}

The kinesin superfamily is subdivided into several tens of kinesin families [307] which exhibit surprisingly similar motor domains and therefore are all able to exert force on MTs [371]. Due to the remaining differences in the structure of the molecule, motion patterns vary: While most of the kinesins move toward the plus-end of a MT (anterograde movement), some kinesins move in the opposite or retrograde direction. Also, many but not all kinesins perform processive motion.

In this review, we shall focus on kinesin-1 (also called conventional kinesin), which is a representative member of the kinesin superfamily implicated in long-range transport and which moves toward the plus-end of MTs. Note that in the remaining of this review, we shall use the work "kinesin" to refer to kinesin-I, unless otherwise stated.

Kinesin- 1 is composed of two heads ${ }^{5}$ that can bound to the MT, linked by a long neck to the cargo domain (Fig. 5). The two motor heads move in a coordinated manner, and step in an alternating hand-over-hand pattern on the MT [370].

\footnotetext{
${ }^{4}$ The motor domain refers to the part of the protein which is responsible for force production

${ }^{5}$ Note that head refers to what would be called a foot if kinesin was considered as a walker.
} 


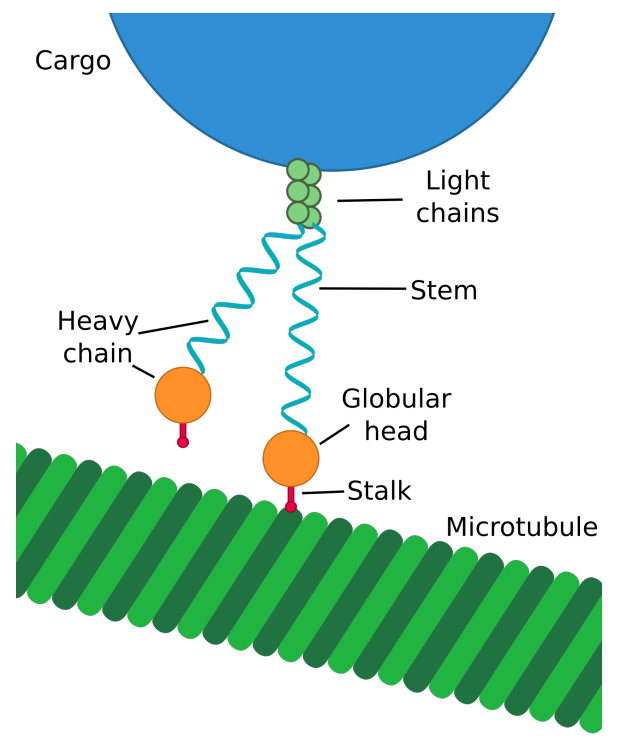

Figure 6: A stylized depiction of dynein carrying cargo along a microtubule.

The stepping is triggered by the hydrolysis of ATP [83] (figure 5): An ATP-bound kinesin head has a high affinity to the MT lattice and binds strongly to it. When the ATP is hydrolyzed into adenosine diphosphate (ADP) and inorganic phosphate, the binding of that head gets weaker and the motor head eventually detaches from the filament. Meanwhile, the other motor head was able to take up an ATP and bind to the MT, inducing a conformational change that brings the recently detached motor head to the front. As soon as this motor head replaces its ADP by ATP and re-attaches to the filament, the cycle starts over again. The step size of kinesin, which is half of the step size of each motor head [394], has been shown to be equal to one tubulin subunit, i.e., $8 \mathrm{~nm} \mathrm{[394,} \mathrm{123].}$

Several of these stepping cycles occur before kinesin detaches from the MT. The length of the displacement performed along the MT by a molecular motor between two phases of diffusion in the cytosol is called its run length. Typical run lengths for kinesin are of the order of one hundred steps [337] which in combination with the step size of $8 \mathrm{~nm}$ gives runs of about $1 \mu \mathrm{m}$ in length. Kinesin is thus a processive motor.

It is in general considered that conventional kinesin has a uni-directional motion. Indeed, without load, back-stepping is exceptional for kinesins: less than $1 \%$ of the net distance covered by a single kinesin corresponds to backward stepping [244]. However, if kinesin is attached to a cargo, its stepping behavior depends on the force (or load) exerted by the cargo onto the motor. Back-stepping becomes non negligible under high backward load [42, 41]. Backwards steps even predominate above stall force [65]. This leads obviously to a dependence of the velocity with the force exerted on the motor.

An applied force does not only affect the stepping but also the detachment rate of kinesin. We shall discuss in more details the velocity-force and detachment-force relations in section 3.1.3.

\subsubsection{Dynein}

The dynein family is composed of two groups: axonemal dyneins, involved in the motion of flagella or cilia, and cytoplasmic dyneins, which play an important role in intracellular transport. Only the latter group will be considered here.

Cytoplasmic dynein carries out most of the minus-end directed long-range transport along MTs. The attachment to the wide variety of different cargos is probably assured by different multifunctional adaptors such as dynactin which regulate dynein function [187]. The structure of cytoplasmic dynein is more complicated than those of kinesins - a fact which is also reflected in its very high molecular weight of 1.2 MDa [256]. Indeed, among molecular motors, dyneins are the largest one [10]. Each dynein head has the shape of a ring, with a stalk that stretches out from the head and binds to the MT. The cargo is attached at the end of a long tail pointing away from the MT in the bound state. 
Dynein, in the absence of nucleotide, is bound to the MT and it is when an ATP binds that it can detach. This is in contrast with kinesin, which associates to the MT when an ATP is bound, and is released through the hydrolysis of ATP [10]. However, it was found in some experiments that dyneins may step forward and backward under load even in the absence of nucleotide hydrolysis [136].

Dynein is able to perform processive motion if it is in dimerized form [383]. The run lengths of the processive motion are of the order of $1 \mu \mathrm{m}$ and thus comparable to those of kinesin [383, 302].

Contradictory results have been reported about the step size of single dyneins. In [242], it was reported that under no load, dyneins were taking predominantly 24- and 32-nm long steps, and that the step length was decreasing under load. Later, more direct measurements of dynein displacement were performed through high temporal resolution fluorescence. While some authors found that most steps of dyneins were $8 \mathrm{~nm}$ long, whatever the load is [364], others report a variability in the step size larger than for kinesins [136]. Actually, results depend on whether it is the head or tail of dynein that is marked [302]. While there is a peak of the step size distribution around $16 \mathrm{~nm}$ for the heads and 8nm for the tail, distributions are much larger than in the case of kinesin, and exhibit a non-negligible fraction of backwards steps [302, 136]. Actually about $20 \%$ of the net distance moved by dynein is backward [244, 302].

Recent measurements [299] have revealed that the nature of dynein stepping depends on the distance between its two heads: Stepping is stochastic and uncoordinated for heads close to each other, but becomes coordinated when the distance between heads is large (indeed then tension forces between the heads tend to bring forward the head most behind). It is still an open question to understand how the uncoordinated stepping of close heads does not alter processivity, as it should lead to higher simultaneous detachment of both heads. The load exerted by large cargos may contribute to coordinate stepping, an hypothesis that has still to be explored [299].

By contrast with kinesin, dynein performs easily side-steps to adjacent proto-filaments when walking on a MT [383, 384, 313, 302].

As a general conclusion, it appears that single dyneins have a much less regular motion than kinesins and can move faster. The varying step size and the side steps have been hypothesized to allow the motor to bypass obstacles [69, 299]. Due to its irregular stepping properties, dynein has sometimes been compared to a drunken sailor [62], while kinesin would be a serious guy, walking straight and regularly.

The binding site of dynein on the MT surface overlaps with the binding site of kinesin [257] (though motor heads of dynein are much larger than those of kinesin [10]). Both motor species thus compete for binding sites on the filament and probably block each other on encounters in bidirectional transport on MTs. We shall study these collective effects later in the review.

\subsubsection{Kinesin and dynein under load}

The processivity of a molecular motor along a MT depends on its binding, stepping, and unbinding rates, which determine the typical run length of the motor on the filament. These rates depend on several external factors, and in particular (for the stepping and detachment rate) on the load exerted on the motor.

In this section, we shall focus on the velocity-force and detachment rate-force relations, which have been measured experimentally. Typically, a coated bead is attached to the motor [357, 166]. The bead is placed in an optical trap, while the motor steps on a MT (Fig. 7). If the optical trap is immobile, the walking motor pulls on the bead until the force exerted by the trap prevents it to move further. Then the motor stops, and the stall force (defined as the force that has to be applied in order to stop a motor) can be calculated from the displacement of the bead inside the trap. In another type of experiment, the optical trap is moved in such a way that the applied force is constant. The velocity-force relation can then be directly measured. The detachment rate can also be measured in these experiments as a fonction of the load.

The stall force under load for kinesin was measured to be around $5-7 \mathrm{pN}$ [204, 376, 244]. Contradictory results have been found for the stall force of dynein. Most experiments indicate a smaller stall force than for kinesins [244, 215]. For example from in vitro data, Kunwar et al [215] estimated $F_{s}=4.7 \pm 0.04 \mathrm{pN}$ for kinesin and $F_{s}=1.36 \pm 0.02 \mathrm{pN}$ for dynein. However in a few experiments stall forces similar to those of kinesins (approximately 7pN) were reported [136]. It has been sometimes mentionned that in vivo values could differ from their in vitro counterparts [215]. However, it is much more difficult to control for example the number of motors involved in an in vivo measurement.

Though there is no general agreement on the precise form of the dependence of velocity or detachment rate with the load, we give here for illustration the expressions used in particular in [215]. 


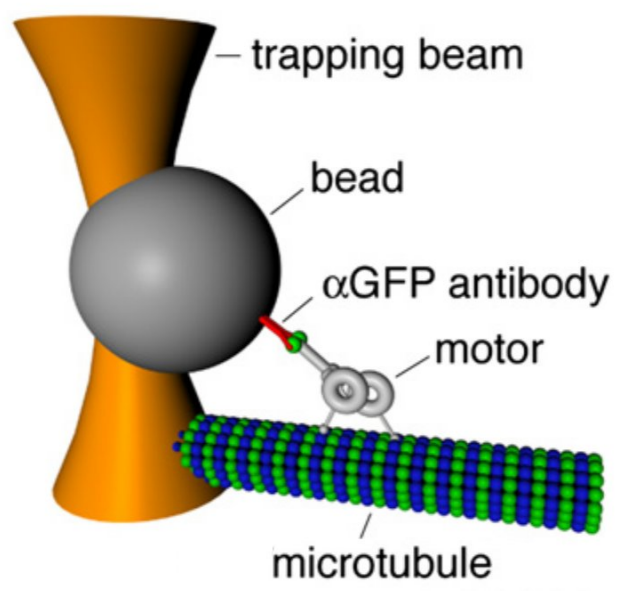

Figure 7: Sketch of an experiment to explore the stepping of a motor under load. Modified from [136].

In [356] the following velocity-force relations of single motors have been suggested:

$$
v(F)=v_{0}\left(1-\left(\frac{F}{F_{s}}\right)^{w}\right) .
$$

where $F$ denotes the amplitude of the force applied to the motor and $F_{s}$ the so-called stall force, which is defined as the force for which the velocity of the motor vanishes. The choice $w=1$ corresponding to a linear force-velocity law is a good approximation (see the experimental results of Fig. 8) and has been used for example in the model of [199]. In [215], Kunwar et al rather took $w=2$ for kinesin and $w=1 / 2$ for dynein.

The force-dissociation relations differ below and above stall. Kunwar et al [215] propose to write the detachment rate as $\epsilon=\epsilon_{0} \Omega(F)$ where $\epsilon_{0}$ is the detachment rate without load (typically between 0.25 and $1 s^{-1}$ ) and $\Omega(F)$ gives the variation with the load, different for kinesins and dyneins. Fits of in vitro data yield [215]

$$
\Omega(F)=\left\{\begin{array}{ll}
\exp \left(\frac{F}{F_{d}}\right) & \text { for } F<F_{s} \\
1.07+0.186 \frac{F}{f} & \text { for } F \geq F_{s}
\end{array} \quad\right. \text { for kinesin }
$$

and

$$
\Omega(F)=\left\{\begin{array}{ll}
\exp \left(\frac{F}{F_{d}}\right) & \text { for } F<F_{s} \\
\left(0.254\left[1-\exp \left(-\frac{F}{1.97 f}\right)\right]\right)^{-1} & \text { for } F \geq F_{s}
\end{array} \quad\right. \text { for dynein. }
$$

where $F_{d}$ is the characteristic detachment force $(4.01 \pm 0.07 \mathrm{pN}$ for kinesin, $0.87 \pm 0.04 \mathrm{pN}$ for dynein), and $f=1 p N$ determines the force scale. These curves are represented in Fig. 9.

\subsubsection{In vitro versus in vivo}

The discussion of the experimental results on the dynamics of molecular motors has shown that remarkable differences between in vitro and in vivo characteristics exist [332]. Some authors [215] report for example that stall forces are completely different in vivo, and in particular that they become similar for dyneins and kinesins [223] (which have quite different stall forces in vitro). The origin of these differences are so far not understood. It is not clear why motors should behave differently in the cell or in vitro, and differences could possibly by artefacts from the interpretation of measurements which are much more difficult to do in a controlled way in vivo. However, it is also possible that the motor activity is strongly regulated by the cell. The properties of the motors could be changed by the viscoelasticity of the cytoplasm (in a similar way as it has been shown that mechanical properties of MTs can be quite different in a viscoelastic medium [53]). Complementary proteins, as for example the tau protein, exist which can bind to MTs and reduce the 


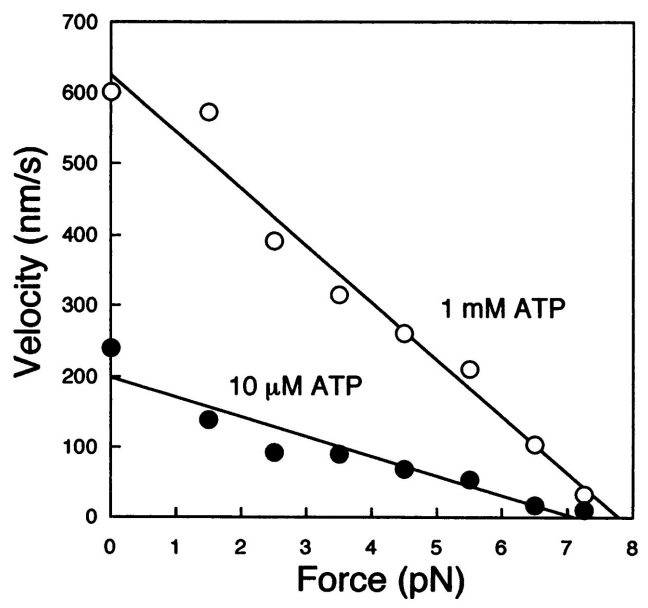

Figure 8: Velocity of kinesin as a function of the backward force, measured in vitro using an optical trap. From [204].
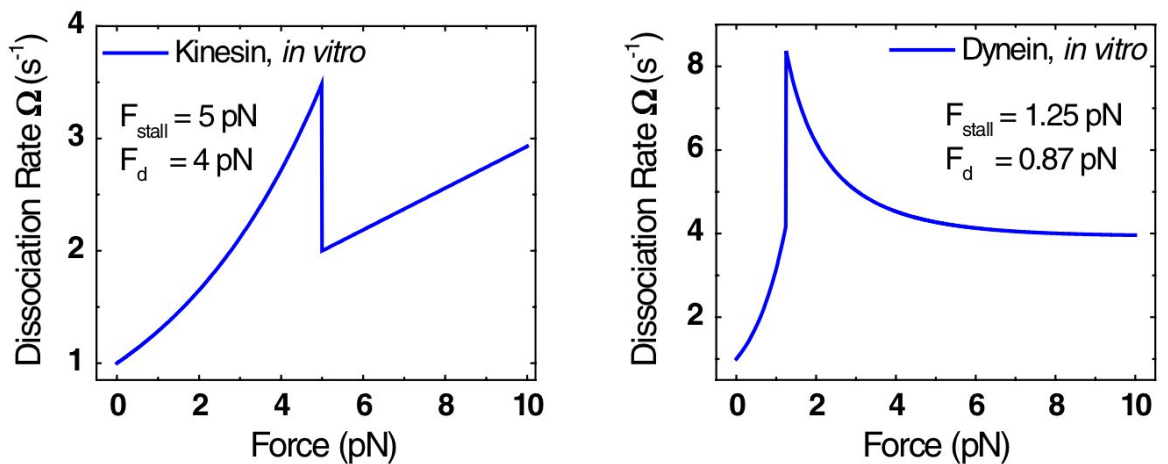

Figure 9: Dissociation rate under load for kinesin (left) and dynein (right). From [215]. 
binding affinity of molecular motors [96]. The attachment rate could also be influenced by regulatory proteins binding directly to the head region of molecular motors [244].

Another important difference between in vivo and in vitro experiments is that the energy supply (i.e. ATP concentration) is not controlled inside cells. Though mitochondria provide most of the energy needed, they are localized, while fast axonal transport requires a constant supply of energy. Some recent experiments [398] with axons have shown that the transported vesicles may themselves carry some glycolytic machinery that provides on-board energy.

\subsubsection{Myosin}

Though we shall not consider it later in the review, we present here briefly the myosin motor family for completeness, as some members of this family are involved in intracellular transport.

Cytoskeletal myosin motors move along actin filaments. The most famous one (called myosin II) is present in muscles. The coordinated motion of a large number of these myosins is responsible for the contraction of muscles. But many other myosins were discovered later, which all move toward the plus-end of actin filaments, except myosin VI that move towards the minus-end [387]. In this review we are interested in myosins involved in actin-based axonal transport. This is the case of myosin $\mathrm{V}$ which transport organelles and vesicles $[358,10]$ and is able to take large steps of $36 \mathrm{~nm}$, which correspond to the actin helix pitch [329]. Similarly to kinesin, it steps through a hand-over-hand mechanism [393].

\subsection{Motor modelling}

Several modelling approaches can be pursued to model cytoskeletal motors [56]. We shall now review a few of them and discuss their domain of applicability.

\subsubsection{Ratchets}

A first family of models is based on ratchets. Motor proteins live at a very small scale, where viscous forces are important and where thermal fluctuations play an important role.

The principle of a ratchet is to bias the effect of the fluctuations in order to produce some work. Its mechanism is illustrated in Fig. 10. Imagine a particle subject to a potential that varies periodically with time [314]. At each time period, an asymmetric potential $U_{\text {on }}$ is first applied during $\tau_{\text {on }}$. Then it is switched off during $\tau_{\text {off }}$ (Fig. 10A). During the on-period, the probability distribution to find the particle at a given location concentrates around the minima of the potential $U_{o n}$ (Fig. 10B). During the off-period, the distribution peaks enlarge into gaussians due to diffusion. When the potential $U_{\text {on }}$ is switched on again, particles located in the hatched part of the distribution will move to the right due to the asymmetry of the potential while the others will remain in the same minima. Of course the various time scales involved in this process have to be tuned appropriately in order to obtain net motion.

Note also that this mechanism is highly out-of-equilibrium; an external input is needed to switch between on and off states. The transition rates between the two states are kept away from the spontaneous values that they would have under Boltzmann equilibrium [297].

The link with motor protein motion can be made in the following way [176]. Motors are considered to have two states, in which they interact differently with the filament. They switch from one state to the other through the conformational change triggered by ATP hydrolysis[24]. In each state, the interaction with the filament can be modelled by a potential which is both periodic in space and asymmetric, due to the periodic and polar structure of the filament. If the potentials in each state are different (different amplitude, or shifted in space), it is possible to obtain net motion [297], even in the absence of diffusion [71].

Such a simple ratchet model can be used to model the individual motion of, for example, kinesin on a microtubule. Ratchet models are also appropriate to describe collective motion of sliding motors, such as myosins teams in muscle contraction [178, 179, 176]. However, for collective MT-based transport, exclusion between the motors play an important role which is not well described by this family of models. We shall see in the next chapter another family of models, the exclusion processes, that naturally includes this characteristics.

\subsubsection{Mechanochemical cycle of molecular motors}

We have seen in the previous section how the switching of motors between different internal states could result into a net motion. While ratchets models are useful to understand how oriented motion is generated, we shall now present a family of models that rather focus on the mechanochemical cycle itself. These models allow in particular to explain the 


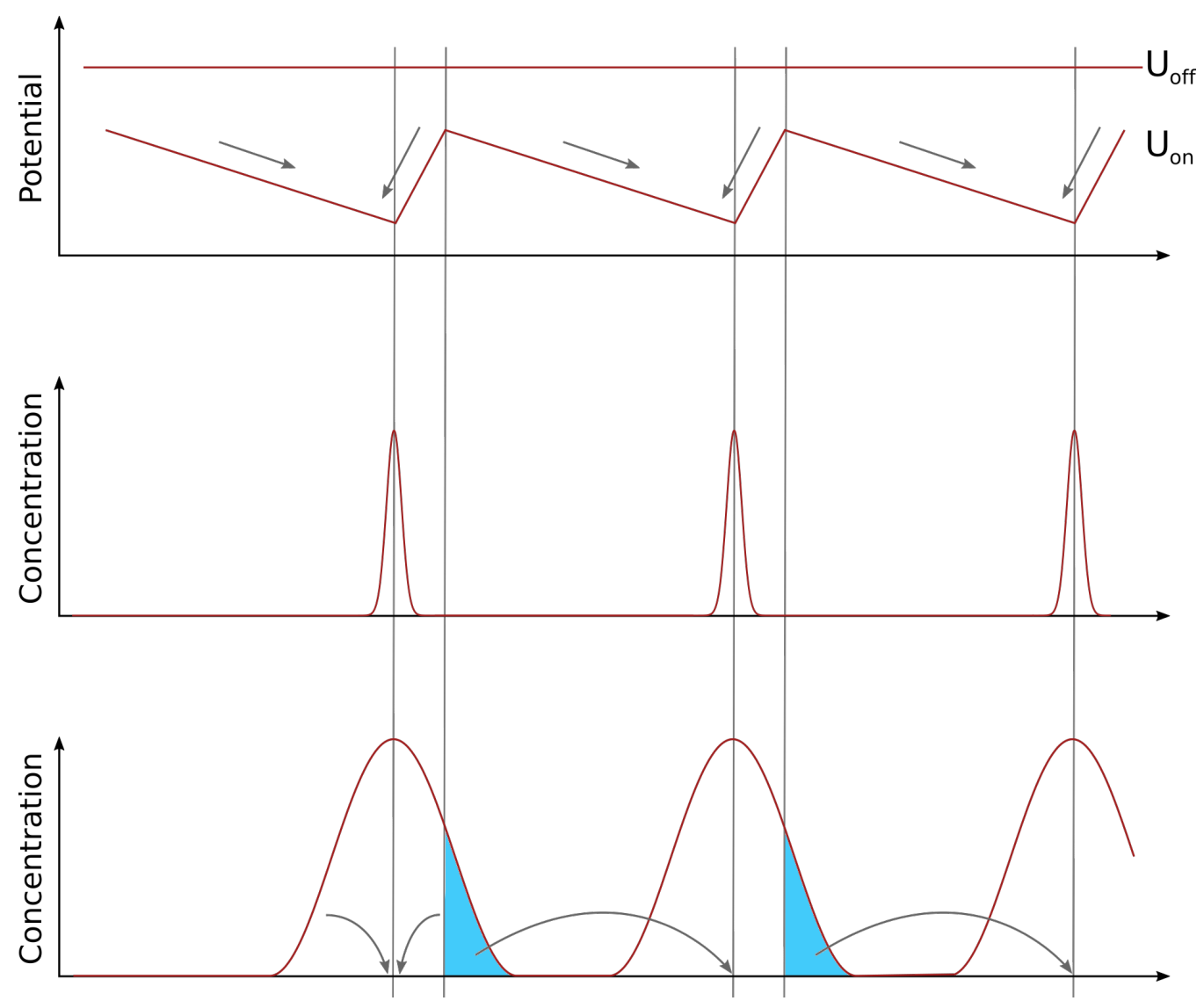

Figure 10: Principle of a ratchet. (A) on- and off-potentials. (B) Distribution of particles after the on-period. (C) Distribution of particles after the off-period. 


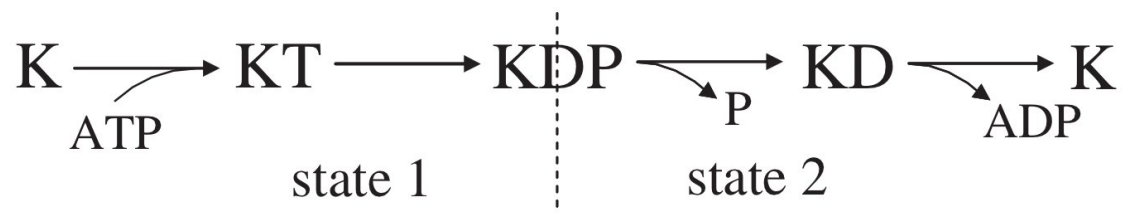

Figure 11: Mechanochemical cycle of kinesin (K). While the kinesin head is attached to the MT in state 1 , it diffuses freely along the MT in state 2. From [276].

variations of the motor stepping rate that are experimentally observed when ATP concentration is varied [221, 222], or when a force is applied to the motor.

One step of a motor is the result of several transitions between different biochemical states. Depending on the degree of detail that is needed, the number of the transitions that are considered may vary [125]. It is commonly accepted that the mechanochemical cycle of a kinesin contains four main transitions [125, 42], as represented in Fig 11: Binding of ATP, hydrolysis of ATP (which produces ADP + inorganic phosphate P), release of phosphate (P), and finally release of ADP. The model can be simplified by merging the states corresponding to a kinesin head strongly bound to the MT (states K and KT in Fig 11 - state KDP has a too short lifetime to be considered) and those where the kinesin head diffuses freely along the MT (state KD). This results into a two-state ${ }^{6}$ model $[181,276,219]$ that already captures important features of the stepping cycle.

The motor displacement is represented in a simplified way, as successive hopping events on a discrete lattice (whose sites would correspond to the potential minima of figure 10A). More precisely, if we take the example of the two-state model, and denote the state of a given lattice site by 0 if the site is empty, or by 1 (resp. 2) if it is occupied by a motor in state 1 (resp. 2), the stepping dynamics along the MT can be described by the following transitions [276]:

$$
\begin{aligned}
& \text { Hydrolysis: } \quad 1 \rightarrow 2 \quad \text { with } \omega_{h} d t \\
& \text { Ratchet: } \quad\left\{\begin{aligned}
2 & \rightarrow 1 & & \text { with } \omega_{s} d t \\
20 & \rightarrow 01 & & \text { with } \omega_{f} d t
\end{aligned}\right. \\
& \text { Brownian motion: } \quad\left\{\begin{array}{l}
20 \rightarrow 02 \\
02 \rightarrow 20
\end{array} \quad \text { with } \omega_{b} d t\right.
\end{aligned}
$$

These rules implement the fact that oriented motion of kinesin via a ratchet mechanism can only take place when kinesin is in state 2 .

The various transition rates may depend on the applied load and on the share of work that is carried out in each substep. Some rates also depend on the ATP concentration. Estimating these contributions allows to calculate a number of experimentally testable quantities, for example the stall force and the load dependent velocity of the molecular motor, or the dependence of velocity on the ATP concentration [125, 181, 219].

Conversely, some informations on the mechanochemical cycle can be extracted from experimental measurements of the variations of stepping rates under various conditions [204]. As these processes are stochastic (and the stochasticity is indeed included in the models), not only the mean value of the motor displacement but also the distribution of the fluctuations can bring some information on the mechanochemical cycle, for example to estimate the number of states in the cycle [42].

A theoretical frame for fluctuation analysis has been provided in [219]. Due to the generic non-equilibrium features of the motor dynamics, generalized fluctuation theorems have to be applied where the Einstein and Onsager relations are violated. It has been shown in this work that the degree by which the Onsager symmetry is broken is determined by the asymmetry of the effective potential which governs the motion of the motors. It turns out that the most efficient dynamics of kinesin is observed if the violation of the Onsager symmetry is maximal.

Using the models of this section, it is not only possible to study the influence of an external force but also the role of a disordered potential landscape [181], which would model the heterogeneity of the intracellular environment. The

${ }^{6}$ This two-state model for motors should not be mixed with the two-state model for filaments of section 2.3. 


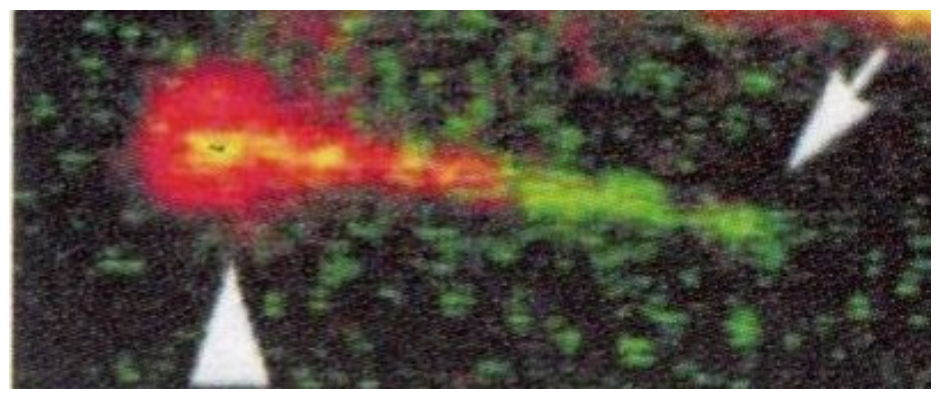

Figure 12: Domain wall in kinesin motility assay. The triangle indicates the plus-end of the MT (green), where kinesins (red) accumulate. The arrow points towards the minus-end. A domain wall separates the high kinesin density region from the low density one. From [275].

resulting dynamics is reminiscent to a Sinai walk [165], where the exponent which describes the velocity dependence on the size of the system, depends on the slope of the potential.

The models of this section describe well the motion of isolated motors, but they can also be extended to many motor systems. Nishinari et al [276] have considered the collective motion of two-state molecular motors. The motility of the motors depends on the concentration of ATP, as explained above. The interaction between motors is implement via an exclusion rule: Only one motor can occupy a given site. It was shown that, when attachment and detachment is included, this two-state model is able to reproduce several features of in vitro experiments, particularly the existence of domain walls between high and low motor density regions [276, 275] (see Fig. 12). This model was extended to several parallel filaments in [75].

An extensive review on the stochastic mechano-chemical kinetics of molecular motors can be found in [74].

We have seen in this section a first example of model where the exclusion between motors is implemented. We shall describe exclusion processes in much more details in chapter 4. For the remaining of this review, we shall not consider anymore the details of the mechanochemical cycle. However, the dependence of the stepping rate on ATP concentration or on load force, which can be explained with the models of this section, will be used whenever needed.

\subsection{Motor-cargo complexes}

Molecular motors transport various types of cargos [388, 112, 237] of different sizes, such as filament precursors, mRNA granules [377], lipid droplets [389], viral capsids [232], lysosomes, or even objects as large as mitochondria [162, 21, 322].

One important fact that has to be considered for a large variety of intracellular transport phenomena is that, in most cases, cargos are not transported by single but by multiple motors which are attached to their surface [21, 154]. Experimental evidence for the attachment of many motors is based on a number of observations, in particular based on force measurements [345, 351, 215, 223]. Another argument is that, for several motor-cargo systems, there is evidence for bidirectional non diffusive motion [388]. Note that the attached motors bind individually to the MT and may a priori have opposite preferential directions. It is thus not obvious to understand how they cooperate to transport a given cargo.

In vitro, simpler systems involving only one type of motors can be studied. For example, some experiments have been carried out where dynein coats beads transported along immobile microtubules [244]. In these experiments it was shown that transport is much more efficient when more than one dynein is attached to the bead. An obvious interpretation is that, when one motor detaches, another one can take on. In this special setting, strongly directed motion is observed, with a typical cargo displacement of the order of several hundreds of micrometers. In [244] evidence was given that the stall force for multiple dyneins seem to be additive.

In vivo, both dynein and kinesin are present and can attach to a given cargo. There is a need to understand in a more refined way how multiple motors handle cargos, for example in order to discriminate between coordinated and uncoordinated motion of the motors. A first hypothesis could have been that only one species of motors would be attached to the cargo at a given time: Motors would just share the load but all of them would be pulling in the same direction (Fig. 13(A)). However, this scenario could be ruled out for several systems [233]. It is rather observed that motors of different types (i.e. having opposite preferred velocities) are attached at the same time to a given cargo [216]. There is a need to understand how the cargo displacement emerges from these multiple interactions. 
Experimentally, one has to distinguish the cargo displacements at short time scale, along a single filament, and displacements over large time scale, during which the cargo may have followed different MTs, oriented in various directions. In the latter case, the randomness of the MT network itself may lead to superdiffusive cargo dynamics [339]. In this section, we shall concentrate on cargo transport along a single filament, while the effect of network will be addressed in section 3.4.

From a practical point of view, it is more easy to study dynamics along single filaments in vitro. However, until recently, it was not known how to handle purified mammalian dynein in motility assays ${ }^{7}$, thus until now, most data on cargo transport by teams of motors have been obtained in vivo. One way to limit the aforementionned network effects in living cells is to consider one-dimensional systems. We shall see in chapter 7 that in axons, the MT network is quasi-1D. But some other systems can also be considered. Epithelial cells, for example, share with axons the property of having a highly polarized MT network. Also, in late Drosophila embryos, lipid droplets are moved on a quasi unipolar MT network [266]. Many studies have been devoted to the transport of lipid droplets, in order to follow typical trajectories of particles that are driven by teams of molecular motors, and we shall report several such experimental results in the next section.

\subsubsection{Transport of lipid droplets}

The most extensively studied cargo transport by teams of molecular motors is the transport of lipid droplets [389], which can also be traced in vivo. In order to characterize the dynamics of lipid droplets several techniques can be used. The most widely used are based on fluorescence microscopy [143]. The high contrast and resolution of this method allows to track individual lipid-droplets very accurately. Besides, it allows to follow many droplets at a time. Despite its obvious advantages there are also some limitations. First of all the observation time (or observation frequency) may be limited by photo-degradation. It has also been observed that the excitating laser is heating the cell and thereby is influencing the dynamics of the observed objects [267].

More recently, various imaging methods have been developped that do not require to introduce an external label in the system. Confocal reflection microscopy [135] measures the light reflected by the particle (e.g. lipid droplet). This technique is less invasive, and allows for higher rate measurements. It is of course restricted to reflecting particles. Differential interference contrast (DIC) microscopy is based on variations in the index of refraction. All these techniques, if used in an optimized way, can achieve an accuracy of the order of 5 nanometers [64]. Other techniques that do not require labels are based on harmonic generation [385] and coherent Stokes Raman scattering (CARS) microscopy [180]. Several techniques can be combined to mark different elements of the cell.

Whatever the technique, almost all experiments report the same type of data: Lipid droplets motion is usually characterized in terms of alternating runs and pauses (note that the precise distinction between runs and pauses is somewhat arbitrary as it depends on the choice of a threshold [284]). Typical data report the length and duration of single runs and pauses. Runs may occur in both directions (though with possibly asymmetric distributions) and can be quite large (up to thousands of nanometers [135]). Pauses could be attributed to several features. Detachment and diffusion periods could be longer than in vitro as they take place in a viscous environment [244]. However, estimations of the number of attached motors (around five of each type in [215], 1 or 2 kinesins against 4 to 8 dyneins in [351]) make the full detachment of the cargo quite unlikely. Competition between different types of motors, or obstacles could also explain these pauses.

It is commonly accepted that the lipid droplets are transported over long distances. But there is no clear experimental evidence whether these large displacements which are found in short-term measurements of uncorrelated large bidirectional runs persist over longer time scales, i.e. whether there is really a net drift of the overall motion. Little is known about the net drift over large time scales. It would be an important information to discriminate between models. Current techniques allow a tracking of lipid droplets over long periods that should give access to this data [135].

Bidirectional motion can be useful even if there is no net displacement (non biased random walk) [378]. Indeed, the random walk behavior of the cargo may be useful just to explore the space, and deliver for example vesicles at the proper place, through a trial and error mechanism [378]. ${ }^{8}$

\footnotetext{
${ }^{7}$ Motility assays are in vitro experiments in which usually one (but possibly more) type of motors performs processive motion along some MTs put in a solution or anchored on a surface.

${ }^{8}$ Alternating slow and fast random motion has been proven as an efficient search strategy ("intermittent search"), which is realized in several biological systems (see [56] and references therein.
} 


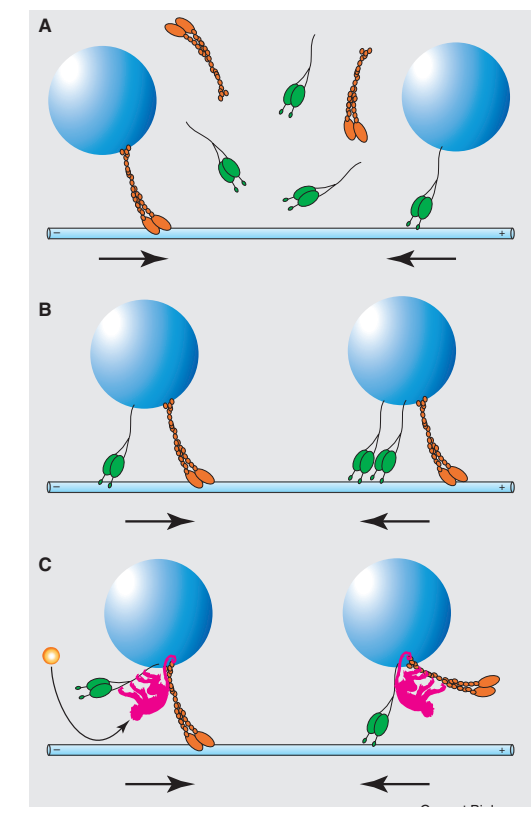

Figure 13: Possible mechanisms for bidirectional transport. Plus-end directed motors (orange) and minus-end directed motors (green) move a cargo (dark blue) along a MT (light blue). (A) A single motor species can be attached. (B) Tug-of-war of different motor species. (C) A coordination machinery determines moving direction. From [388].

Even with net drift motion, bidirectional motion can be more efficient than unidirectional transport in a crowded environment as the axon: When the cargo is blocked by an obstacle, stepping back allows to try to find another path around.

\subsubsection{Tug-of-war}

It has been shown that in general, the two types of opposite motors are attached at the same time on the cargo [388, 156]. Then two scenarios can be considered [151, 327, 40] (Fig. 13(B) and (C)). Either there is a - yet not fully specified mechanism that coordinates the motion of motors, so that motors of one type are inhibited while the others pull the cargo and the other way round, or bidirectional motion emerges spontaneously through stochastic fluctuations of the number of motors attached on the cargo at a given time. The latter mechanism is referred to as "tug-of-war"

In the tug-of-war picture, the attachment of motors of each species results from a random procedure, and it is only because of random fluctuations that one or the other species will take over and carry the cargo towards its preferred direction. As a result, the cargo will go back and forth, possibly performing large (compared to the typical motor step size) excursions in each direction [156]. An experimental observation in favour of this scenario was made on endosomes which were found to be elongated when pausing [351]. This could be a consequence of the pulling of opposite teams during tug-of-war episodes.

Some theoretical models have been proposed, in which the number of cargo-bound motors of each type that are also attached to the MT are taken as internal degrees of freedom [199, 262, 263]. A mean-field version, in which forces are equally shared among attached motors of the same type, showed that drift motion could be spontaneously obtained when one type of motors pulls the cargo strongly enough to have the motors of the other type detach [266, 399]. The resulting high motility states obtained by forcing the minority motor species to detach from the filament, and characterized by a symmetric bimodal (or trimodal) distribution of velocities, can be seen as some kind of spontaneous synchronization and do not require the action of an external factor. However in this model, though the attachment and detachment of motors is

\footnotetext{
${ }^{9}$ Tug-of-war refers to a game where two teams pull in opposite directions on one rope, and try to force the other team into their own camp. Tug-of-war contests were organized at the Olympic Games from 1900 to 1920
} 
considered, the explicit dynamics of motors along the filament is not described, which is required in order to go beyond the mean-field approach and to compute the forces individually for each motor.

Indeed, stochastic models with explicit position tracking of the motors have been proposed, in which the individual forces felt by the individual motors which are located at specified positions on the MT are taken into account [214, 215, 194]. Such models were developped for cargos driven either by one type of motors [209, 47] or by two types of opposite motors $[215,48,49,194]$ - the latter being the case of interest here for us. The comparison between these stochastic models and the mean-field model revealed strong differences in the dynamics [48]. Indeed, when motor position is explicitely considered, it turns out that motors which are oppositely directed to the dominant motor species are not automatically disrupted from the MT. Thus the efficiency of the tug-of-war mechanism is drastically reduced, in the sense that the symmetric bimodal distributions observed in the mean field model do not exist anymore [195]. This is not necessarily a drawback, as these symmetric bimodal distributions have never been clearly evidenced experimentally. Still, the stochastic model reproduces well that the tug-of-war leads to anomalous diffusion in agreement with some experimental observations[213, 68, 308, 318]. Indeed, at very short time scales and if thermal fluctuations are taken into account, subdiffusion is observed as a result of the trapping of the cargo by oppositely pulling motors [193]. At larger time scales (typically tens of $\mathrm{ms}$ ), there is a crossover towards superdiffusion which indicates that cargo-mediated coupling induces some cooperativity between the motors [192]. At even larger time scale, one expects to recover pure diffusion, but usually network effects come into play first.

Besides, the fact that the response of motors to external factors (load, [ATP], etc) depends on their type [243] provides a way for the cell to control the bias of the cargo random walk. Indeed, a change for example in the viscosity of the surrounding medium can modify the bias in a non trivial way, and even reverse it, by changing the balance between motors' teams [192]. This mechanism could in particular allow cargos to escape from crowded areas if the role of obstacles could be assimilated to a large effective viscosity.

Some complementary mechanisms, for example through hydrodynamic interactions, could also play a role in obtaining correlated motion [238, 239].

\subsubsection{Coordination-based scenarios}

Another hypothesis would be that, instead of resulting from a purely random procedure as in the tug-of-war, motion would be produced through some coordination mechanism. Indeed the available experimental data are consistent with a number of regulatory mechanisms, which we will partially review now.

The perhaps most direct option to control cargo transport is by the cargo itself: Through specific proteins that are located at the cargoes' surface it controls the docking or the activity of the attached motors [186]. It has been suggested that this mechanism could orient synaptic cargos preferentially towards MT or actin networks [327].

Another mechanism, which is also based on the selective activation of the different motor species, is provided by molecules which are potentially able to coordinate the motor activity [112]. Dynactin, for example, usually turns the minus-directed motors off if the plus-end directed motors are active [150]. Thus binding of dynactin should lead to a persistent plus-end directed motion of the cargo [243]. A crucial regulatory factor in this process is the hungtingtin protein, that interacts with dynein, dynactin and kinesin. It was shown in primary cultures of neurons that the phosphorylation of huntingtin promotes anterograde transport, while retrograde transport is favored when hungtingtin is not phosphorylated [81].

Finally it is also possible to alter the properties of the MT filaments via decoration with so-call MT associated proteins (MAPs). MAPs as for example the tau protein can influence the binding affinity of molecular motors, which would have an influence on the run length of the cargo, since the average number of motors that is attached to the MT is reduced.

Whatever the mechanism is, cargos do undergo directed motion in cells. Cooperative effects of the motors are observable, leading for example to increased processivity of a transported cargo and higher stalling forces than for individual motors which has been shown experimentally [244, 34]. Cooperation (spontaneous or mediated) between motors thus enhances long-range transport of individual cargos.

\subsubsection{Transport of large cargos}

The transport velocity of cargos may depend on their size, the larger ones travelling at smaller velocities [348].

One important example of a large cargo is mitochondria, typically measuring $10 \mu \mathrm{m} \times 0.2 \mu \mathrm{m}$. Mitochondria are present in almost every eucaryotic cells and can be transported along microtubules. Remarkably mitochondria are also to 
be transported along the axon [288, 162, 280] although their size is much larger than the normal interdistance of MTs in the axon $(\sim 20-30 \mathrm{~nm}[72])$.

Several mechanisms have been proposed which enables mitochondria to move in this crowded environment. There may for example be mechanisms based on the dephosphorylation of MAPs that would allow MTs interdistances to grow so as to leave a free tunnel through which the vesicle could pass [340]. It is also possible that MTs locally depolymerize and open this way free space for giant vesicles and mitochondria.

To conclude, though much progress has been done to understand the mechanisms underlying motor-cargo complexes, their transport is far from being fully understood. Since most of the experiments are carried out in vivo, the actual configuration of the motors attached to a given cargo [40] as well as the influence of e.g. regulatory proteins is $a$ priori not known. Therefore it would be of great interest to carry out in vitro experiments, where it is possible to control the configuration of molecular motors that are attached to the cargo and the environmental parameters. Actually, a recent discovery on how to handle mammalian dynein in motility assays [250] should allow to build in the near future in vitro experiments with two types of motors and to study bidirectional cargo motion in much more controled settings. Much will probably be learned on these systems in the coming years.

\subsection{Dynamics of molecular motors on filament networks}

In this review, we have mostly considered transport at the scale of a single filament. However, in order to describe intracellular transport at larger scales, it is necessary to take into account the global structure and dynamics of the network. An important set of work has been done on this subject using non-linear continuous models. Several reviews have been already published on this subject, to which we refer the reader for further information [177].

In the following, we shall rather address this subject using microscopic models.

In $[145,146]$ the dynamics of self-driven particles on regular and inhomogeneous networks embedded in a two dimensional diffusive environment has been investigated. The dynamics of the particles alternates between diffusive motion of unbound particles and driven motion if the particles are attached to filament like structures. The active transport of particles leads generically to the formation of particle clusters at the intersection of filaments. Interestingly the size-distribution of the particle clusters depends qualitatively on the chosen geometry of the filament network: On regular networks one typically observes exponentially decaying cluster distributions at low densities, i.e. one can attribute a typical scale to the clusters. By contrast, on inhomogeneous filament networks typically algebraic cluster-size distributions are observed in a large parameter regime.

The origin of the scale-free cluster-size distribution can by deduced from the following consideration: Clusters nucleate if two particles encountering at an intersection of two filaments block each other such that they cannot move, while other particles running towards the intersection form queues. If the particle density exceeds a critical density $\rho^{*}$, queues form that cover multiple filaments and induce branching of queues into large clusters. Now each branch contributes to the particle support of the cluster. Since the number of filaments a cluster covers is proportional to its size, the growth rate of a cluster is also proportional to its size. Dynamics of this kind can be described by a Yule process [397] which yields a power law distribution $P(m) \sim m^{-\gamma}$ with an exponent depending on the microscopic parameters. For disordered filaments at moderate particle densities, it turned out that the exponent describing the decay of the cluster-size distribution depends on the particle density and the typical mesh-size of network, while dependence on the other model parameters is rather weak.

We have seen that the distribution of particles on the filament network was quite different depending whether particles were transported through active transport on the one hand, or random walk and aggregation processes on the other hand. There is a need to understand these differences more thoroughly.

In view of the application to intracellular transport it would be interesting to study the aggregation on dynamic networks as well, since the steady reorganization of the filament network may have a strong influence on the formation of particle clusters (We shall see in section 7.3 such an example). It is also possible that the motors themselves modify the dynamics of the network. For example kinesin 8 was shown to promote MT depolymerisation, a feature that could play a role in length regulation of MTs.

Indeed, this would contribute to the understanding of complex questions such as the potential toxicity of nanoparticles. Nanoparticles are known to pass the cell membranes [279]. It is important to have some knowledge about the way they are transported and where they are aggregated inside the cell, to determine whether they can have an impact on cell functions.

To conclude this chapter, though a complete description of cargo motion requires the full description discussed in this chapter, at a coarse grained scale, cargo-motors complexes could themselves be represented as single particles (and 
backstepping should then be included). Thus in the next chapters, though most of the time single particles will be thought as single motors, it is also possible to consider that they represent a whole cargo-motors complex in a more coarse grained description.

\section{Exclusion Processes}

When trying to model such complex issues as intracellular transport, one is usually confronted with several difficulties. First of all, there is the huge complexity of the problem. As we shall discuss in section 7.1 for the example of motordriven axonal transport, transport processes are typically the result of the dynamics of many different proteins interacting in ways which are not fully understood. This lack of knowledge makes modeling a challenging task. But even if we had a perfect knowledge about the intracellular processes on a molecular level, it would still be hopeless to model motor-driven transport in every detail. Therefore, it is necessary to describe motor-driven transport on a coarse grained level or even by a continuous approach.

In this chapter we will discuss a stochastic approach to motor-driven intracellular transport. Stochastic modeling has proven itself as a powerful tool in systems with complex interactions on a microscopic scale. A convenient example is the Brownian motion of a small particle in a suspension. Although the collisions between the molecules of the liquid and the particle are not explicitly considered, the stochastic treatment of the random particle dynamics describes well the real system. By describing the dynamics of the system in terms of stochastic transitions between different states, the microscopic interactions are characterized only by giving the transition rates. This approach hence corresponds to a treatment on a coarse-grained scale and overcomes the problem of lacking knowledge or extreme complexity.

In the past, stochastic lattice gas models have been used in a wide range of active transport phenomena, e.g., pedestrian dynamics [323, 373, 79], car traffic [76, 271, 13] and intracellular processes [235, 77, 228]. Lattice gas models are discrete in space, i.e. particles which occupy the sites of the lattice hop stochastically from one site to another. The particles move according to hopping rules and rates which are chosen to mimic the properties of the modeled system. In active transport models, the hopping rates may not be related to a potential via the detailed balance condition. Therefore generic nonequilibrium phenomena, as for example macroscopic currents in the stationary state, are frequently observed in transport models. All the models which we will discuss in this review are non-equilibrium models.

In the case of intracellular transport, stochastic lattice gases are an appropriate choice because the hopping of particles on the lattice can be directly understood as a step of a molecular motor like kinesin or dynein along the MT. The different stages of the mechanochemical cycle during which ATP is converted to ADP and mechanical work is produced (see section 3.1.1) can be summarized in a single hopping rate, hence drastically simplifying the problem (though in a more detailed description it is also possible to retain some features of the mechanochemical cycle as has been discussed in section 3.2.2).

We shall present in this section the so-called totally asymmetric simple exclusion process (TASEP) ${ }^{10}$ as a minimal model that includes important features of the dynamics of molecular motors : the stepwise, directed, and stochastic motion of single motors as well as the steric interactions between motors are described. Nevertheless the original TASEP is an incomplete model for transport even on the coarse grained level, since. e.g., the attachment and detachment of motors is not included. Therefore the TASEP should be seen in the context of this review as a reference on which more detailed approaches can be built.

Before presenting more sophisticated models which have been introduced in order to describe intracellular transport driven by molecular motors, we shall summarize in this section some basic properties of exclusion processes, and some variants whose properties will be useful in order to analyse intracellular transport models. At the same time, driven stochastic lattice gases have proven to exhibit a rich phenomenology already in one-dimensional systems making them an interesting object of study from the point-of-view of statistical physics. In fact, phase transitions in one-dimensional systems without long-range interactions can only be observed in non-equilibrium systems.

\subsection{Totally asymmetric simple exclusion process (TASEP) \\ 4.1.1. Model definition}

The TASEP is probably the most extensively studied model of stochastic transport. It is defined on a one-dimensional lattice with $L$ discrete sites that can either be vacant or occupied by a particle (figure 14). The state of the system at a given

\footnotetext{
${ }^{10}$ The TASEP model was first proposed in the context of mRNA translation and synthesis of proteins [235]. We will not detail this application, as reviews on the subject already exist $[401,73]$.
} 


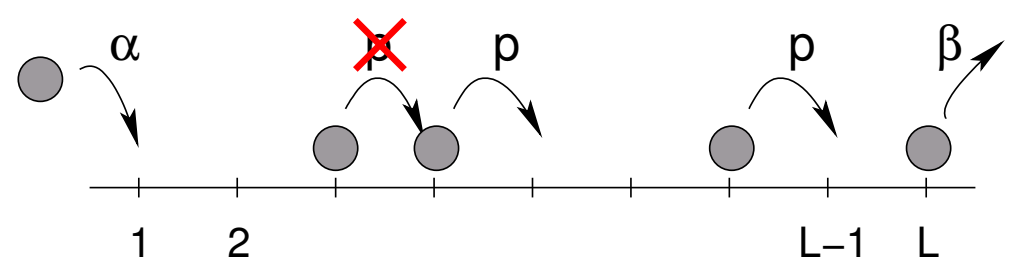

Figure 14: The TASEP with open boundary conditions. A particle hops to the next site on the right if that site is empty at rate $p$. If vacant, the first site of the system is filled from the reservoir at rate $\alpha$ and a particle on the last site can leave the system at rate $\beta$.

time is given by $\left\{\tau_{i}\right\}_{i=1}$ to $L$, where $\tau_{i}$ gives the occupation of site $i$ taking values 0 or 1 if that site is vacant or occupied, respectively. A particle hops stochastically to a site on its right with rate $p$ if that site is vacant, i.e., no site can be occupied by more than one particle at a time. Hopping to the left is prohibited in this model but possible in other simple exclusion processes as the symmetric simple exclusion process (SSEP) or the asymmetric simple exclusion process (ASEP). For the purposes of transport by molecular motors, the totally asymmetric variant is often appropriate because backward steps of motors as kinesin-1 are rare and can be neglected (see section 3.1.1).

Two different boundary conditions are usually considered: Under periodic boundary conditions, the system is closed into a ring so that a particle which hops from site $L$ arrives at site 1 . This choice obviously exhibits conservation of particles and it can be shown that in this geometry, every allowed configuration has the same probability of occurrence [251, 89]. Open boundary conditions are realized by coupling the chain to two boundary reservoirs. Usually one considers boundary reservoirs of infinite capacity. Particles enter the system with rate $\alpha$ at the left boundary if the first site is empty and leave the system with rate $\beta$ from the last site if it is occupied.

\subsubsection{Update scheme}

In order to simulate the time evolution of the TASEP, one has to chose an update scheme, that is one has to define when and in which order the particles are attempting to hop forward.

Let us first make a general remark about systems at equilibrium (which is not the case of the TASEP). In equilibrium statistical mechanics, lattice models are usually defined by the different states of the lattice, the interactions between the state variables and the geometry of the lattice. From this definition, a partition function can be calculated, which fully determines the equilibrium state of the system. In order to explore numerically the equilibrium properties of these models one can apply different Monte Carlo schemes which sample stochastically the configuration space. It can be shown that all ergodic Monte Carlo algorithms that fulfill the detailed balance condition lead to the same stationary state, i.e. the equilibrium state of the system.

For non-equilibrium systems as those considered in this review, the model is defined by a master equation. This implies that the update scheme is part of the definition of the model. In the case of the TASEP, as for many other nonequilibrium systems, it can be shown that a unique stationary state exists for a given update, but the stationary state and its properties can differ from one update to another one [300,191]. We shall present now the most widely used types of updates that have been considered to model stochastic transport.

Random sequential update. If we were considering a continuous time evolution, the distribution of time intervals between two events would follow an exponential law. The most appropriate numerical method for stochastic processes continuous in time would be the Gillespie algorithm [138, 139], in which these exponential time interval distributions are realized. However, in the special case where all rates are of the same order of magnitude and where one considers a large system, it is more convenient to use a discrete time algorithm, namely the random sequential update.

For the TASEP, the random sequential update can be implemented as follows. At each micro-time step, a link (separating two neighboring cells) is chosen. If there is a particle on the left and no particle on the right of this link, the particle is moved forward with probability $p^{11}$. If the chosen link corresponds to the entrance (exit), then a particle is injected on the first site (removed from the last site) with probability $\alpha(\beta)$.

\footnotetext{
${ }^{11}$ Computationally it is more efficient to to choose a particle (or a hole for densities $\rho>1 / 2$ ) at each micro-step, and to move it forward (backward) with probability $p$ if the target site is empty. For a closed system, with fixed number $N$ of particles, the microstep is constant and lasts $1 / N$. For an open system, the microstep duration depends on the (varying) number $N$ of particles.
} 
The duration of a microstep is $1 / \#$ links ${ }^{12}$, so that on average each link is updated once per unit time step. The set of the \#links updates that occur during one unit time step is usually called a sweep.

Though it is not strictly identical to a continuous time dynamics, the random sequential update gives a very similar behavior for large system sizes, in particular when simple quantities such as current and densities are measured. Still, one must be aware that discrepancies may arise when more sensitive quantities are measured, such as generating function or higher order cumulants (see section 6 of [90] for an example). An important feature of this update is that one particle may be chosen several times in a row while another one may be ignored during several time units, which leads to non-negligible current fluctuations even at low densities. It is this update that has been considered in most fundamental studies, for which TASEP was taken as an archetype of non-equilibrium systems.

It should be noted that for the random sequential update, time can be rescaled. Indeed, the only effect of changing the physical duration of the unit time step is to change the numerical values of the rates in numerical time units - so that they remain unchanged in physical time. Usually, one chooses the time step so that the largest rate defined in the system is equal to one (in numerical units). This allows to have the largest ratio of acceptance for the transitions (the time scale must be chosen so that rates are not larger than one, as they must be interpretable as probabilities).

Parallel update. For some applications, a certain regularity of the particle motion exists, which limits for example the number of steps that can be carried out in a given time interval. One possibility to obtain this regularity is to use a parallel update scheme, an update which is widely used in order to model e.g. road traffic [271, 111]. For these applications not only the regularisation of the speeds is of importance but also the implicit introduction of a reaction time, which is necessary in order to represent traffic states in a realistic way.

In the parallel update $[116,324,392]$, all sites are updated at the same time. The state of a given site at time $t+1$ depends on the state of this site and its neighbors at time $t$, through -when applied to the TASEP- the rules introduced in section 4.1.1. In contrast to the random sequential model $p$ now is a hopping probability rather than a rate. This implies that for $p=1$ the bulk dynamics of the particles is deterministic $[362,85,120]$ in contrast to the random sequential model where the particle motion is stochastic for any finite hopping rate.

The parallel update introduces a time step, which now cannot be arbitrarily rescaled. The time step can be interpreted as a reaction time or as a time which is needed in order to carry out the microscopic step. Another feature of this update is that not all the states of the system are accessible through the dynamics. The non-accessible states were called "Garden of Eden" states [324] (Note that the definition of non-accessible states requires to include not only the positions but also the velocities of particles in the definition of the states). They are related to the existence of strong short-ranged correlations. In case of the TASEP the GoE-states introduce a particle-hole attraction, which is more pronounced as $p$ is closer to 1 and may lead to high current states of alternating empty and occupied states.

Conflicts naturally arise with parallel update if two-dimensional lattices are considered, as this is the case for pedestrian traffic: Two particles may attempt to hop to the same site at the same time, which is forbidden by the exclusion principle. Therefore one has to introduce additional stochastic or deterministic rules, which assign the priority to one of the particles that try to occupy a given site. One may argue that in some case, these conflicts should not be considered as an artifact of the model but are also present in the real systems, e.g. when several pedestrians are trying to walk through the same door [189, 325]. Anyhow, the presence of conflicts requires to supplement the update rules with ad-hoc conflict-solving rules which can be deterministic or stochastic. These rules may not be that relevant at intermediate densities, and their choice can be quite arbitrary. This was enough to trigger the development of new updates that would at the same time be sequential and have bounded fluctuations.

Shuffle updates. In the random shuffle update ${ }^{13}$ [390, 391, 349, 201], at each time step, each particle is updated exactly once, but the order in which particles are updated is chosen randomly at each time step.

If the order of update is chosen once for all at the beginning of the simulation, this update will rather be called frozen shuffle update $[15,14,16]$.

Random or frozen, the shuffle update is a sequential update, and as a consequence no conflict arise. As in the parallel update case, the time step cannot be trivially rescaled. Fluctuations of the particle velocities are reduced compared to random sequential update by the fact that each particle is updated exactly once per time-step.

\footnotetext{
${ }^{12}$ The notation \#links stands for the number of links.

${ }^{13}$ Note that in some communities (for example working on agent based models), the random shuffle update is called random sequential update. This is a source of confusion that the reader must be aware of. In this review, we keep the definitions usually used in physics.
} 
In the case of the frozen shuffle update, the fact that that for a given particle, there is exactly one time unit between two updates reduces even further the fluctuations and yields smooth motion in the free flow phase.

Some other updates that we will not discuss further here have been proposed, such as sequential update ordered backward or forward in space $[45,55,54,291,116]$ or sublattice update $[158,121,287,290]$. They can be seen as particular realisations of the frozen shuffle update.

Updates for intracellular traffic. For the frozen shuffle update and parallel update, two hopping attempts are always separated by exactly one unit time. This can be relevant for phenomena that proceed from an internal clock, as stepping for pedestrians, and may have applications in biology for agents having a cyclic behavior.

In the case of molecular motors, the delay between two steps is mainly due to the delay for the arrival of a new ATP. Within the stepping cycle, the ATP hydrolysis is fast enough so that it does not yield a significant incompressible delay value. The limiting rate corresponds rather to the dissociation of the motor head from the MT after ATP hydrolysis [137], but its value of $20 s^{-1}$ is usually considered to be fast enough to be neglected. As a consequence, the stepping behavior of molecular motors is rather described by a random sequential update.

Note that some papers [215] have been using parallel update, but with so small time steps that it becomes equivalent to a random sequential update. Indeed, the introduction of an intrinsic time scale will have an impact only if it is not negligible compared to all the inverse rates.

In this review, if not otherwise stated, it will always be a random sequential update that will be considered.

\subsection{Theoretical results for the TASEP with random sequential update}

As the most widely used update scheme in this review will be the random sequential update, it is for this update that we shall now give a non exhaustive survey of the results obtained for the TASEP. Other complementary reviews can be found for example in $[89,241]$. Here we shall concentrate on the properties that will be of relevance for intracellular traffic.

\subsubsection{Time evolution of the density}

The evolution equation for the particle densities $\left\langle\tau_{i}\right\rangle$ in the bulk are given by

$$
\frac{d\left\langle\tau_{i}\right\rangle}{d t}=p\left\langle\tau_{i-1}\left(1-\tau_{i}\right)\right\rangle-p\left\langle\tau_{i}\left(1-\tau_{i+1}\right)\right\rangle=J_{i-1, i}-J_{i, i+1},
$$

where $\langle\ldots\rangle$ denotes the statistical average, $d t$ is the microscopic timestep, and $J_{i, i+1}$ is the average current accross bond $(i, i+1)$. For an open system, these equations become at the boundaries:

$$
\begin{gathered}
\frac{d\left\langle\tau_{1}\right\rangle}{d t}=-p\left\langle\tau_{1}\left(1-\tau_{2}\right)\right\rangle+\alpha\left\langle 1-\tau_{1}\right\rangle, \\
\frac{d\left\langle\tau_{N}\right\rangle}{d t}=\left\langle\tau_{N-1}\left(1-\tau_{N}\right)\right\rangle-\beta\left\langle\tau_{N}\right\rangle .
\end{gathered}
$$

\subsubsection{Current-density relation}

Investigating on the efficiency of motor-driven transport, the current across a bond between two neighboring sites is a quantity of high interest and is defined as the average number of particles crossing that bond in one unit of time. In the stationary state, since the TASEP obeys local conservation of particles, the average current is also conserved throughout the system and thus is equal for all bonds to

$$
J:=J_{i, i+1}=p\left\langle\tau_{i}\left(1-\tau_{i+1}\right)\right\rangle,
$$

where $i$ denotes the starting site of the hopping move through bond $(i, i+1)$. In a product-state or mean field approximation, the averages over products of state variables are assumed to factorize $\left\langle\tau_{i} \tau_{j}\right\rangle \approx\left\langle\tau_{i}\right\rangle\left\langle\tau_{j}\right\rangle$. Let $\rho_{i}=\left\langle\tau_{i}\right\rangle$ be the average density at site $i$. As the periodically closed system exhibits translational invariance, we have $\rho_{i}=\rho \forall i$. Applying the mean field approximation and translational invariance to (12), the unique current-density relation

$$
J(\rho)=p \rho(1-\rho)
$$

is obtained which is actually exact for periodic boundary conditions in the continuum limit [92] and identical to the one obtained for large systems with open boundaries as discussed hereafter. Its maximum is at $\rho=1 / 2$ where the corresponding current is $J=p / 4$, a value which may serve as benchmark to evaluate the transport capacity of other models. 


\subsubsection{Phase diagram}

In the case of open boundary conditions, which will be considered in the rest of this section, two infinitely large particle reservoirs are imagined at both ends of the lattice with density $\rho_{0}=\alpha / p$ for the reservoir on the left and density $\rho_{L+1}=$ $(1-\beta) / p$ for the reservoir on the right. The first site of the lattice can hence be filled at rate $\alpha$ if it is empty and a particle on the last site leaves the lattice at rate $\beta$ (figure 14). In the stationary state, the system with open boundary conditions has been solved by Derrida et al. using a matrix product ansatz [92] and simultaneously by Schütz and Domany [334] by solving the exact recursion relations found in [91], confirming some partial results obtained from a phenomenological approach by Krug [210].

An important property of the TASEP is its particle-hole-symmetry, i.e., the identical behavior of the model under certain symmetry operations:

$$
\tau_{i} \leftrightarrow 1-\tau_{i}, \quad \alpha \leftrightarrow \beta, \quad i \leftrightarrow L+1-i .
$$

These symmetry operations also imply a symmetry of the density profile:

$$
\rho_{L+1-i}(\alpha, \beta)=1-\rho_{i}(\beta, \alpha) .
$$

Thanks to this property, the whole phase diagram can be deduced from considering only half of the parameter space $(\alpha, \beta)$.

In contrast to the system with periodic boundary conditions, the TASEP with open boundary conditions shows interesting non-trivial behavior. Depending on the entrance and exit rates $\alpha$ and $\beta$, three different phases can be reached [43] in the stationary state:

- Low-density phase (LD): For $\alpha<\beta$ and $\alpha<p / 2$, the entry rate dominates the system's behavior and determines current and bulk density:

$$
J=\alpha(1-\alpha), \quad \rho=\alpha .
$$

At the right boundary, the density profile exhibits a boundary layer which decays exponentially to $\alpha$. If $\beta>p / 2$ the exponential decay is additionally modulated by a power law.

- High-density phase (HD): For $\alpha>\beta$ and $\beta<1 / 2$, the exit rate limits the current in the system and creates a queue of particles in front of the exit which extends through the whole system except for the boundary layer on the left end of the system.

$$
J=\beta(1-\beta), \quad \rho=1-\beta, \quad \text { for } \alpha>\beta \text { and } \beta<1 / 2 .
$$

Again, the left end boundary layer decays to the bulk value, with the characteristic of the decay depending on the value of $\alpha$ analogous to the low-density phase.

- Maximal-current phase (MC): For $\alpha>p / 2$ and $\beta>p / 2$, a maximal current is reached which is independent of the exact values of $\alpha$ and $\beta$.

$$
J=p / 4, \quad \rho=1 / 2 .
$$

The boundary layers in this phase decay algebraically toward a density of $\rho=1 / 2$, leading to long-range boundary layers from both sides.

The expressions given in Eqs (16), (17), and (18), are valid in the limit of large systems. For smaller systems, finite size corrections have to be added [334]. The phase diagram of the TASEP in the stationary state and for large systems is shown in figure $15 \mathrm{~A}$ and typical density profiles of the three phases can be seen in figure $15 \mathrm{~B}$.

The transitions from the low/high-density to the maximal-current phase are continuous: The bulk density approaches $1 / 2$ when arriving at the transition line from the low/high-density phase. In contrast, the transition line separating lowdensity from high-density phase is a first-order transition: The density changes abruptly from $\alpha$ to $1-\beta$ when crossing the transition line thus giving a total density change of $1-2 \alpha$. The current nevertheless is continuous upon crossing of the transition line. On the transition line at $\alpha=\beta<p / 2$, both reservoirs try to impose their density on the system leading 
A

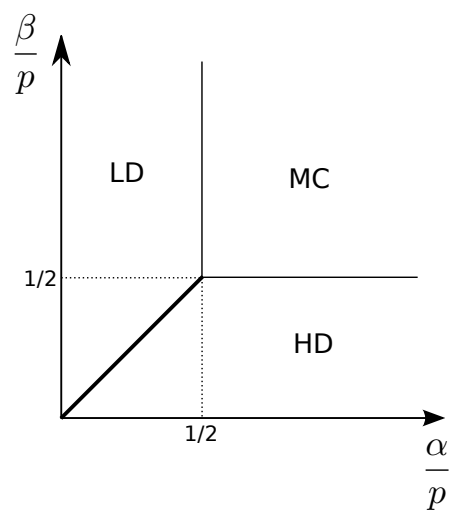

B

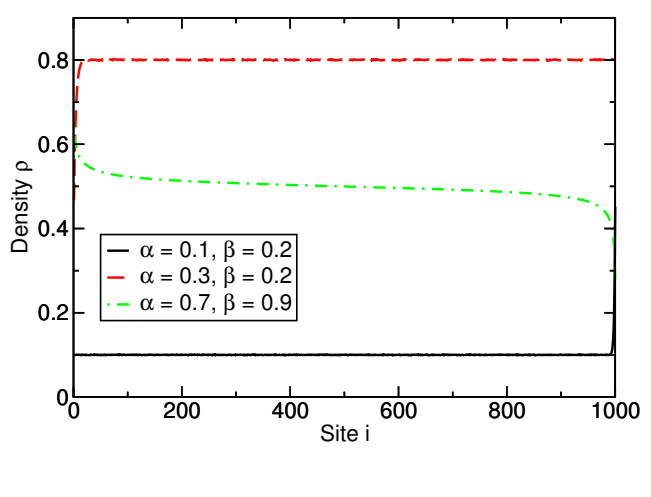

Figure 15: Phase diagram and density profiles of the TASEP with open boundary conditions. (A) Phase diagram of the TASEP with its three phases: low-density (LD), high-density (HD) and maximal-current (MC). The thick line between LD and HD indicates a first order transition, thin lines at the HD/MC and LD/MC interfaces are second order. The thin dotted lines indicate changes of the boundary layer while the bulk behavior remains unchanged. (B) Representative density profiles for a TASEP of system size $L=1000$ for the three phases with $p=1$. LD at $\alpha=0.1, \beta=0.2$ (solid black line), $\mathrm{HD}$ at $\alpha=0.3, \beta=0.2$ (red dashed line) and MC at $\alpha=0.7, \beta=0.9$ (green dash-dotted line).

to flat profiles emanating from both ends of the system which are connected by a shock front. This shock diffuses through the system, i.e., it performs a random walk along the lattice, leading to a linear averaged density profile.

In the LD and the HD phase, the shock connecting the densities of the two reservoirs has a non-zero average velocity causing it to be driven to the right and left boundary, respectively, where it forms the boundary layers mentioned above. We shall see in the next section how a phenomenological description of the motion of the shock can be developped.

In summary, due to the conservation of particles and current, the boundaries are able to determine the bulk properties in the TASEP. It is remarkable to observe this rich phase diagram despite the simple structure of the model. This is a direct consequence of the driving which keeps the system out of equilibrium.

It should be kept in mind that also partial asymmetric dynamics can be considered (ASEP), to take into account the fact that motors may have a non-vanishing probability to back-step (see section 3.1.2). In most cases only minor differences are observed compared to the TASEP. It is only in the special case where the particle reservoirs drive the system against the bias [44] that qualitatively new behavior is observed: The net current exponentially decreases with the system size.

In the remaining of this section, we shall consider only the TASEP version.

\subsection{Domain wall approach}

We have mentionned above that in part of the phase diagram (actually for $\alpha$ and $\beta<p / 2$ ), each boundary reservoir tries to impose its density to the system, resulting into two flat domains separated by a shock. This observation can be used to provide a coarse grained description of the system dynamics (valid in the aforementionned part of the phase diagram): the so-called domain wall approach [205, 287].

This phenomenological approach is complementary to the numerous exact results that have been obtained for the TASEP: Apart from giving a more physical intuition of the mecanisms involved, we shall see that the domain wall description is valid not only for stationary but also non stationary states, and can be more easily extended to variants of the TASEP.

But first we shall describe it in the case of the TASEP. Each boundary condition of the system implements a boundary reservoir of a given density. The actual density depends on the precise implementation of the model. In case of the TASEP with random sequential update the left reservoir inserts particles with density $\alpha$ and the right reservoir holes with density $\beta$ leading to a particle density of $1-\beta$. If $\alpha \neq 1-\beta$ the particle density of the two domains is different such that in the coarse grained picture the density is discontinuous at the point where the two domains meet, i.e. the domains are separated by a shock.

In the domain wall description, the smooth shocks that separate the free flow and jammed regions are considered as sharp discontinuities. Simple considerations based on mass conservation yield the velocity of the shock in terms of the 
fluxes and densities on both sides, as we shall explain now. Let $\rho_{i}$ be the particle density at site $i$ and $j_{i+1 / 2}$ the current from $i$ to $i+1$. Then the conservation of mass implies

$$
\frac{d \rho_{i}(t)}{d t}=j_{i-1 / 2}(t)-j_{i+1 / 2}(t)
$$

The velocity of the shock far from the boundaries in the continuum limit can be obtained from the continuity equation (19)

$$
v_{s}=\frac{j_{+}-j_{-}}{\rho_{+}-\rho_{-}}
$$

where $\rho_{ \pm}$and $j_{ \pm}$denote the density and current of the domain left $(-)$or right $(+)$from the shock. Due to the stochastic input of particles, the motion of the shock is non-deterministic.

For the random sequential update, it turns out that it is appropriate to describe the dynamics of the shock as a biased random walk with hop rate $D_{+}$to the right and $D_{-}$to the left. These hopping rates can be related to the drift velocity of Eq. (20) by $D_{+}-D_{-}=v_{s}$. This relation cannot fully determine the hopping rates. Those can be partially inferred from limit cases and were postulated to be given by [205]

$$
D_{+}=\frac{j_{+}}{\rho_{+}-\rho_{-}} \quad \text { and } \quad D_{-}=\frac{j_{-}}{\rho_{+}-\rho_{-}} .
$$

By mapping the dynamics of the domain wall dynamics to a biased random walk on a finite lattice with reflecting boundary conditions, the dynamics and the stationary distribution of the domain wall positions can be evaluated. The latter is given by

$$
P(i)=\mathcal{N}^{-1} \exp \left(-\frac{L-i}{\xi}\right)
$$

in the low density phase $(\alpha<\beta)$ and

$$
P(i)=\mathcal{N}^{-1} \exp \left(-\frac{i}{\xi}\right)
$$

in the high density phase $(\alpha>\beta)$. In these expressions, $\mathcal{N}$ is a normalization factor $\mathcal{N}$ and $\xi$ is a localization length given by

$$
\xi^{-1}=\left|\ln \frac{D_{+}}{D_{-}}\right|=\left|\ln \frac{\beta(1-\beta)}{\alpha(1-\alpha)}\right| .
$$

The stationary density profile can be derived from (22-24). It is found to be in agreement with the exact expression valid in the large system size limit [334], with in particular the same localization length $\xi$. This agreement can be seen as a validation a posteriori of the choice (21) for $D_{+}$and $D_{-}$.

The first order transition between high- and low-density phase that occurs at $\alpha=\beta$ is accompanied by a divergence of the localization length. This illustrates the non-equilibrium nature of the boundary induced phase transition. It should be noticed that the domain wall theory is only valid if the capacity of the boundary reservoir does not exceed the capacity of the chain. For values of $\alpha / p>\frac{1}{2}$ or $\beta / p>\frac{1}{2}$ the structure of the domains is non-trivial and the simple random walk picture cannot be applied.

Despite this limitation the domain wall approach is very usefull. First of all it gives a simple and intuitive physically based description of the first order transition along the $\alpha=\beta$ line. Moreover, while exact results were obtained mostly for stationary states, the domain wall approach allowed to predict with a very good accuracy several non-stationary features [103, 272, 321]. Eventually, while the DW approach is meant to be valid only in the large system size limit, actually it works remarkably well for finite systems if not too close to the maximal-current phase (see [321] for a comparison with systems of a few tens).

The domain wall approach can also be applied to a large set of variants of the TASEP which have a continuous fundamental diagram, while exact techniques usually are much more difficult to generalize. We shall see later how the domain wall approach allowed in particular to understand multilane TASEP models [326] (section 5.2.2) and models without particle conservation in the bulk (section 6.2).

It should be underlined that, though mass conservation gives a firm base to Eq. (20), the expression for the hopping rates $(21)$ is a postulate. In the case of the random sequential update, this choice was confirmed by the agreement between 
the predictions of the DW theory [205] and the exact solution [334] or numerical results [272, 321]. It was noticed in [205] that the DW approach developped for the random sequential update was also predicting correctly the localization length in the case of parallel update in the limit of small $\alpha$ and $\beta$ [362]. However, in general, the DW approach as presented above gives only approximate results when applied to other updates than random sequential (see [14] for an example in the case of the frozen shuffle update [15]). Actually the DW theory postulates that the motion of the wall can be described by the master equation of a biased random walk [103] with the hopping rates (21). While this is relevant for the random sequential update, it can be shown [80] that in the case of a deterministic TASEP with parallel update, a non-Markovian evolution equation has to be used: There is a memory effect over one time-step. Note that in this special case, the domain wall theory is not phenomenological anymore, but can be made exact even at the microscopic scale.

\section{Variants of TASEP}

It is out of scope to review all the litterature on variants of TASEP. Here we selected a few of them that contain some ingredients relevant for biological transport.

\subsection{TASEP with on-off gates}

\subsubsection{Single lane TASEP with dynamical gate}

In the previous section, the disorder was given as a characteristic of the model, and thus independant of the evolution of the system. However, it may happen that the properties of the transport network depend on the occupation state of the network by particles.

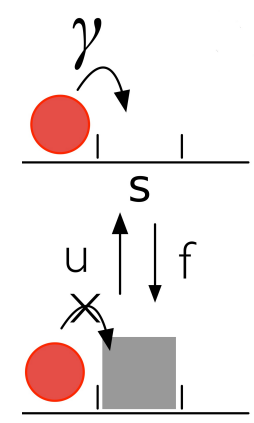

S

Figure 16: Definition of the dynamics of a single site dynamical defect located in site $s$. Only two sites of the lattice are represented. Particles cannot enter site $s$ when it is closed, while the closing of site $s$ is not possible when it is occupied by a particle. Within this constraint, opening and closing of site $s$ occur respectively with rates f and u (these symbols were chosen in reference to the folded and unfolded status of the mRNA strand represented by the one-dimensional lattice). From [368].

One such example appears in the context of protein synthesis control, where the lattice of the TASEP then represents a strand of messenger ribonucleic acid (mRNA). Each time a ribosome (a 'particle' in TASEP) moves along the lattice, it synthesizes one protein. One possibility to regulate the amount of synthesized protein is based on the ability of a given region of the mRNA to fold (or to bind with a translation repressor protein), and thus to prevent ribosomes to go further along the mRNA strand. Turci et al [368] have proposed to model this phenomenon by the following model based on a single lane TASEP. The rules are those of the original TASEP, except for one site $s$ (or one region of the lattice) which can be open or closed (see Fig. 16). When site $s$ is closed, particles cannot hop on it, and pile up in a waiting line until site $s$ opens. The opening and closing of site $s$ occur with fixed rates. However, an extra condition for closing site $s$ is that it must not be occupied. This condition couples the dynamics of the lattice with the dynamics of particles and leads to a rich phenomenology, parts of which are still under study. 


\subsubsection{Alternating flows in multi-lane TASEPs}

Some other models have been proposed in which the status of a portion of the lattice which can be open or closed has also a dynamics coupled with the occupation by particles.

A situation encountered in various systems is that oppositely moving particles share a common bottleneck, which is too narrow to allow for crossing. The bottleneck is thus used alternatively by the two types of particles.

Such a model was first proposed in the frame of bidirectional pedestrian flows [171], to model the flow oscillations that are observed at a bottleneck (door or small corridor). But it can also be relevant to model biological phenomena, such as bidirectional molecular traffic across nuclear pores [185].

In the bottleneck model [171] depicted in Fig. 17, two types of particles move respectively towards the right or towards the left. Outside the bottleneck, particles of different types move on different tracks and do not interact. Inside the bottleneck, only one species can go through at a given time, as no exchange of particles is allowed. More precisely, a particle of a given type can enter the bottleneck only if there is no particle of the other type inside the bottleneck.

This constraint is non local and assumes that the particles that are about to enter the bottleneck have a knowledge of the occupation status of the bottleneck. For pedestrians, this can be obtained by visual control. In biological systems, a conformational or chemical change could occur somehow.

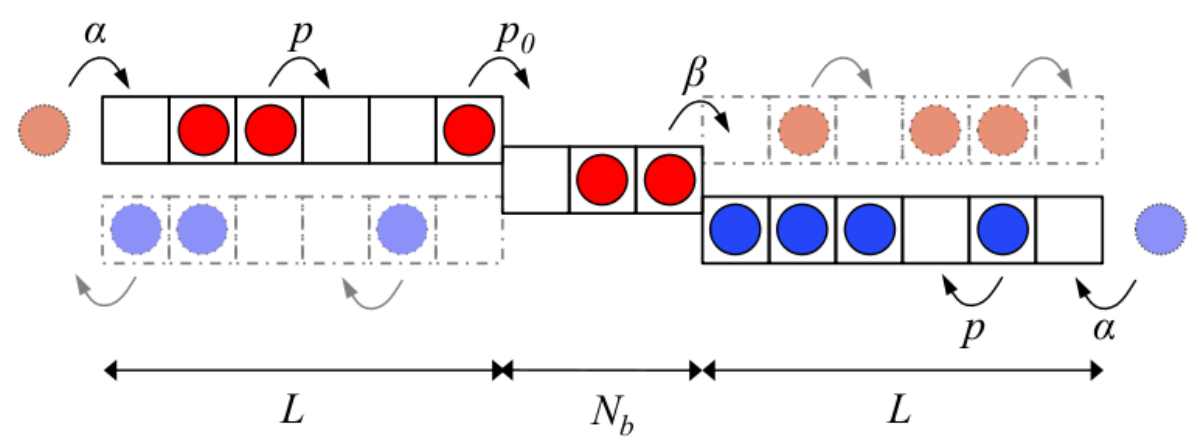

Figure 17: Two TASEP systems interact through the sharing of a common bottleneck. Red particles (upper lane) and blue particles (lower lane) move in opposite directions and can enter the bottleneck of length $N_{b}$ only if there is no particle of the opposite type. From [171].

It has been shown that the dynamics of such a model is driven by fluctuations in the bottleneck and by the transient states that take place at each reversal of the flow in the bottleneck [171]. From the point of view of non-equilibrium systems, it is interesting to have a model that gives macroscopic signatures from local fluctuations.

Another example of on-off gate driven by particles was given in [128], to describe an urban trafic network with "intelligent" trafic lights. There also, the coupling of the trafic lights dynamics with the occupation of the network yields a non trivial phenomenology.

Many features of these dynamical gates whose dynamics depend on particle positions are still not fully understood and raise interesting fundamental questions, with related applications to real systems.

\subsection{Multi-lane exclusion processes}

In the cell, microtubules do not offer only one but several parallel tracks (protofilaments). Here we explore the consequences of having multiple tracks on the TASEP characteristics.

When multilane systems are considered, different behaviors can be observed depending on the type of coupling between the lanes, whether all particles move in the same direction or not, etc.

\subsubsection{Multi-lane interactions without lane changes}

As a simplified case of multi-lane traffic, we consider here two parallel TASEP lanes, labeled A and B in Fig. 18. If there are no interactions between the lanes, the system is trivial and reduces to two independent TASEPs. In this section, we shall consider the case where particles on different lanes can interact. These interactions can be thought for example as steric interactions between cargos transported by motors, that create some friction when they pass each other. Throughout 


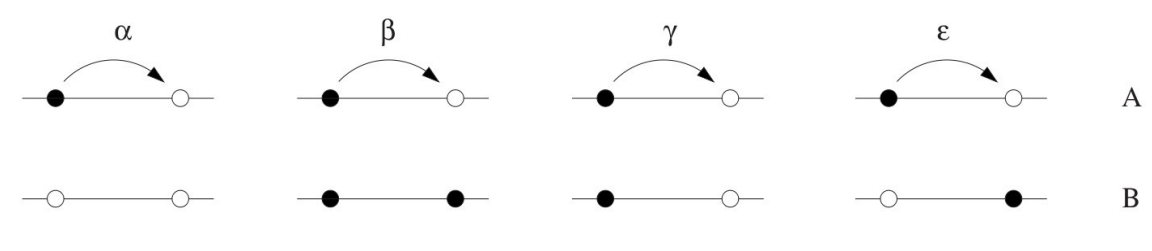

Figure 18: Schematic representation of the hopping rules on lane A. The hopping rates depend on the occupation of lane B. From [292].

this section, particles cannot change lane. The case of particle exchanges between the lanes will be considered in the next section 5.2.

In [220], particles on each lane move in opposite directions. The interaction between the lanes is such that the hopping rate of a given particle is reduced by a fixed factor if there is a particle of the other species on the site next to its target site. This corresponds to the case $\alpha=\gamma>\beta=\epsilon$ in Fig. 18. In the special case where one lane is occupied by cars and the other one by oppositely going trucks (trucks' hopping rates are obtained from the cars' ones by dividing by a fixed factor), it is shown that the interlane interaction can result into the formation of bound states between the trucks, i.e. a truck-truck interaction mediated by the cars is obtained.

Another case where particles go in the same direction on both lanes was considered in [293], with the choice $\alpha=\beta=$ $\gamma=1$ and $\epsilon<1$. For open boundary conditions, this model exhibits a new phase where the system oscillates between symmetry breaking phases [293], for which one lane has a much higher density than the other one. This behavior results from the existence of fast and slow processes.

Eventually, for the case $\alpha=1$ and $\gamma=\epsilon=(1+\beta) / 2$, a domain wall description of the two-lane system shows that, while for a single lane the relaxation towards the stationary state is obtained through only one reflection of the shock from either of the boundaries, multiple reflections of shocks, which now come in pairs [294], are necessary in the two-lane system [292].

\subsubsection{Multi-lane uni-directional transport with bulk lane changes}

In this section, we consider only particles going in the same direction, on a system of parallel tracks. When several lanes are offered for transport, in many systems it is possible that particles change lane. This is for example possible for dynein motors which are able to change the protofilament when walking along microtubules. Lane changes can be simply modeled by rates $\omega^{\downarrow}$ and $\omega^{\uparrow}$ at which a particle hops to the neighbor site on the lower or upper lane (fig. 19).

In order to analyze the effect of lane changes in terms of a mean-field approach, a set of equations has been introduced, that expresses mass conservation. If we call $j^{\uparrow}\left(i+\frac{1}{2}\right)$ (resp. $\left.j^{\downarrow}\left(i+\frac{1}{2}\right)\right)$ the horizontal flux between site $i$ and site $i+1$ on the upper lane (resp. lower lane), and $h^{\downarrow}(i)$ (resp. $h^{\uparrow}(i)$ ) the vertical flux at position $i$ from the upper to the lower (resp. from the lower to the upper) lane, then mass conservation can be expressed as

$$
\begin{aligned}
\rho^{\uparrow}(i, t+d t)-\rho^{\uparrow}(i, t) & =\left[j^{\uparrow}\left(i-\frac{1}{2}, t\right)-j^{\uparrow}\left(i+\frac{1}{2}, t\right)+h^{\uparrow}(i, t)-h^{\downarrow}(i, t)\right] d t \\
\rho^{\downarrow}(i, t+d t)-\rho^{\downarrow}(i, t) & =\left[j^{\downarrow}\left(i-\frac{1}{2}, t\right)-j^{\downarrow}\left(i+\frac{1}{2}, t\right)+h^{\downarrow}(i, t)-h^{\uparrow}(i, t)\right] d t
\end{aligned}
$$

Replacing the fluxes by their mean-field expressions

$$
\begin{aligned}
j^{\uparrow}\left(i+\frac{1}{2}\right) & =p^{\uparrow} \rho^{\uparrow}(i)\left[1-\rho^{\uparrow}(i+1)\right] \\
j^{\downarrow}\left(i+\frac{1}{2}\right) & =p^{\downarrow} \rho^{\downarrow}(i)\left[1-\rho^{\downarrow}(i+1)\right] \\
h^{\uparrow}(i) & =\omega^{\uparrow} \rho^{\downarrow}(i)\left[1-\rho^{\uparrow}(i)\right] \\
h^{\downarrow}(i) & =\omega^{\downarrow} \rho^{\uparrow}(i)\left[1-\rho^{\downarrow}(i)\right]
\end{aligned}
$$




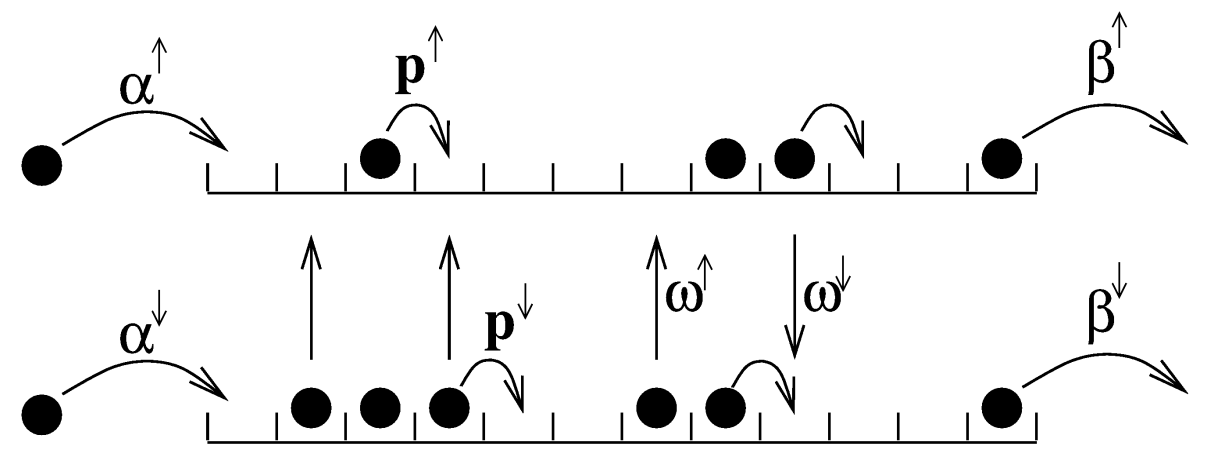

Figure 19: Schematic representation of the rules coupling two parallel TASEP lanes. The exchange rates for the bulk coupling are called here $\omega^{\downarrow}$ and $\omega^{\uparrow}$. In the text, we assume that $p^{\uparrow}=p^{\downarrow}=1$, though a generalization to arbitrary values is straightforward. From [326].

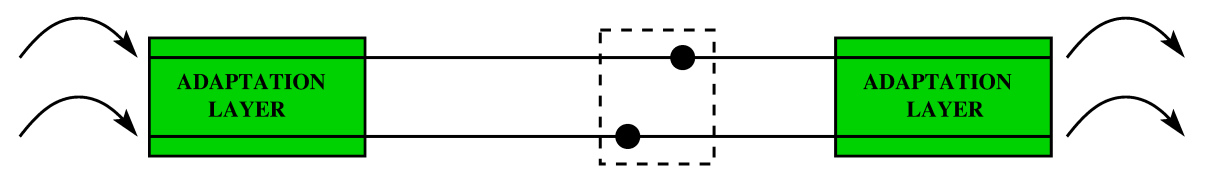

Figure 20: Typical structure of a two-lane system with strong coupling. Non-adapted boundary conditions create adaptation layers on both sides. In the bulk, pairs of shocks are formed and move as a whole through the system. Then the net vertical flux is zero everywhere except in adaptation layers and inside the shock pairs. From [326].

yields, after a change of variables $x=i / L$ and an expansion in the limit $L \gg 1$, the following equations for the density profiles:

$$
\begin{aligned}
& \partial_{t} \rho^{\uparrow}=-\frac{1}{L} p^{\uparrow}\left(1-2 \rho^{\uparrow}\right) \partial_{x} \rho^{\uparrow}+\omega^{\uparrow} \rho^{\downarrow}\left(1-\rho^{\uparrow}\right)-\omega^{\downarrow} \rho^{\uparrow}\left(1-\rho^{\downarrow}\right) \\
& \partial_{t} \rho^{\downarrow}=-\frac{1}{L} p^{\downarrow}\left(1-2 \rho^{\downarrow}\right) \partial_{x} \rho^{\downarrow}+\omega^{\downarrow} \rho^{\uparrow}\left(1-\rho^{\downarrow}\right)-\omega^{\uparrow} \rho^{\downarrow}\left(1-\rho^{\uparrow}\right)
\end{aligned}
$$

Note that the two rightmost terms correspond to the the net vertical flux $h^{\text {net }} \equiv h^{\uparrow}-h^{\downarrow}$. In these equations, the dominating terms will be different depending on the scaling of the coupling rates $\omega^{\downarrow}$ and $\omega^{\uparrow}$ (to simplify the discussion we shall assume in the following that $p^{\uparrow}=p^{\downarrow}=1$ ).

Strong Coupling. If the exchange rates are of the same order as the hopping rates, the coupling is said to be strong. This is for example the case if one considers highway traffic or the dynamics of dynein in a crowded environment. If the inand output rates are the same on both lanes, both lanes behave in a similar way, shocks on both lanes are coupled [255] and the phase diagram can be derived quite easily [296]. The dynamical characteristics of coupled lanes becomes more complex when the lanes have different initial states, and different in- and output rates [326].

We shall first discuss the transient occuring when two initially independent TASEP lanes with different in- and output rates are coupled at $t>0$ according to the rules of Fig. 19. From the mean-field equations (31-32), we see that when the coupling rates are of order $O(1)$, strong lane exchanges can take place over time scales of order 1 . Indeed, this is what is observed in direct simulations: If the system is initialized with arbitrary constant densities, very intense lane exchanges in the bulk lead within a few time steps to a local balance between the lanes. When the densities on the two lanes are such that the net flux is vanishing, we consider the lanes to be in an adapted state. For arbitrary in- and output rates, some adaptation layers localized near the boundaries interpolate between the non-adapted densities imposed by the reservoirs to the bulk densities which balance the vertical fluxes (Fig. 20). After the initial adaptation, the net vertical flux vanishes almost everywhere, except in shock regions and in adaptation layers.

If a shock - or domain wall - exists initially on one lane in the bulk, after the initial adaptation stage another shock will be formed on the other lane, in the vicinity of the first one. These two shocks form a pair separated by a transition region within which a persistent vertical net flux of particles exists (see Fig. 21). The motion of the two shocks is strongly 


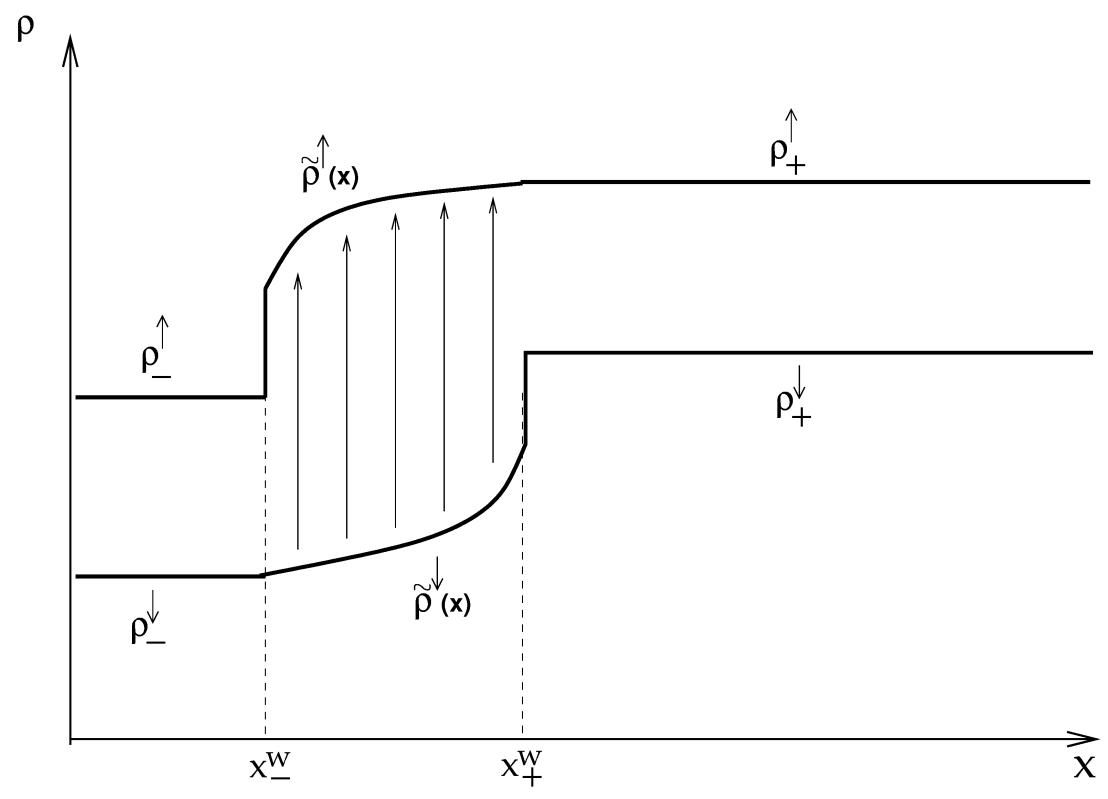

Figure 21: Schematic representation of the structure of a pair of walls. The walls are represented a real discontinuities separated by a non-constant density profile on the two lanes. From [326].

correlated, since their distance is controlled by the amplitude of the local vertical flux. Depending on the initial condition, several such shock pairs can be formed. On much longer time scales, these pairs perform biased random walks, meet and merge, until a unique shock-pair remains in the system.

In the long time limit, a stationary state is reached. In most cases, the remaining shock pair localizes itself near a boundary, as in the one lane case. Here however, the inner structure of the shock pair interfere with the adaptation layer so that the stationary state is more difficult to predict. The characteristics of the stationary state, and in particular the phase diagram, were studied first in [155] in the case of very asymetric lane changes, and more recently obtained for more general rules from a stability analysis of the mean-field profiles [119]. The case of back-stepping was also considered [119, 367].

Weak Coupling. Weak coupling refers to the case where the exchange rates (and the net current between lanes) scale as $1 / L$ and was in particular studied in [304, 306, 305]. It has been argued that weak coupling could be relevant for example for kinesin based intracellular transport: kinesins do not usually side-step, but lane changes could be carried out via an intermediate detachment of the molecular motors [304] or under the effect of lateral loads.

In the case of weak coupling, all terms in the mean-field equations are of the same order. Now the net vertical flux is not necessarily vanishing and the density profile varies smoothly. Contrarily to strong coupling for which shock positions on both lanes are strongly correlated, in the case of weak coupling, shocks are only loosely coupled. Actually, in this scaling, it is as if the shock pairs found for strong coupling were now separated by a distance of the order of the systems size. Thus the inner structure of a shock pair shown in Fig. 21 in the case of strong coupling is the analog of the density profiles in the case of weak coupling.

If boundaries are imposing different fluxes, the shocks will be localized at the boundaries in the stationary state. If boundary reservoirs are imposing similar fluxes, but different densities, then there is a need to match the solutions coming from both ends, and shocks will form in the bulk. While for the single lane TASEP, shocks in this case are not localized and explore the whole system according to a symmetric random walk, here the shocks can be localized in the bulk if there are some adaptation layers. Indeed, while adaptation layers were always of finite size in the case of strong coupling (see Fig. 20), they may now invade the whole system.

Though these approaches are useful to understand the role of lane changes, note that in the case of molecular motors, an analysis taking into account the non-conservation of mass on the filament would be more appropriate, as developped 
in section 6.2.

\subsubsection{Multi-lane bi-directional transport with bulk lane changes}

While in the previous section the lanes could represent protofilaments on the same MT, it is also possible to have motor exchanges between filaments (MTs or actin) oriented in opposite directions. We shall thus consider in this section the coupling of two TASEP lanes, where particles are going to the right on one lane and to the left on the other lane. This was done in [174] in the case of weak coupling. As mentioned in [174], due to the particle/hole symmetry, one could actually replace the holes on one lane into particles, to have again uni-directional motion, but the interaction between the lanes would then be that particles would be created or annihilated in pairs.

Several types of boundary conditions can be considered. For example, while [174] deals with open boundary conditions, the case of one open and one closed end is studied in [22] with a focus on the length of the queuing line that forms near the dead end.

\subsection{Modelling inhomogeneities}

Motor-driven intracellular transport is usually carried out on filament networks which are embedded in a crowded environment. Although the bulk structure of the filaments is surprisingly regular several mechanisms exist which influence the local transport properties of the chain. The environment, more or less crowded, can change locally the transport capacity on a given MT. Besides, several kinds of proteins can bind to the MT, a prominent example detailed in the next subsection being tau proteins. Tubulin itself can be locally modified: The cell can produce different isotopes of tubulin, and various enzymes can induce some modifications of tubulin after polymerization [168]. These modifications could have deep functional implications, and even lead to "functionally distinct microtubule types" [167].

The motors also can see their properties deeply modified by external factor, e.g. dynactin on dynein. Besides, the large variety of cargos that need to be transported provides a very inhomogeneous crowd of moving objects.

It is therefore interesting to discuss the impact of inhomogeneities on transport. Actually the impact of disorder on transport has already be largely studied in the context of the simple TASEP. Two families of disorder have been considered: Link or site-wise disorder, which would model the inhomogeneities of the tracks, and particle-wise disorder, corresponding to the inhomogeneities in cargo types. Particle-associated disorder can be modelled by assigning different hopping rates to different particles, while for site-associated disorder, the hopping rates $p_{i}$ are fixed but not equal for all sites in the system.

In case of periodic boundary conditions the TASEP with particle-wise disorder can be exactly solved [115] by introducing distance variables $[115,211]$ instead of particle positions. This mapping relates the TASEP to the zero-range process which is exactly solvable even in the presence of disorder. The mapping is more difficult to exploit in the case of open boundary conditions, as the number of particles is not conserved.

When particles do not move at the same speed, they may pass each other, taking advantage of the possibility to use parallel tracks. K. Mallick solved a one-lane TASEP with one single slow particle that could be passed by fast particles with some probability less than 1 [240]. As expected, jamming can occur behind the slow particle. A two-lane TASEP model with particle-wise disorder was proposed in [202] and showed numerically the strong tendency to form platoons behind slow particles. However no exact solution has been found even for periodic boundary conditions. Some exact results have been obtained for similar systems in continuous space and time (kinetic models), first for a one lane model in which passing is allowed with a certain rate [35], or for a two lane model with bidirectional trafic, under a mean field coupling assumption [17]. Back in the frame of TASEP-based models, some recent analytical results were obtained for a one-lane model where particles have the same speed, but have different priorities for passing each other [124, 117, 18, 19] - a feature that results into different effective velocities.

By contrast with the particle-wise disorder TASEP, the TASEP with site-disorder is not exactly solvable even in the stationary state for periodic boundary conditions, except in some quite special cases (for example for a single site disorder with alternated parallel update [333]). However, in recent years, a number of works have approached the sitewise disordered TASEP by numerical methods and analytical approximations. Computer simulations have shown that even a single defect, i.e. a site with reduced hopping rate may introduce a macroscopic high and low density domain to the stationary state of the TASEP with periodic boundary conditions [169].

Next to a single defect site also more than one defect site in the system has been considered. In [366] the site dependent hopping rates are independently chosen from a random distribution. As in the case of a single defect, disorder can lead to macroscopically nonhomogeneous states, which are observed for densities in a finite range around half filling, while at 
low and high densities only local variations of the density are observed. The self-organization into a macroscopic high and low density domain is generated by a stretch of sites carrying small hopping rates. Note that this stretch does not automatically include the site with the lowest hopping rate if this site surrounded by fast links. This is in contrast to particle disorder where the phase separated states in uni-directional systems are determined by the slowest particle.

To come closer to motor dynamics, TASEP has to be supplemented with Langmuir kinetics. In the case of infinite diffusion in the surrounding cytoplasm, as considered in chapter 6.2, the influence of a single stepping defect was studied in [286] and for multiple stepping defects in [148]. While the phases of the defect-free system still persist, new states arise in which the system is subdivided into parts by the defects, leading to phases which are characterized by the coexistence of different domains.

In a generic way, stepping defects and unbinding defects modify the properties of single motors, as the run-length along the filament or the velocity. A run-length reduction due to defects can affect the global motor current in different ways: In a system with periodic boundary conditions, the current is negatively affected by stepping defects but may increase for unbinding defects at high enough motor densities. This is possible due to the reduction of the density of bound motors on the filament [70], which reduces the tendency to form jams on the filament. The model of section 7.3.1 with randomly deleted MT sites [107] can also be seen as an example of such a mechanism. The use of dynamic unbinding defects should actually be even more efficient than static ones in order to reduce the tendency to form jams.

Binding defects on the other hand do not affect collective motion of particles much if not present at almost all sites $[152,70]$.

The consequences of site-wise defects in the models of this section have been studied for particles hopping only in one direction, i.e. representing motors. If they were representing cargos carried by teams of molecular motors, bidirectional motion should be including. In this case, site-disorder might serve to regulate the motion of individual cargos as hypothesized in [97].

\section{Many-particle models for uni-directional stochastic transport: The impact of particle reservoirs}

In this chapter we discuss the impact of different realizations of particle reservoirs on the bulk properties of the system. The importance of particle reservoirs has already been exemplified for the TASEP with open boundary conditions, where bulk phases are selected by the capacity of the boundary reservoirs. In the context of intracellular transport, bulk reservoirs may also be considered. Besides, spatial confinement and limited capacities of the particle reservoirs may have strong impact on the system's behavior.

\subsection{The TASEP with Finite Resources}

The TASEP with open boundary conditions is coupled to infinite boundary reservoirs, which impose constant inand output rates of the particles. This situation might be realized in in vitro experiments, where the occupation of the cytoskeletal filaments has typically little impact on the concentration of molecular motors in solution. In cells, however, the number of transported objects is limited, and this has to be considered in theoretical approaches as well.

In this section, in order to single out the effect of finite resources, we discuss their impact on the standard TASEP with open boundaries, which are then realized by explicit particle reservoirs rather than by in- and output rates. This has first been done in a different context ${ }^{14}$ by Ha and den Nijs [153] who fixed the total number of particles $N_{\text {tot }}$ in the system and expressed it as

$$
N_{\text {tot }}=N_{\mathrm{b}}+N_{\mathrm{u}}
$$

where $N_{\mathrm{b}}$ is the number of particles on the lattice and $N_{\mathrm{u}}$ the number of particles outside the lattice in the boundary reservoirs (unbound particles). The effective entrance rate $\alpha_{\text {eff }}$ depends on the number of particles in the reservoir:

$$
\alpha_{\mathrm{eff}}\left(N_{\mathrm{u}}\right)=\alpha f\left(N_{\mathrm{u}}\right)
$$

The function $f\left(N_{\mathrm{u}}\right)$ satisfies the conditions $f(0)=0, f(\infty)=1$ and is monotonously increasing in order to respect the availability of particles in the reservoir. In [153], the case $\beta=1$ was considered, whereas Adams et al. [2] had a more

\footnotetext{
${ }^{14} \mathrm{Ha}$ and den Nijs actually motivated their model with the garage parking problem.
} 


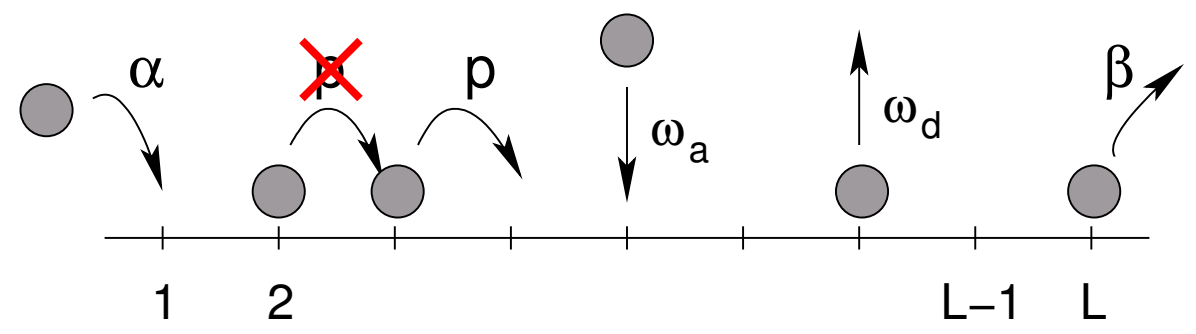

Figure 22: TASEP with Langmuir kinetics. In addition to the usual TASEP hopping rules, particles are also exchanged with a reservoir in the bulk of the system: A particle detaches from the lattice at rate $\omega_{\mathrm{d}}$ and an empty site gets occupied by a particle at rate $\omega_{\mathrm{a}}$.

complete look at the modifications brought about to the whole phase diagram by the finite resources. Finite resources have also been considered with respect to the activity of particles, which is limited by the supply of energy [50].

It is easy to see that for $N_{\text {tot }} \rightarrow \infty$ the standard TASEP as presented in section 4.1 is recovered with $\alpha_{\text {eff }}=\alpha$. Interesting effects are observed if the total number of particles is chosen low enough in order to constrain the number of particles on the lattice and induce feedback mechanisms between the densities on the lattice and in the reservoir. In particular, $\alpha_{\text {eff }}$ can depend on time because the number of particles attached to the lattice can vary. Rather than discussing the particular properties of different finite particle reservoir realizations we will focus on their general impact on the bulk states.

First of all it should be noted, that $f\left(N_{\mathrm{u}}\right)$ can be chosen such that the particle-hole symmetry of the TASEP is broken, which would lead to a modified phase diagram of the system in the stationary state. For the open TASEP with infinite particle reservoirs a phase transition from a low density to a high density phase is observed at $\alpha=\beta(\alpha, \beta<0.5)$. This is also true for systems with finite resources, but the effective entrance rate $\alpha_{\text {eff }}$ has to be considered, while the exit rate $\beta$ is unchanged. Similarly, the transition to the MC phase is reached if $\alpha_{\text {eff }} \geq 1 / 2$ and $\beta \geq 1 / 2$, where the ability to reach a MC phase obviously depends on the total capacity of the boundary reservoirs.

The finite reservoir may not only modify the phase boundaries but also the dynamics of the shock that appear when a jammed and free flow phase coexist. In case of the standard TASEP the shock velocity is independent of its position. This is not always the case for finite particle reservoirs since $\alpha_{\text {eff }}$ depends on the number $N_{\mathrm{b}}$ of bound particles which itself depends on the position of the shock [82]. The shock position is localized via the following mechanism: At the mean position of the shock the condition $\alpha_{\text {eff }}=\beta$ holds and the motion of the shock is unbiased. However, if the shock is displaced towards the entry of the system the value of $\alpha_{\text {eff }}$ is lowered since more particles are in the system at the expense of the reservoir. Similarly $\alpha_{\text {eff }}$ is increasing for displacements of the shock towards the exit. Therefore, this feedback mechanism localizes the shock at its mean position. This mean position can be located anywhere along the filament, in contrast with the case of infinite reservoirs for which shock localization can occur only at the boundaries.

Next to the localization of the shock, other non-trivial effects have been observed for the TASEP with finite boundary reservoirs [2, 82]. We won't discuss this in more detail but switch to models which consider the fact that the runs of molecular motors along the filament have a finite length (smaller than the filament length) due to the possibility to detach - and subsequently re-attach.

\subsection{The TASEP with Langmuir Kinetics}

Molecular motors switch between phases of directed and diffusive motion. During the latter, they are not attached to filament but move randomly through the cytoplasm under the constraints of other cytoskeletal elements. This detachment and attachment of motors to the MT leads to a local non-conservation of particles and current in the bulk and has been considered in the most simple way in a model by Parmeggiani et al. [282] who coupled the open-boundary TASEP of section 4.1 with Langmuir kinetics in the bulk. More precisely, a particle detaches from a lattice site in the bulk at rate $\omega_{\mathrm{d}}$ and an empty site becomes occupied by a particle at rate $\omega_{\mathrm{a}}$ (figure 22). The model exhibits the same type of particlehole symmetry as the TASEP under the additional symmetry operation $\omega_{\mathrm{d}} \leftrightarrow \omega_{\mathrm{a}}$. From a physical point of view the attachment and detachment of particles corresponds to a coupling of the bulk of the system to a grand-canonical reservoir with constant density. Particles within the bulk reservoir have an infinite diffusion rate since a detached particle loses any memory of the site of previous attachment. 
Similarly to what was done in section 5.2 for coupled TASEP lanes, some continuous mean-field equations can be derived from the microscopic equations in the limit of large systems: a change of variable $x=i / L$ and an expansion using the fact that $L \ll 1$ yields

$$
\frac{\partial \rho}{\partial t}=-(1-2 \rho) \frac{1}{L} \frac{\partial \rho}{\partial x}+\omega_{\mathrm{d}}\left[\frac{\omega_{\mathrm{a}}}{\omega_{\mathrm{d}}}-\left(1+\frac{\omega_{\mathrm{a}}}{\omega_{\mathrm{d}}}\right) \rho\right],
$$

where $x=i / L$. The dominating terms in this equation depend on the order of the coupling rates $\omega_{\mathrm{d}}$ and $\omega_{\mathrm{a}}$. In the same way as discussed for two-lane systems, we could consider strong coupling to the bulk reservoir, for which the rates $\omega_{\mathrm{a}}, \omega_{\mathrm{d}}$ are of order one. Then the second term in (35) would dominate for large systems. However, in the case of intracellular transport, it is generally considered that it is the weak coupling in the sense of (36) that is relevant, since the run lengths of processive molecular motors are of the order of the filament length, as mentionned in section 3.1.1 :

$$
\omega_{\mathrm{a}}=\frac{\Omega_{\mathrm{a}}}{L} ; \quad \omega_{\mathrm{d}}=\frac{\Omega_{\mathrm{d}}}{L} ; \quad \text { with } \Omega_{\mathrm{a}}, \Omega_{\mathrm{d}}=\text { const. }
$$

The current between lanes is then of order $1 / L$ and time must be rescaled as $\tau=t / L$, so that all terms in (35) are of the same order [118] :

$$
\frac{\partial \rho}{\partial \tau}=-(1-2 \rho) \frac{\partial \rho}{\partial x}+\Omega_{\mathrm{d}}\left[\frac{\Omega_{\mathrm{a}}}{\Omega_{\mathrm{d}}}-\left(1+\frac{\Omega_{\mathrm{a}}}{\Omega_{\mathrm{d}}}\right) \rho\right],
$$

Stationary states and phase diagram. When searching the stationary state $\partial \rho / \partial \tau=0$ of this model, equation (37) obviously leads to a first-order differential equation. The couplings to the boundary reservoirs on the left and right end of the lattice impose two boundary conditions $\rho(x=0)=\alpha$ and $\rho(x=1)=1-\beta$. In other words, the problem is over-determined. The overall solution [118] consists of the two individual solutions resulting from integration of the differential equation from the left and from the right side of the system. The transition point from one solution to the other can be determined in the framework of kinematic wave theory. If the densities of the two solutions do not match at the point of transition, a shock (or domain wall) occurs which depends on higher orders of $L^{-1}$. Consequently, this shock gets sharper the longer the system is.

In contrast to the TASEP, the shock front does not move within the system, once the system has reached its stationary state. Instead, the shock is driven to the position in the system where the mass current through the shock front is zero (see figure 23), so that localization of the shock occurs [118]. This phenomenon is interesting in so far as no defects on the lattice are implicated and the particle dynamics is homogenous in the bulk of the system. Surprisingly, on the line $\alpha=\beta<1 / 2$, the localization effect persists even if the bulk reservoir density vanishes with increasing system size, i.e., $\omega_{\mathrm{d}}, \omega_{\mathrm{a}} \propto L^{-a}$ with $1 \leq a<2[175]$.

In contrast to the TASEP two macroscopic boundary domains and a bulk domain can be imposed simultaneously in the stationary state. Therefore, the number of qualitatively different density profiles is much higher than for the TASEP and depends strongly on the attachment $\left(\omega_{\mathrm{a}}\right)$ and detachment $\left(\omega_{\mathrm{d}}\right)$ rates $[118,283]$ and the phase diagrams exhibit more different phases than for the TASEP. More precisely, in addition to the three phases already known from the TASEP (see section 4.2.3), there is also a phase corresponding to stationary states with a localized shock in the bulk of the system. Furthermore, there are several states in which two or all three TASEP phases coexist. In these phases, no shock is observed and the partial profiles are continuously connected with a cusp in the density profile. If $\Omega_{\mathrm{a}} \neq \Omega_{\mathrm{d}}$, the maximal-current phase is suppressed since the Langmuir kinetics tries to bring the bulk of the system to a density $\rho_{\text {eq }} \neq 1 / 2$, thus driving it away from the maximal-current density.

Altogether, the Langmuir kinetics brings about some very interesting effects although it is an uncorrelated equilibrium process which would not lead to phase transitions on its own. In combination with a non-equilibrium process, however, the Langmuir kinetics modifies the phase transitions found in the TASEP and leads to qualitatively new behavior.

Similar results are obtained if two parallel lattices are considered, which exchange particles with a bulk reservoir via Langmuir kinetics and are additionally able to exchange particles between filaments [382]. The qualitative behavior also remains unaltered, if particles are considered to be dimers whose two heads individually bind to two neighboring sites and walk in the hand-over-hand fashion described for kinesin in section 3.1.1 [285].

The model also allows for comparison with in vitro experiments, where the concentration of the molecular motors and of ATP can be controlled. The motor concentration in the reservoir is proportional to the attachment rate while 


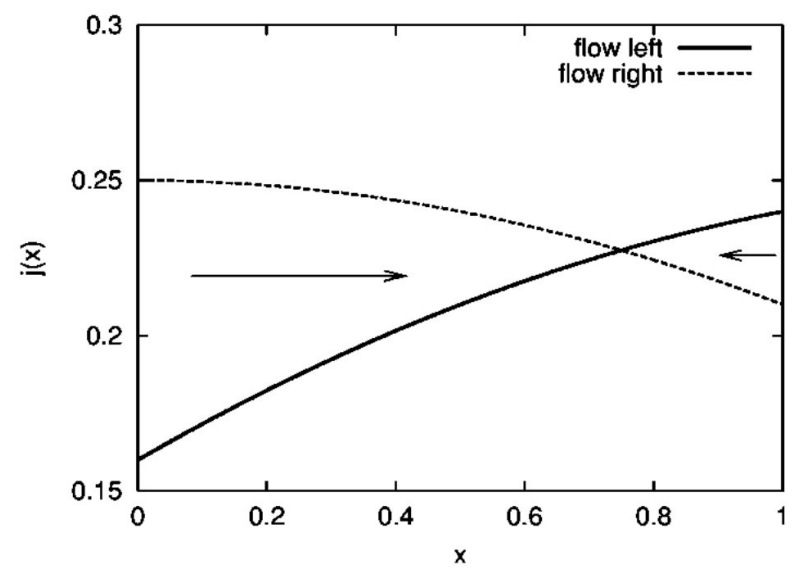

Figure 23: Typical flow profile of the TASEP with Langmuir kinetics in the shock phase. The solid (dashed) line indicates the flow profile induced by the left (right) boundary reservoir. The arrows indicate the bias of the shock. From [118].

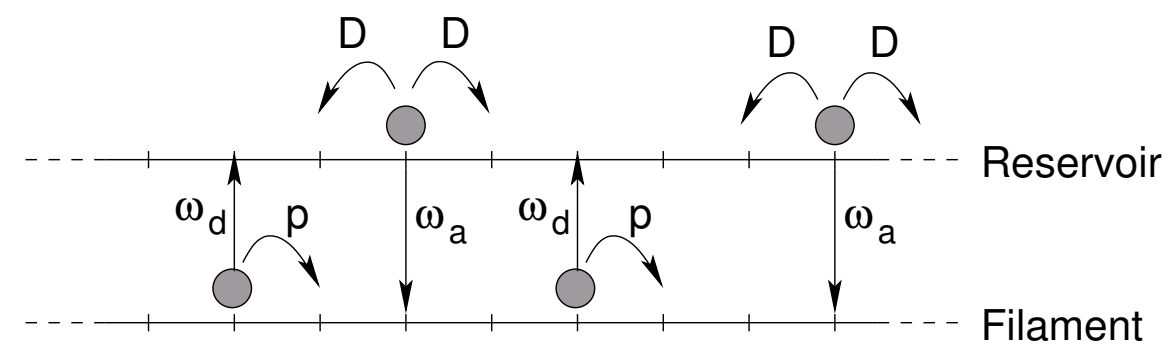

Figure 24: One-species TASEP coupled to a diffusive lane. The model consists of two one-dimensional lattices. The lower lattice represents the filament and serves as track for directed motion of the particles (represented as disks) which interact via hard-core exclusion on this lane. The upper lane is a diffusive environment with unbiased motion and without any interaction between particles. As a result, one site of the diffusive lane can be occupied by several particles at the same time.

the ATP-concentration has strong influence on the stepping rate of the motor proteins (see [275] and our discussion in section 3.2.2). .

In order to improve the realism of the theoretical model the substructure of the filaments has been taken into account. In [75], Chowdhury et al extend the model to several parallel lanes, allowing for lane-changes. These lanes could represent the 13 protofilaments that compose the MT. Their model includes not only Langmuir kinetics but also a minimal description of the mechanochemical cycle responsible for motor stepping (see section 3.2.2). They show that including lane changes may increase or decrease the flux per lane, depending on the motor concentration and the rate of hydrolysis of ATP. They suggest that this could be checked by in vitro experiments.

\subsection{Explicit Realizations of Particle Reservoirs}

As a first step toward a realistic description of the particle reservoir, one may represent the particle reservoir by an additional lane, parallel to the filament, as sketched on figure 24. On the diffusive lane, there is no exclusion principle ${ }^{15}$, and particles diffuse freely by hopping with equal rates to their right or to their left. On the filament lane, particles are subject to directed motion and exclusion. This model is relevant for filaments that are embedded in a small volume such that the motors do not drift too far away from the filament. Moreover the density of motors should be low since

\footnotetext{
${ }^{15}$ Actually the diffusive lane represents the whole volume around the filament, and thus several particles can be present at the same time at a given position.
} 


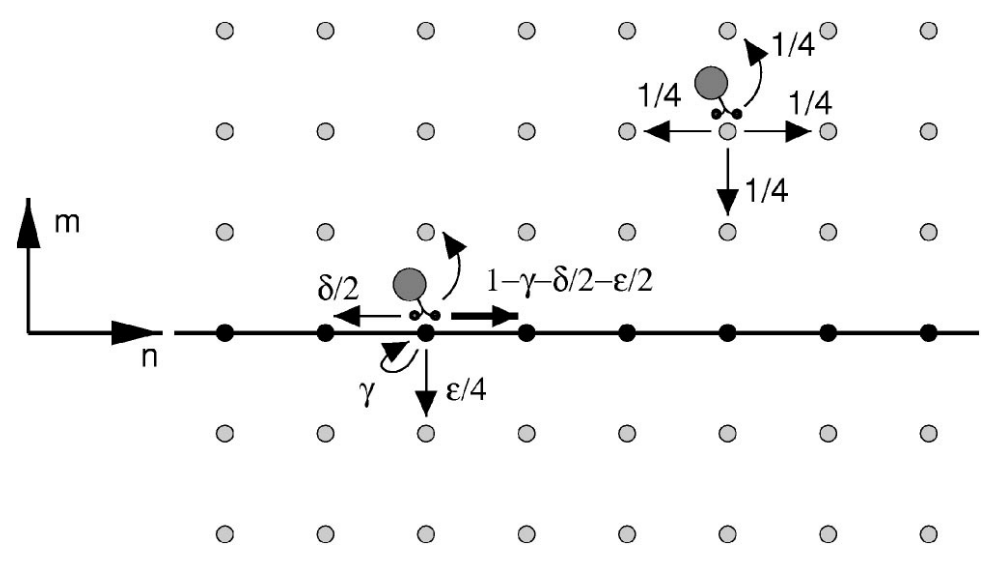

Figure 25: Model of [274] with explicit consideration of the bulk reservoir. Particles hop between neighboring nodes on a cubic lattice, where the filament is represented as a line of black nodes and the bulk reservoir by grey nodes. Hopping within the bulk reservoir is unbiased, but hopping to and away from filament sites depend on the attachment and detachment rates. Please note that the notation of the rates in this figure does not correspond to the notation in the remaining of this review paper. From [274].

exclusion effects are not considered in the diffusive lane. In vitro, this confinement could be achieved in micro-channels or nanotubes. We shall discuss this confinement assumption for an in vivo situation (axonal transport) in section 7.1.3.

The properties of the stationary state for such a two-lane model, and in particular the density profile, have been calculated by M.R. Evans et al in [119].

Lipowsky et al. [229] have introduced a many particle model for motor-driven transport which describes not only the motion of the molecular motors on the filament but also in the reservoir. The model is defined in a three-dimensional geometry with discretized space. Particles hop between nodes of a cubic lattice with spacing $l$ which corresponds to the step size of the molecular motor on a filament, e.g., $8 \mathrm{~nm}$ for kinesins on MTs. As in the previous models, the filament is represented by a linear arrangement of lattice nodes while all other nodes represent the surrounding volume (the cytoplasm in a cell, the solution for an in vitro experiment) where motors undergo diffusion. The filament nodes differ from the nonfilament nodes by the hopping rules of the particles. Filament-bound particles undergo directed motion. They hop forward with rate $p$ provided the target site is empty and never step back. Unbound particles undergo an unbiased random walk on a discrete three-dimensional lattice while interacting with each other via hard-core exclusion (figure 25). Because of the cubic lattice structure, every filament site can be reached from four reservoir nodes. The ratio of the hopping rates from these four sites to the filament and back, i.e., the attachment and detachment rates, determines the affinity of a particle to the filament. The model enforces the conservation of the number of particles and the hopping between reservoir nodes determines the strength of diffusion.

This model includes more features of intracellular transport than the previously presented models by explicitly considering a three-dimensional geometry within which a filament is placed, so that different cellular or experimental environments can be modeled. Lipowsky et al. [229] have tested the outcome of different geometries while estimating the rates from real experiments. This should allow a more direct comparison with experiments.

The explicit consideration of the particle reservoir shows that the attachment and detachment rates are not constant since strong concentration gradients of the particles in reservoirs are observed. The actual distribution of the particles depends strongly on the chosen reservoir geometry [273, 274, 229, 200, 196, 78].

In order to illustrate the influence of the system's geometry we briefly discuss the case of a mixed choice of an open boundary at the minus-end of the filament and a closed boundary at the plus-end [265]. This choice mimics for example the situation in an axon (see section 7.1) more accurately where the closed end represents the synapse and the open end the connection to the cell body which provides the axon with a constant density of molecular motors. Using this geometry, Müller et al. showed that the density on the filament in the stationary state either approaches zero or one far from the open end, i.e., either the long tube is empty or all transport is blocked due to a jam extending through the whole system. The transition between these two states obviously depends on the strength of the diffusion which drives particles back to the open end against the preferential motion of particles on the filament. Müller et al. concluded in the article that additional 
regulatory mechanisms are necessary in order to maintain efficient transport in long tube-like geometries as the axon at intermediate densities.

\subsection{Enhanced diffusion on filament networks}

In this section we consider the case where multiple filaments can serve as tracks for the motors. Klumpp and Lipowsky used the framework of the three-dimensional lattice gas to show that certain arrangements of filaments lead to non-directed diffusive movements which can be used for transport over large distances and exhibit very large diffusion coefficients compared to passive diffusion in the absence of filaments [198]. This diffusive motion is driven by phases of directed transport along individual MTs and is thus dependent on the active stepping of individual motion. Klumpp and Lipowsky therefore refer to this phenomenon by the term active diffusion as the overall motion is undirected due to the uncoordinated attachment to filaments of different orientation. They show this type of motion not to depend on the diffusion coefficients in the cytoplasm surrounding the filaments.

Klumpp and Lipowsky discuss the relevance of this enhanced diffusion for several technical applications, but it may also play a role in dendritic transport. Dendrites are very elongated with a parallel MT network. But in contrast to axons, dendritic MTs are not uniformly oriented so that the structure of the MT network might indeed enhance the diffusion of intracellular cargo. However, the same is not true for axons that will be considered in the next section 7.1, and which have an almost uniformly oriented MT network.

\subsection{Continuous models}

We have seen that in the frame of a mean-field approximation, it is usually possible to derive some continuous equations as (37) from the microscopic dynamics. But it is not mandatory to build continuous equations from microscopic models. Some authors have proposed to describe intracellular transport directly at the level of reaction-diffusion-transport continuous equations.

For example, Smith and Simmons [348] have written a set of differential equations for a multi-lane system. They distinguish some lanes with oriented motion (filaments) and a lane for simple diffusion. Only one type of motor was considered, moving on either on a fully polarized network, or on bi-polar filament networks. The latter case would correspond for example to myosin $\mathrm{V}$ on an actin network.

We shall not describe further this class of models, as we are interested in stochastic models in this review. One advantage of stochastic transport models is that it is often possible to relate the model parameters directly to experimental observation.

\subsection{Membrane tubes}

In this section we address a phenomenon quite different from the intracellular transport mechanisms described above, namely the formation of membrane tubes, i.e. the formation of one-dimensional cylindrical objects that are pulled or pushed out of the cell (or organelle) body. This kind of process is for example of great importance for several biological system including the growth of axons out of the soma of nerve cells. Another example is the tubulation of Golgi membranes [341] which is known to be also driven by MT-based motors.

A cooperative effort is required from molecular motors to pull a membrane nanotube from a vesicle. In vitro experiments have shown that if a vesicle is coated with kinesin and then brought near a MT, the kinesins were able to pull a nanotube [315]. This phenomenon has been modeled with a two-lane exclusion process [63, 359]: the particles represent the kinesins, which all have their bounding domain permanently attached to the nanotube. Kinesins which are also bound to the MT, step on it with a constant rate $p$, always in the same direction. Kinesins may detach from the MT and then perform a diffusive motion on the other lane, without any exclusion rule. They become bound again to the MT with a certain attachment rate. Up to this level, the model is strictly equivalent to the model for uni-directional intracellular transport with finite diffusion described in Fig. 24.

What makes this model specific for pulling nanotubes is that the nanotube as a varying length. Similarly, at the end representing the tip of the nanotube, the two lanes of the model may extend and retract with rates $v^{+}$and $v^{-}$. Tailleur, Evans and Kafri [359] propose a mean-field approach that predict reentrant phase transitions, as a result of the competition of the densities imposed by the two ends of the tube and of the dynamics of the shock that separates these densities. They indicate a certain number of signatures of their theory that could be experimentally tested.

This model gives a first example of coupling between the dynamics of the underlying track and transport. We shall see other examples in the next chapter. 


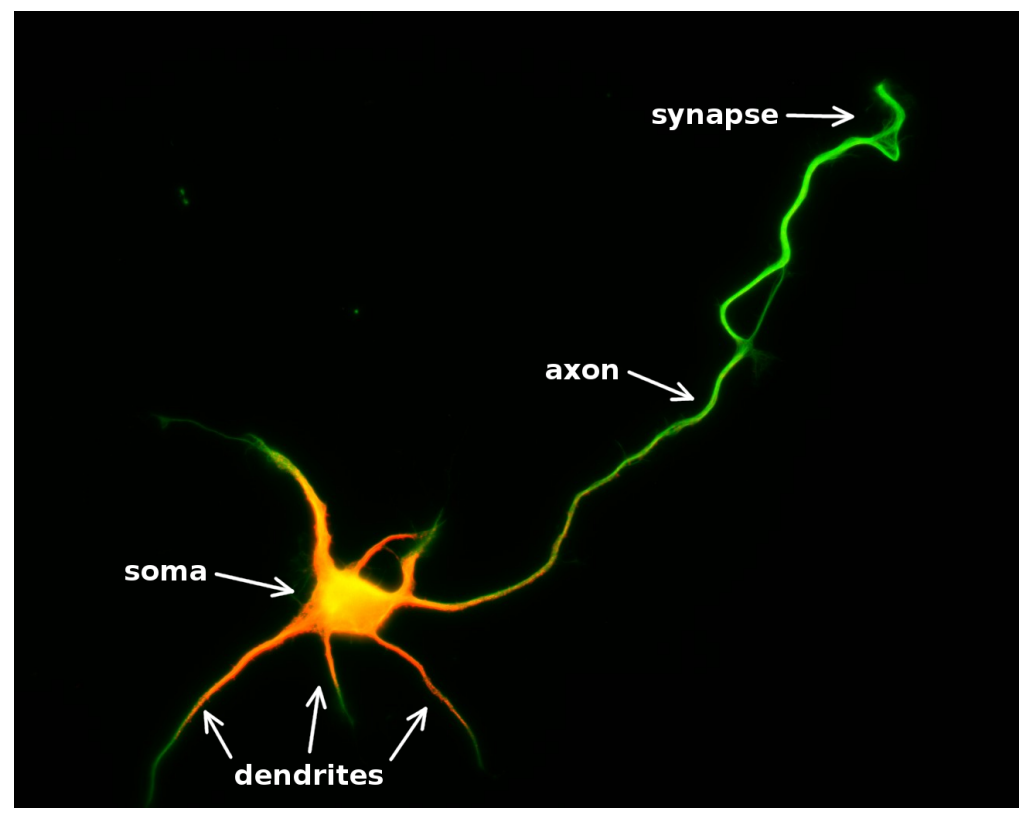

Figure 26: Cultured hippocampal neuron after 2 days in vitro. One can distinguish the axon, the dendrites and the soma. Modified from [51].

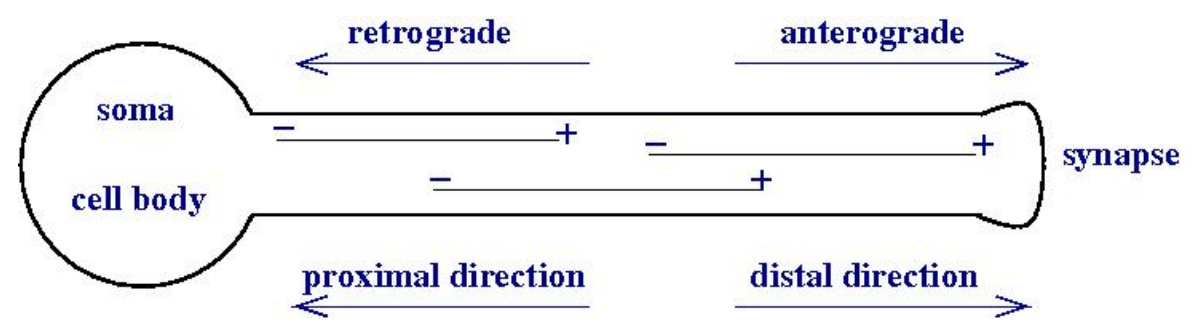

Figure 27: Schematic representation of a neuron and of the directions associated with various terms used to describe axonal transport.

\section{Bi-directional transport and spatial confinement}

Throughout this section, we shall refer more precisely to axonal transport, as it brings some specific questions and gives a precise frame for our discussion. However, it must be kept in mind that, though the axon is the biological system that motivated the present discussion, a fully realistic description of axonal transport is still lacking, and this section will be followed by a list of open questions (section 8) that still need further investigations, both on the point of view of experiments and models. We shall present in this section attempts to understand the basic phenomena that could underly axonal transport and more generally MT-based transport. But first we shall describe the biological system under consideration, namely the axon.

\subsection{Biological description of the axon}

The axon is one of the neurites of a neuron and as such connecting the distant synapse with the cell body or soma (figure 26). In vertebrates, axons are among the longest structures at the level of individual cells with lengths of up to $1 \mathrm{~m}$ in humans while having thicknesses typically not exceeding the micrometer scale [352, 141]. The axon therefore presents a quasi-one-dimensional system within which intracellular cargos are bidirectionally transported from the soma to the synapse and vice versa [259]. 


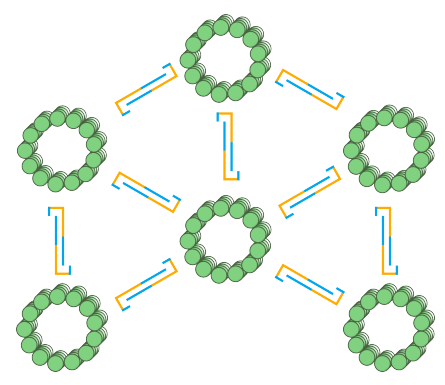

Figure 28: MT spacing by MAPs in axons: Sketch of the cross-section of an axon. Tau dimers (blue and orange) bundle MTs (green rings) and induce a hexagonal structure of the axonal MT network.

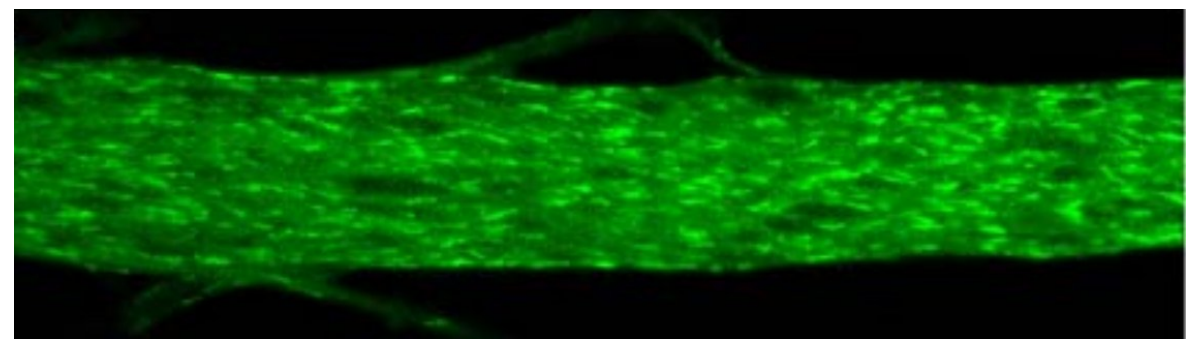

Figure 29: Confocal microscopy imaging of the dynamics of MT plus-ends in the axon of cultured Aplysia neurons. Some fluorescent plus-end-tracking protein allows to mark the plus-ends of MT that are polymerizing. The resulting 'comet tails' show the orientation of the MT, with a majority of MT plus-ends pointing towards the distal tip of the axon. The width of the axon is approximately 10 microns. The video provided in the supplementary materials of [343] (Movie S1A) shows the very dynamical behavior of MT tips. Reprinted from [343].

\subsubsection{Structure of the axonal microtubule network}

The axonal MT network is highly polarized and consists of many MTs of different lengths. The MTs are longitudinally arranged and a large majority point with their plus-end in distal direction, i.e. away from the soma [29, 183]. Transport towards the synapse or the soma have different characteristics, obtained in particular through this high polarisation of the MT network, and some vocabulary has been developped to distinguish among these two transport directions (see sketch in Fig. 27 for a summary of the terminology). It seems for example that organelles that move towards the distal end are on average smaller but more numerous than those moving towards the soma [259].

Individual MTs may be as long as $\sim 100 \mu \mathrm{m}$ [59, 395]. Some protein called tau (see section 8.4) has a bundling function and leads to a parallel arrangement of the axonal MTs in an imperfect hexagonal lattice (see Fig. 28) with spacing of $26.4 \pm 9.5 \mathrm{~nm}[72,312]$. An electron micrograph showing the arrangement of MTs under the influence of tau can be found in [72].

The MT network in the axon is complemented by actin networks, mainly located close to the cell surface. Actin filaments could also provide tracks for transport in regions with few MTs, as the axon terminal [243].

There are also neurofilaments present between the MTs, especially in axons of large diameter [72, 58].

\subsubsection{Dynamics of the axonal microtubule network}

In contrast to non-neuronal cells, the minus-ends of axonal MTs are not attached to the centrosome or one of the other common nucleating structures. Still, it seems that, in general, only the plus-end of axonal MTs is dynamic while the minus-end seems to be stable [28, 27, 227], probably due to some capping protein [87]. For example, $\gamma$-tubulin has been recently found to be present in the axon and might both play a role in the nucleation of axonal MTs and serve as cap on the minus-end [354] ${ }^{16}$. Recently were discovered some protein families that can associate with free MT minus-ends and stabilize them against total depolymerization [172].

\footnotetext{
${ }^{16}$ It should however be kept in mind that, though minus-ends of axonal MTs are usually supposed to be stable, treadmilling has also been observed in vivo [386], and it cannot be excluded that it also occurs in axons.
} 


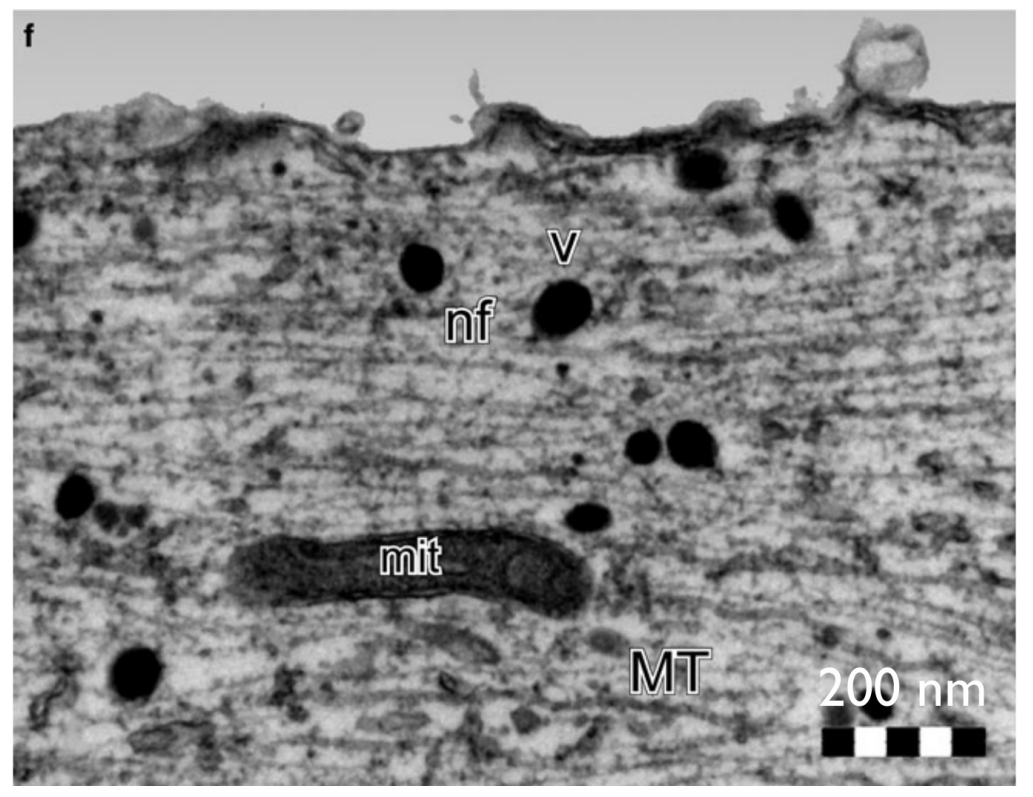

Figure 30: Highly magnified view of a small part of the axon obtained from electronic microscopy. Microtubules (MT), neurofilaments (nf), vesicles (v) and mitochondria (mit) are visible. Reprinted from [344].

Polymerization and depolymerization of the plus-end is about a factor two slower in mature axons than in non-neuronal cells and occurs along the main axis of the axon [353, 343].

It recently became possible to visualise the dynamics of MTs directly in living neurons using fluorescence and confocal microscopy techniques (see for example Movie S1 in [342]). [342, 343, 344] report some experiments in which some mRNA encoding for the plus-end-tracking protein EB3 tagged by GFP is injected in the cell body of cultured Aplysia neurons. EB3-GFP has the property that it binds transiently to dynamically polymerizing MT plus-ends. Images show comet-tail-like traces (see Fig. 29), which can be interpreted as the growing part of fixed MTs (though it can also be a signature for the motion of short MTs transported along longer MTs). The number of comet tails gives an estimate for a lower bound of the number of polymerizing MTs (not all polymerizing plus-ends are visualized).

These images renew the classical view of a static highly interconnected MT axonal network. Instead, plus-ends of MTs are polymerizing and depolymerizing very actively, a feature that allows to reconcile the antinomic requests for structure survival and adaptation capacity. However, while MT dynamics is quite well known in in vitro conditions, we are still lacking a full picture of MT dynamics in in vivo environments.

\subsubsection{Confinement}

Many results obtained in vitro cannot be directly applied to in vivo transport. One reason is that the interior of a cell is very crowded, and diffusion is strongly limited by steric hindrance [230]. An example is given in Fig. 30 which shows the internal structure of an axon. The size of common vesicles ( $\approx 100 \mathrm{~nm}$ [127]) is not negligible in front of MT interspacing. Neurofilaments also limit the available space [58].

The assumption made in section 6.2 of an infinite diffusion rate is not justified in this context. One may argue, however, that between two episodes of microtubule based transport, a given cargo may attach to the actin network: though each actin filement is polarized, there is no global alignment of the polarization as for MTs, so the cargo could undergo bidirectional motion by changing filament. Diffusion should then account for some motor-driven diffusive motion with a diffusion constant that depends on the speed of the cargo-motor complex and detachment rate on actin [348]. Though this mechanism may increase the longitudinal diffusion constant, first it does not make it infinite, and second it should be relevant only for cargos that can reach the actin network, located mostly along the membrane of the axon, and not for those travelling in the bulk part of the axon.

For cargos of intermediate size, confinement will limit strongly the diffusion around the MT (in the radial direction), 


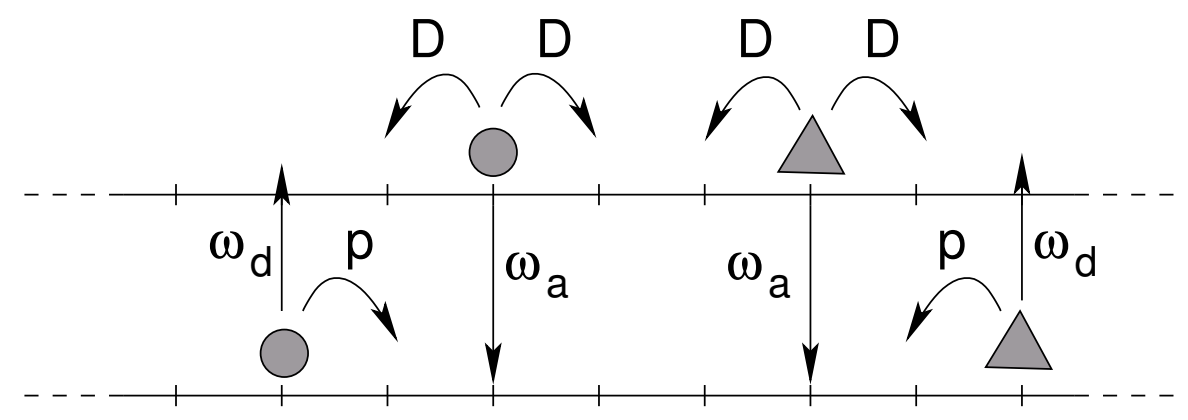

Figure 31: Two-species TASEP coupled to a diffusive lane. The model consists of two periodically closed one-dimensional lattices. The lower lattice serves as track for directed motion of the two oppositely moving particle species (represented respectively as disks and triangles) which interact via hard-core exclusion on this lane. The upper lane is a diffusive environment with no biased motion and without any interaction between particles. As a result, one site of the diffusive lane can be occupied by several particles at the same time. From [107].

and diffusion will occur mostly in the longitudinal direction, along the MT. This is the case that will be considered in the next subsections.

It must still be kept in mind that much larger cargos can be transported, e.g. mitochondria, which are too large to travel in the empty space between MTs and require a local rearrangement of MTs during their processive phase. We shall address this issue later in section 3.3.4.

Confinement may also have an effect on the dynamics of the MTs and on the overall structure of the axonal MT network. Indeed, a MT can grow only if there is empty space available for that. An example of such a steric effect on MT growth will be given in section 3.4.

\subsection{Finite diffusion: a two-species TASEP coupled to a diffusive lane}

In a living cell, the cytoplasm is filled with structures of different sizes such that diffusion is limited and actually dependent on the size of the diffusing object [231, 295]. Furthermore, vesicles in axonal transport usually originate at the synapse or in the cell body, so that conservation of transported particles is provided within the axon. Eventually, in real cells, more than one motor species is present. Especially in the axon, different motor families ensure anterograde and retrograde transport. Thus at least two types of motors have to be considered, moving in opposite directions on the MTs to capture the bi-directional nature of axonal transport.

In the next sections, we shall address this question of finding a minimal model that could lead to efficient bi-directional transport while retaining some basic constraints imposed by the axonal environment, namely confinement and particle number conservation.

A first attempt to include all these ingredients in a single model was performed in [109] and we shall now summarize the results.

\subsubsection{Model definition}

Instead of describing the full diffusion reservoir, it can be instructive to consider a simplified version of it, as proposed in $[109,152]$.

The model consists of two parallel one-dimensional lattices with periodic boundary conditions (figure 31). While one lattice represents the filament along which transport is carried out, the other mimics a diffusive environment in which no directed transport takes place. This corresponds to the cytoplasm which surrounds the microtubules and within which axonal cargos diffuse if not attached to one of the MTs. Particles interact via hard-core exclusion on the filament along which they hop in their preferential direction with rate $p$. On the diffusive lattice, particles hop in both directions with equal rate $D$ and do not interact with each other such that a single site on the diffusive lattice can be occupied by multiple particles at the same time. Particle exchanges between the two lattices occur at rate $\omega_{\mathrm{a}}$ and $\omega_{\mathrm{d}}$, corresponding to attachment and detachment moves to and from filament, respectively.

Whereas the motivation for the hopping rules on the filament has been discussed in previous sections, the particular choice of the diffusive lattice needs some clarification: As discussed in section 7.1.3, diffusion of axonal cargos in the 
cytoplasm is limited due to the size of the cargos and to the confinement imposed by the amount of structures present in the axon. After detachment from the filament, an organelle might therefore not be able to diffuse away from the filament in radial direction. The diffusive lattice can be seen as representing a narrow cylindrical region of cytoplasm surrounding the MT and within which unbound particles are confined. The hopping rate on the diffusive lattice is related to longitudinal diffusion, i.e. in the direction parallel to the MT filament. The limitation of diffusion in the transverse direction (i.e. turning around the MT) is not yet included into the model, so that the model allows multiple particles to occupy the same site on the diffusive lattice. Therefore, organelles do not feel the local density of axonal cargo and can pass in front of each other if they are not attached to the filament. The model consequently overestimates this bypassing in the narrow environment and the results are likely to underestimate the jamming in a real system with strong spatial confinement.

The generic result of this model is that a huge cluster is formed which absorbs almost all the particles of the system. This effect is due to the highly inefficient bypassing of obstacles on the filament. A particle which encounters an obstacle such as a cluster of particles on the filament would have to detach to the diffusive lane and then diffuse over the obstacle in order to re-attach to the filament on the other side. Being unbiased, the diffusive motion is quite inefficient, especially when the obstacle becomes longer. As a result, a cluster on the filament is extremely stable for large system sizes.

This cluster formation does not depend on the density but on the number of particles as only a limited number of particles is necessary to form a nucleating cluster which will then start to grow indefinitely. It therefore has been reasoned in [109] that the model exhibits macroscopic clustering at any finite density $\rho_{\text {tot }}^{+}=\rho_{\text {tot }}^{-}>0$ in the limit of infinite system sizes $L \rightarrow \infty$ (See [105] for details).

High values of the diffusion rate $D$ decrease the tendency to form macroscopic clusters and actually totally suppress it in the limit $D \rightarrow \infty$. Indeed, this limit case correspond to the models of section 6.2 , for which diffusing particles loose memory of their last attachment site. This underlines that systems where particles have some sort of memory of their last site of attachment to the filament behave quite differently and require a specific study.

The effect of multiple transport filaments in parallel has also been considered by enlarging the model to have several subsystems as in figure 31 put next to each other. Particles may change from one subsystem to the other either via the diffusive lanes or by moves from filament to filament. Counter-intuitively, this coupling of multiple filaments has turned out to promote the formation of clusters although additional ways for the bypassing of obstacles are available. The moves from subsystem to subsystem transport locally high densities from one filament to the other, leading to a system which is blocked on all filaments at approximately the same horizontal position.

Taken together, these results indicate that a simple bypassing mechanism via a diffusive lane does not help in making bidirectional transport efficient and overcoming the blocking of particles of opposite charge on the filament. In real systems, the consequences should be even worse, since these systems are open at their ends so that the number of particles in the system is not limited as for the periodically closed system considered here.

\subsubsection{Varying confinement}

The jamming described in the previous section 7.2 strongly relies on the fact that radial diffusion is inhibited. Actually the degree of confinement in the axon is not precisely known, and may depend on the type (and size) of the cargos under consideration.

It is possible to increase effectively the radial extension of the diffusive reservoir just by lowering the attachment rate. If we assume that the diffusive environment has the shape of a cylinder of radius $R$ centered around the MT, a simple scaling argument indicates that the effective attachment rate should vary as $\frac{\omega_{a}}{R^{2}}$. When the diffusive reservoir is made larger, the total number of particles must also be increased so as to keep a constant density.

In [106], the effect of a larger cytoplasmic environment was considered in this way. We could show a crossover from a jamming regime for strong confinement, to a free flow regime in the case of a large diffusive reservoir. However, in the latter case, as particles spend most of their time unbound, the overall net transport is not very efficient. Besides, we expect confinement to be quite severe in the axon. Thus it is still crucial to understand how bi-directional transport in a confined environment can be regulated to avoid desastrous jamming.

A naturally arising question therefore is: What are the minimal prerequisites in order to maintain bidirectional stochastic transport of interacting particles in small volumes if site exchange on the track is not possible?

Several mechanisms can be considered to provide efficient bi-directional transport. In the following sections, we shall explore two possible families of scenarios. One of them would be that interparticle interactions lead to lane formation. We shall consider this scenario in section 7.4. However, we shall see that it is not obvious that this mechanism alone could achieve efficient transport in the context of axonal transport. Another scenario, perhaps a priori less intuitive, based on the 


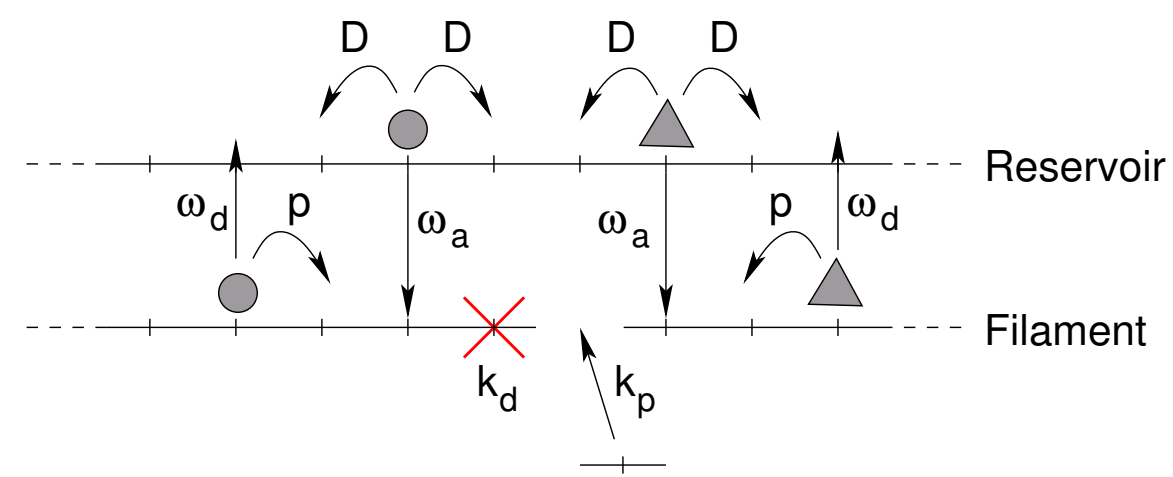

Figure 32: Model for bidirectional transport with simplified uncorrelated filament dynamics. Same legend as Fig. 31, with the dynamics of the lower filament added. A filament site is eliminated at rate $k_{\mathrm{d}}$ and recreated at rate $k_{\mathrm{p}}$. From [108].

dynamics of the underlying lattice, will be presented now. We will show that it could also be a key ingredient for efficient transport. The advantages and drawbacks of each scenario will be discussed.

\subsection{Lattice dynamics}

As presented in the previous section, generic problems occur when considering bidirectional transport on a static lattice surrounded by a confining diffusive reservoir, if particles interact via exclusion. On the other hand, real axonal transport is very efficient although it is bidirectional and molecular motors exclude each other from MT binding sites.

In the present section, we shall present a mechanism that can lead to efficient bidirectional transport on a single track through consideration of the filament dynamics [107, 106, 108]. This extension of the model of section 7.2 has been inspired by the experimentally observed dynamics of the cytoskeleton (see section 7.1.2). In fact, the MTs on which molecular motors move are themselves highly dynamic, due to nucleation, polymerization and depolymerization, which occur on time scales similar to those involved in motor transport and are thus likely to interfere with the motor dynamics.

Beyond the interest for intracellular traffic, the present section gives an example where a dynamically driven jammed phase is hindered by the lattice dynamics.

\subsubsection{Idealized MT dynamics: model definition}

The model is a modified version of the two-lane model presented in section 7.2 (figure 31). As a new feature we add some lattice dynamics for the lower lane, i.e., some sites are eliminated and recreated. The diffusive upper lane remains unchanged. On the lower lane, particles can only occupy a site if this binding site exists. The attachment moves (rate $\omega_{\mathrm{a}}$ ) are consequently rejected if the binding site has been eliminated. Additionally, a particle will automatically switch to the upper lane if it makes a forward step (rate $p$ ) onto an eliminated site or if the site which is currently occupied by the particle is eliminated. The whole system is closed with periodic boundary conditions. In the following, the state of the filament lattice is given by the local lattice states $\tau_{i}$ which take the value zero if site $i$ is eliminated and one if the site exists.

Different types of lattice dynamics can be considered. We shall as a starting point take a very simple one, and discuss the robustness of our results against other choices for the lattice dynamics later in sections 7.3.4 and 7.3.5.

Uncorrelated lattice dynamics. This dynamics consists of eliminating randomly a site of the filament with rate $k_{\mathrm{d}}$ and recreating eliminated sites with rate $k_{\mathrm{p}}$ (figure 32) where the indices are reminiscent of the processes of MT polymerization and depolymerization. Here, the evolution of the lattice state $\tau_{i}$ is not influenced by the particle dynamics and can be easily expressed as:

$$
\frac{\partial\left\langle\tau_{i}\right\rangle}{\partial t}=k_{\mathrm{p}}\left(1-\left\langle\tau_{i}\right\rangle\right)-k_{\mathrm{d}}\left\langle\tau_{i}\right\rangle
$$

In the stationary state, one therefore has a homogenous density of filament sites given by $\left\langle\tau_{i}\right\rangle=k_{\mathrm{p}} /\left(k_{\mathrm{p}}+k_{\mathrm{d}}\right)$. 

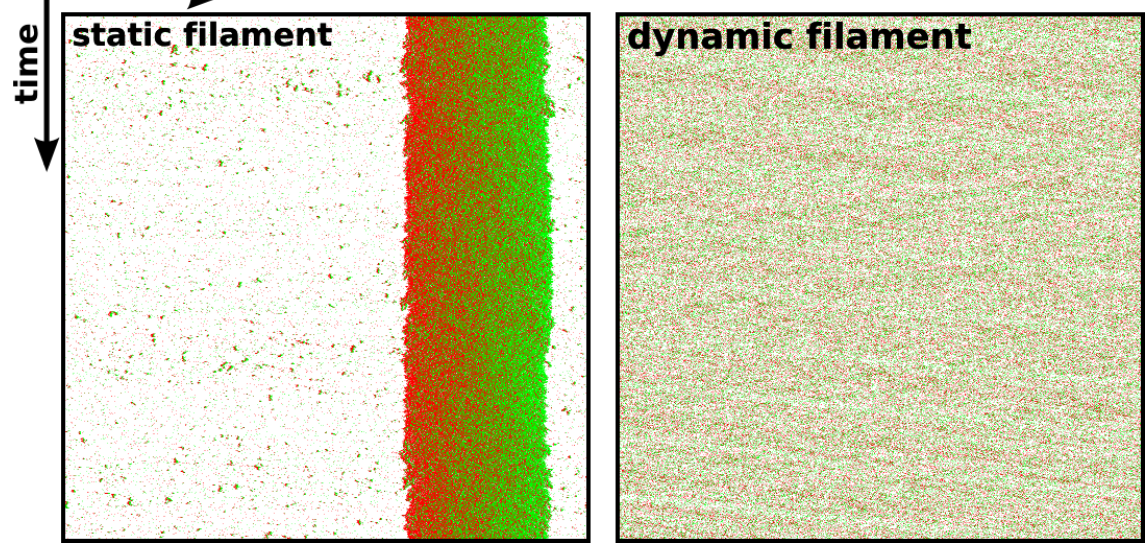

Figure 33: Space-time plots on a static and a dynamic filament. Every line in the images corresponds to the configuration of the filament lattice in a system of length $L=1000$ with positive particles in red and negative particles in green. Vacant lattice sites are white. The static lattice plot has been recorded at $k_{\mathrm{d}}=0$ and the dynamic lattice plot at $k_{\mathrm{d}}=0.2$, with uncorrelated lattice dynamics. From [107].

Though this dynamics does not pretend to be realistic, the link with axonal transport can be thought by considering that the lower filament does not represent only one MT, but several MTs following each other end-to-tail. The elimination of one lattice site would then be equivalent to the depolymerization of a plus-end MT tip. This lattice dynamics could also model localized obstacles on the microtubules that oblige the motors to detach [361].

It is obvious that a much more complex model should be developped to model realistically axonal transport. Here, we rather take this simplistic uncorrelated lattice dynamics as a minimal tool to demonstrate some possible generic effects of lattice dynamics.

\subsubsection{Phase transition to a homogenous state}

We shall first explore numerically the properties of the model with uncorrelated lattice dynamics.

The results presented on this model therefore come from numerical Monte Carlo simulations obtained over at least $10^{6}$ Monte Carlo sweeps for $p=1, D=0.33, \omega_{\mathrm{d}}=0.02$ and $\omega_{\mathrm{a}}=0.33$. Again, $p \gg \omega_{\mathrm{d}}$ is chosen in order to capture the processivity of molecular motors. The polymerization is fixed at $k_{\mathrm{p}}=1$ if not stated otherwise and $k_{\mathrm{d}}$ is usually varied in order to observe the effects of the lattice dynamics with $k_{\mathrm{d}}=0$ corresponding to a static lattice. The density of particles is fixed at $\rho_{\text {tot }}=2 \rho_{\text {tot }}^{+}=2 \rho_{\text {tot }}^{-}=1$ in order to have clustering on static lattices in numerically accessible system lengths.

The effect of lattice dynamics is very visible on the spatio-temporal plots of Fig. 33. For a static lattice $\left(k_{\mathrm{d}}=0\right)$, a macroscopic cluster is formed which seems quite stable in time. No such cluster is observed in the simulation with lattice dynamics (with $k_{\mathrm{d}}=0.2$ ). Instead, the spatio-temporal plot is rather uniform. Indeed, the cluster distribution of Fig. $34 \mathrm{~A}$ confirms that for $k_{\mathrm{d}}$ sufficiently large, large clusters disappear.

The central observation is seen on Fig. $34 \mathrm{~B}$, showing the particle current as a function of $k_{\mathrm{d}}$, which can be seen as the dynamics strength. For $k_{\mathrm{d}}=0$, i.e. for a static lattice, the particle current is quite low and decreases when the lattice size increases. Actually, as explained in section 7.2, the flow depends on the total number of particles in the system. This damaging feature persists for low $k_{\mathrm{d}}$ values, though for a given system size the current increases with $k_{\mathrm{d}}$. The interesting point is that beyond a certain $k_{\mathrm{d}}$ threshhold, the current becomes system size independent. Actually it depends then rather on density.

The entire curve is not shown; of course, for very large $k_{\mathrm{d}}$ values, the filament is so much destroyed that the current must decrease again. So there is an optimal value of $k_{\mathrm{d}}$ somewhere in the middle, which does not depend strongly on the value for $k_{\mathrm{p}}$. The disappearance of the large cluster is accompanied by a transition from a size-dependent to a sizeindependent state (figure $34 \mathrm{~B}$ ). 
A

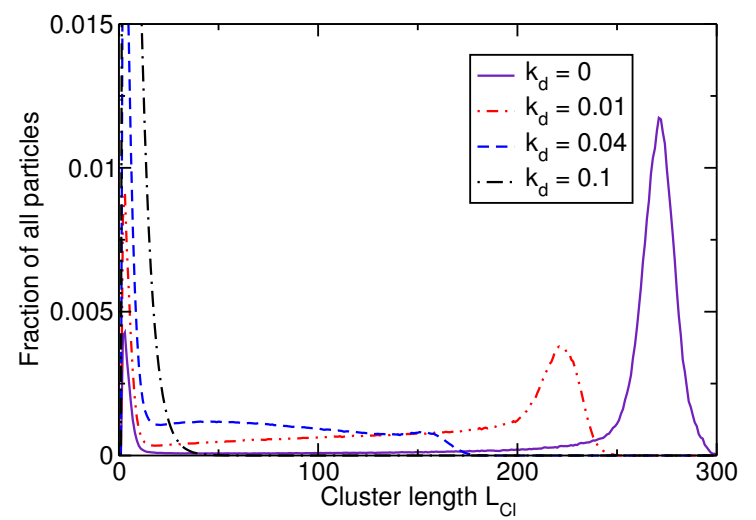

$\mathrm{B}$

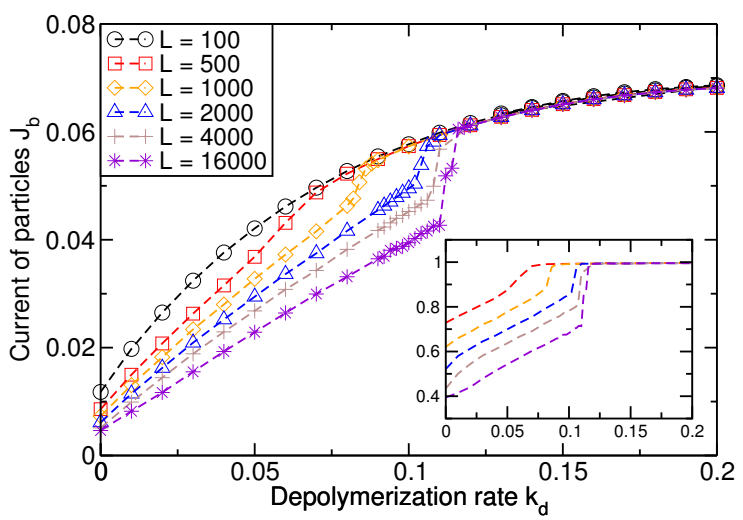

Figure 34: Cluster distribution and $(B)$ current in the basic model with additional lattice dynamics. $(A)$ The cluster distribution shows the fraction of all particles in the system which are found in a cluster of a given size $L_{\mathrm{Cl}}$. (B) The current of positive particles bound to the filament $J_{\mathrm{b}}$ is shown for different system sizes $L$ as a function of the depolymerization rate $k_{\mathrm{d}}$. Since both particle species behave symmetrically, the current of negative particles takes an identical form. The inset shows the same data divided by the current in the smallest system $L=100$. From [107].

\subsubsection{Mean-field approximation}

For the stationary state, in the frame of a mean-field approximation assuming factorization of correlation functions and translational invariance, one obtains the relation between bound and unbound densities for each motor type

$$
\omega_{\mathrm{a}} \frac{k_{\mathrm{p}}}{k_{\mathrm{p}}+k_{\mathrm{d}}} \rho_{\mathrm{u}}^{ \pm}\left(1-\rho_{\mathrm{b}}^{+}-\rho_{\mathrm{b}}^{-}\right)=\left(\omega_{\mathrm{d}}+p \frac{k_{\mathrm{d}}}{k_{\mathrm{p}}+k_{\mathrm{d}}}+k_{\mathrm{d}}\right) \rho_{\mathrm{b}}^{ \pm} .
$$

The 1.h.s. (respectively r.h.s.) gives the flux of particles attaching to the filament (resp. away from the filament). One can thus define effective detachment and attachment rates

$$
\omega_{\mathrm{a}, \text { eff }}:=\omega_{\mathrm{a}} \frac{k_{\mathrm{p}}}{k_{\mathrm{p}}+k_{\mathrm{d}}} ; \quad \omega_{\mathrm{d}, \mathrm{eff}}:=\omega_{\mathrm{d}}+p \frac{k_{\mathrm{d}}}{k_{\mathrm{p}}+k_{\mathrm{d}}}+k_{\mathrm{d}},
$$

where the case of the static lattice is recovered as a limit case. The effective attachment rate is thus lowered by the lattice dynamics while the effective detachment rate is increased. The global effect is that the particles have a lower effective affinity to the filament.

It should be noted however that for a static lattice, considering only a lower affinity to the filament has been shown to reduce but not eliminate the tendency of cluster formation [109]. The mean-field approach cannot capture the main effect of lattice dynamics, which prevents the growth of clusters. Indeed, for the value of $k_{\mathrm{d}}$ that optimizes the flow, blocking situations with at least two particles of different species still occur frequently. As a consequence, the maximum currents that are obtained are about one third of the flux in a comparable single-species system [196], which is nevertheless a great improvement compared to the static filament case.

Further understanding of this system could be obtained from an analysis similar to the one in [270] for the symmetric motion of non-interacting particles in fluctuating energy landscapes. Here the effective 'potential' landscape emerges spontaneously from the particle jamming.

\subsubsection{Rubustness against different lattice dynamics}

It is an open question whether the main result of section 7.3.2, i.e., the phase transition to a density-dependent state with efficient transport in a finite system is independent of the exact choice of lattice dynamics. In this section, in order to test the robustness of our results, we consider different simplistic lattice dynamics which incorporate different generic ingredients. 
Lattice dynamics correlated with particle occupation. It was shown that some motors do have an influence on the dynamics of MTs and contribute to the regulation of the MT length [164], though the mechanisms of such a regulation are not fully understood yet. Though it is not clear whether this effect is relevant for axons, and though the MT-depolymerizing kinesins are usually not responsible for intracellular long-range transport, this observation inspired another simplified lattice dynamics correlated now with particle occupation.

The lattice dynamics rules are the same as for the uncorrelated lattice dynamics, except that now, a site is eliminated with rate $k_{\mathrm{d}}$ only if that site is occupied by a particle.

The behavior of the system with this correlated lattice dynamics is qualitatively the same as for uncorrelated lattice dynamics. The main difference is that the flux in the homogenous phase is higher than in the first scenario. Indeed, when a motor is moving freely, it encounters less holes than in the case of the uncorrelated lattice dynamics, as empty lattice sites cannot be eliminated.

A variant in which several successive lattice sites must be occupied to allow depolymerization exhibits also the transition to a density-dependent efficient state.

Treadmilling lattice dynamics. We have also considered some idealization of treadmilling (see section 2.1), though this type of motion is actually of minor importance in axons, but not necessarily in other biological systems: In the initial configuration, some lattice sites are suppressed so as to create equally spaced holes on the filament. During the time evolution, these holes propagate synchronously but stochastically through the system. The aforementioned transition toward efficient transport is still observed. It is to be noted that, since the holes move only in one direction, the two species of particles are affected differently. For both species, there is a considerable increase of the flux and a maximum current comparable as for previous lattice dynamics is reached.

\subsubsection{Toward more realistic MT network and dynamics}

The models presented so far are very simplistic in the description of the microtubule network. Some more realistic description of the geometrical organization of the microtubules would be useful to check whether the results obtained on isolated filaments still hold.

We have seen that in the axon, transport occurs on many parallel microtubules. In some preliminary work, we have implemented the model of Fig. 31 for bidirectional transport on a network of parallel MTs placed on a hexagonal lattice similar to the axonal MT structure of Fig. 28. Besides, the simplified uncorrelated lattice dynamics of Fig. 32 is applied only on a finite fraction of each MT length. Still, the transition towards efficient transport described in section 7.3.2 is still observed. The next step, under development, is to consider more realistic MT dynamics, with explicit description of the dynamic instability and of MT transport.

\subsection{Motor-motor interactions}

Some other scenarios could be considered to account for the efficiency of bidirectional intracellular trafic. A quite natural one would be to include motor-motor interactions. In particular, one could expect that if these interactions can lead to lane formation, bidirectional transport could be quite efficient.

We shall first review some experimental facts about motor-motor interactions, and then some modeling efforts along these lines.

\subsubsection{Experimental observations}

There are much more results on mutual interactions for kinesins than for dyneins, as the latter are more difficult to handle experimentally. Still, it is established that dynein can quite easily side step to a neighbouring proto-filament [383, $384,313]$, while there is no clear agreement on side-stepping for kinesin.

In some experiments, it has been shown that kinesins stay tightly bound to the MT and wait when being blocked by an obstacle [337]. Although the general direction of kinesin motion is along individual protofilaments [301, 41], recent experiments seem to show that occasional side steps onto neighboring protofilaments of the same MT are possible. Telley et al [361] have decorated some MTs with obstacles that occupy exactly one binding site of a kinesin. They observe that the average run length of kinesins along the MT is much longer than the average interdistance between obstacles. Thus kinesins have a low but finite probability to pass obstacles. The authors suggest as the most probable interpretation of this fact that kinesin can change protofilament, though another explanation could be that kinesins detach and reattach shortly after, while being kept weekly bound with the obstacle. Some experiments reported by Dreblow et al [100] confirm 


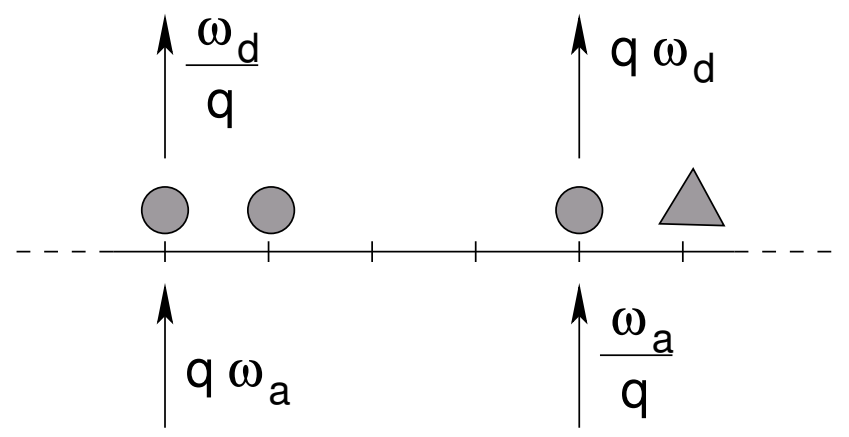

Figure 35: Particle-particle interactions in a two-species TASEP, as studied in [197]. The attachment (detachment) rate increases (decreases) by a factor $q$ if the next site is occupied by a particle of the same species. In the case of a particle of the opposite species, detachment is enhanced and attachment is lowered.

the ability of kinesin to bypass obstacles (fixed or slowly moving). The width of side steps seems compatible with the single kinesin heads steps [100, 394]. Side-stepping still remains a rare event for kinesin-1, while some representatives of the kinesin-2 class were found in vivo to perform left-handed spiraling around microtubules [61]. Anyhow, the general conclusion is that kinesin side-stepping, if it occurs at all, is far less frequent than for dynein.

Another type of interaction has been evidenced between kinesins. Indeed, for conventional kinesin, there is evidence for a mutual attractive interaction between motor proteins, so that a kinesin preferentially binds to the MT in the vicinity of other kinesins $[375,268,311]$. The strength of the interaction between two kinesin-1 molecules has been estimated at $1.6 \pm 0.5 k_{B} T[311]$.

\subsubsection{Modelling modified attachment rate}

Based on the aforementioned preferential binding of kinesin near other kinesins, Klumpp and Lipowsky [197] have proposed a model with modified attachment and detachment rates along the filament (figure 35). While the motivation for taking this modified rates comes from the decoration experiments with kinesin mentioned in section 7.4.1, this concept is generalized here to apply for both particle species which are furthermore supposed to repulse each other reciprocally.

Klumpp and Lipowsky take as a starting point a two-species version of the model described in Fig. 22 of section 6.2 (considered here with periodic boundary conditions). Without specific attachment/detachment rates, this two-species version would lead to jamming on the filament.

When the modified rates of Fig. 35 are used, spontaneous symmetry breaking occurs for $q>q_{\text {crit }}$, for which one particle species is completely excluded from the filament while the other undergoes one-species directed motion. If one considers the possibility of multiple filaments in the system, some filaments are be populated with one motor species, and the others by the other motor species.

As we said, this interesting lane formation effect was found [197] for a reservoir with infinite diffusion rates, and with unbound number of particles (grand-canonical reservoir). However, we found later that for this type of reservoir, lane formation occurs at such high densities that transport is not efficient, because of intra-species hindrance on the filaments [108].

Actually, the behavior of such models depends strongly on the type of diffusion reservoir that is considered, as we already showed in previous sections. Thus we explored the behavior of the model also for a canonical reservoir (infinite diffusion but fixed total number of particles) and for single-lane diffusion reservoir as in Fig. 31 (finite diffusion and particle conservation).

Table. 1 summarizes the results that we found [108]. We confirm that, for modified attachment/detachment rates, lane formation is obtained both for grand canonical and canonical reservoir. Lane formation implies efficient transport only in the case of a canonical reservoir, and if the number of motors is low enough so as to avoid the filaments to become crowded.

Lane formation can be obtained only if the interaction strength $q$ is large enough. There is no direct measurement of this interaction strength yet. Very crude estimates made from the experiments in [268] and [311] would give too low values. However, more experimental data would be necessary before giving a conclusive statement. 


\begin{tabular}{|c|c|c|}
\hline & Static lattice & Dynamic lattice \\
\hline $\begin{array}{c}\text { Infinite diffusion rate } \\
\text { Grand-canonical reservoir }\end{array}$ & Lane formation [197]. & $\begin{array}{l}\text { No lane formation } \\
\text { for strong dynamics. } \\
\text { Current increase only } \\
\text { due to lattice dynamics. }\end{array}$ \\
\hline Infinite diffusion rate & Lane formation. & $\begin{array}{l}\text { No lane formation } \\
\text { for strong dynamics. }\end{array}$ \\
\hline Canonical reservoir & $\begin{array}{l}\text { Very efficient } \\
\text { at low densities }\end{array}$ & $\begin{array}{l}\text { Then current increase only } \\
\text { due to lattice dynamics. }\end{array}$ \\
\hline Finite diffusion & $\begin{array}{l}\text { No lane formation. } \\
\text { Very weak current increase } \\
\text { due to the interaction. }\end{array}$ & $\begin{array}{l}\text { No lane formation. } \\
\text { Strong current increase } \\
\text { due to lattice dynamics. }\end{array}$ \\
\hline
\end{tabular}

Table 1: Summary of the model behavior with the modified attachment and detachment rates of Fig. 35. The central column corresponds to static filaments, while for the right column, both modified attachment/detachment rates and uncorrelated lattice dynamics were implemented.

(a)

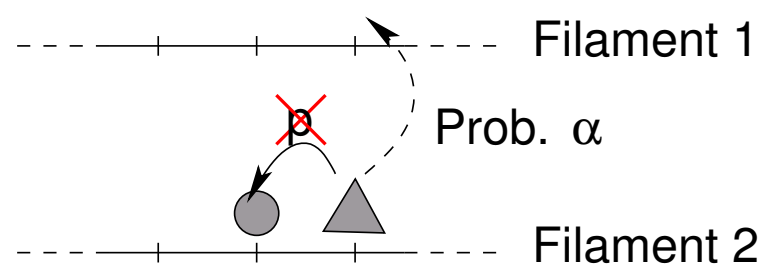

(b)

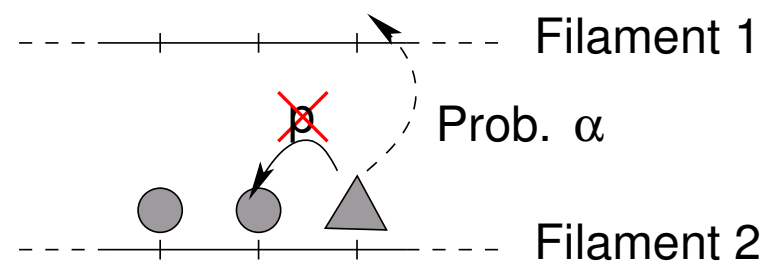

Figure 36: Modelling side stepping in front of an obstacle. A particle changes lane with rate $\alpha$ if it is blocked by (a) one or (b) two particles of the other species. The reservoirs are not shown. From [108].

The most important drawback of this scenario based on lane formation is that it does not seem to survive under confinement [108]. However, infinite diffusivity seems an unrealistic assumption for intracellular traffic. Thus there is a interest in finding alternative scenarios that would work in confined environments. We explore in the next section some other kind of motor-motor interactions.

\subsubsection{Modelling side-stepping}

As seen in section 7.4.1, dyneins may side-step to a neighboring proto-filament and it is likely that kinesin can also do so when meeting an obstacle, though it remains a rare event.

We consider a variant of the model of Fig. 31 with finite diffusion, with several parallel two-lane subsystems, and with the possibility for motors to change filament when they meet motors of another species (see Fig. 36 for details of the rules) [108].

When the rule of Fig. 36(a) is used, lane exchanges are too numerous, and no great current improvement is obtained. By contrast, the more constrained lane changing rule of Fig. 36(b) does lead to lane formation and efficient transport.

Interestingly, when uncorrelated lattice dynamics is added to these side-stepping rules, it promotes lane formation such that lane formation can be obtained even for the rule of Fig. 36(a). Besides, as we already saw in section 7.3.2, lattice dynamics by itself can improve current, with a higher efficiency than obtained by one-obstacle side-stepping.

\subsection{Discussion}

The examination of the models in this chapter yields the general result that it is not trivial to observe bidirectional transport of particles which move along the same track, if motor exclusion and finite diffusion are taken into account.

Though the nature of motor-motor interactions still has to be explored experimentally, we have shown that it is possible to find some interactions that make bidirectional transport efficient even under confinement - an appealing scenario as it proposes a very easy solution to achieve long-range transport by local interactions. It should be possible to design 
experiments with molecular motors and microtubules to demonstrate lane formation - if the interactions between molecular motors turn out to be strong enough.

We also reported in section 7.3 that a transition towards efficient transport is obtained when the underlying lattice itself is dynamical. This rather counter-intuitive result - transport is more efficient when parts of the roads are suppressed - still needs to be confirmed under more realistic modeling efforts of axonal transport.

However, several experimental observations seem to confirm the strong link between MT dynamics and axonal transport. In medicine, a possible neuropathy following the treatment of cancer by MT-stabilizing drugs such as paclitaxel or docetaxel is a well-known phenomenon [188] and presumably linked to the breakdown of axonal transport [122]. Recently, in vivo experimental imaging has shown that the breakdown of MT dynamics induced by the injection of paclitaxel indeed coincides with the breakdown of vesicle transport [343]. Though this does not prove that one is the cause of the other, these experiments are strong indications of an interplay between MT dynamics and transport.

An important remark is that scenarios for efficient transport based on motor-motor interactions or on lattice dynamics should not be thought as competing scenarios. On the contrary, we found in many cases that both contribute in a cooperative way to the global efficiency of transport.

In this chapter, we have discussed that efficient transport is based on the control of jammed configurations which naturally arise in bidirectional transport under strong confinement. A full modeling of motor driven transport in axons is not accessible yet since much information is still lacking, concerning for example motor interactions or the details of axonal MT dynamics. A few of the open questions will be listed in section 8 . However, many informations should become available in the coming years and allow for more accurate modeling.

\section{Challenges for the development of transport oriented models}

In the present chapter we try to address some important open problems that may have strong impact on the transport of cargos in cells. Though these problems are general for all cells, they are particularly relevant for axons, which are so long and so crowded that active transport is essential. Therefore, our discussion will be oriented towards axonal transport but the reader should keep in mind that most problems that are considered here are also encountered in other cell types.

\subsection{Cargo transport in axons and protein synthesis}

The axon provides a crowded environment where a large variety of cargos has to be transported. A cargo is often not transported by a single but by teams of molecular motors. This is in particular true for large objects.

We have seen in section 3.3 that even the transport of a single cargo by teams of motors is not fully understood yet. Once the characteristics of bidirectional cargo transport will be established the next task will be to consider the collective effects of such cargo-motors complexes.

These have been partially considered in chapter 7, in which a particle can be seen as a coarse grained description for a whole motor-cargo complex. However, the bidirectional nature of the cargos' trajectories and its role in collective motion has to be explored further. In particular, the tendency to have jamming can depend on the variability of trajectory bias between different cargo types.

The dynamics of cargos can also be considered from a functional point of view. The cell needs a certain number of proteins to be synthesized, transported at the right place where they will be used, and then recycled or degradated. Miller and Heidemann [253] list a number of open questions related to transport and synthesis of proteins. In particular, it is not clear yet how very long axons are supplied with proteins. It is also not known where protein degradation occurs along the axon, if it is relevant at all.

The original picture was that all proteins were synthesized in the soma, and then transported where needed. But several experiments have indicated that mRNA replication also occurs locally along the axon [346, 289, 347]. There are still many open questions about the spatial localization of axonal protein synthesis - e.g., does it occur in specific axonal compartments? - and about the triggering of mRNA translation [289]. The tip of the axon in particular should be a place where the translation of mRNA occurs, to comply with the needs of axonal growth. Protein synthesis at the distal end cannot be completely autonomous from the cell body, and some retrograde signaling transport is needed, for example to inform the cell body that the axon has been injured [377]. Thus protein synthesis and transport are necessarily highly linked, and much more understanding of these coupled phenomena is needed. 


\subsection{The transport network: microtubule dynamics in axons}

The recent discovery that a large number of MT plus-ends are dynamic in axons, even in mature neurons, considerably changed the picture of the axonal MT network, as we already pointed out in the previous chapter. This finding immediately raises questions about the global dynamics of the axonal MT network.

In axons this question is not easy to answer, since the minus-ends are not bound to a MTOC as in other mammalian cells. Therefore, it would be very interesting to visualize the spatio-temporal evolution of the MT network in axons. However, in contrast with cells where the MT network is very sparse and where a simple marking of tubulin allows to track the network structure and dynamics, this is not possible in axons due to the high density of MTs. A possible solution to this problem would be labeling of the plus- and minus-ends rather than the entire MTs. Until now, most of the in vivo experiments concentrate on the growing ends of MTs, which can be traced by a number of standard labels. By contrast, the information about the spatial and temporal distribution of microtubule minus-ends in axons is very sparse. Indeed, it is still an experimental challenge to find how to track the minus-ends or the shrinking plus-ends of MTs. The recent finding of families of proteins that specifically bind to the MT minus-ends opens new perspectives for the marking of minus-ends [172]. In the future, the simultaneous tracing of plus- and minus-ends would allow to get much more insight to the actual distribution of static and dynamic microtubules in axons.

Another issue is related to the role of steric interactions in MT network dynamics. It is known that obstacles can bend MTs, or limit their growth by inducing catastrophes. As the axon is very crowded a better knowledge of its inner structure would be necessary to take these steric interactions into account in a model of MT networks dynamics in axons.

The polymerization of MTs relies obviously on the supply of tubulin subunits. As growing MT plus-ends have been observed in every part of the axon, there is a need to provide new material to sustain the MT-structure over the whole lifespan of the axon. There is, however, an ongoing debate about how and under which form tubulin is transported. In order to illustrate possible mechanisms of tubulin supply we discuss some of the mechanisms that are presently discussed.

As already mentioned in section 8.1, the historical view is that proteins can only be synthesized in the cell body, and then transported to a location where they are needed. The length of axons prevents transport to be performed only by diffusive means, and active transport has to be organized [259]. It is possible that short assembled MTs are transported to the plus-end of growing MTs in the axon. Alternatively only tubulin subunits could be transported and then assembled at their arrival point. It is unclear whether there is a nucleating structure allowing for free tubulin to assemble into MTs directly in the axon. However, it is known from in vitro experiments that tubulin can spontaneously assemble even in the absence of a nucleating site if the tubulin concentration is high enough [167]. More recently it was found that minusends can be stabilized even if not connected to any MT nucleating site, through decoration by a specific protein family (CAMSAP) [172].

Some experiments seem to indicate that MTs can be transported as a whole $[26,3,5]$. Indeed, one knowns from other biological systems that molecular motors are able to displace MTs (e.g. in the mitotic spindle). The mechanism is the following: motors produce some relative motion between the cargo they carry and the MT they are stepping on. If the MT is strongly anchored, the cargo will move. However, if the cargo is fixed, for example if the cargo domain of the motor is attached to the actin cytomatrix of the axon, then it is the MT that could move [26]. Under such a scenario, cytoplasmic dynein could move the MTs either anterogradely or retrogradely depending whether its cargo domain is attached to the actin cytomatrix or to other MTs, though anterograde motion should dominate. Indeed, experiments have confirmed the crucial role of cytoplasmic dynein in MT movement [4].

The so-called cut-and-run model has been conceived by Baas et al. [30] to explain the maintenance of the stationary state of the axonal MT network: MTs nucleate and polymerize at the centrosome in the soma and are then cut by a MT-breaking protein such as katanin. Katanin has indeed been shown to be located in higher concentrations near the centrosome [5]. Short MT pieces are then transported through the axon with their plus-end pointing in distal direction [3, 25]. The motility is achieved through molecular motors, which are anchored on actin filaments or longer MTs and exert forces on the transported MT by walking on it. It has in fact been shown that dynein and the actin cortex are responsible for half of the anterograde MT transport in axons while the rest depends on kinesin [269, 4]. One thus finds two populations of MTs in the axon: short and mobile MTs which are transported and perform bidirectional motion and long and immobile MTs which serve as structures along which the short MTs can be transported. The long MTs are imagined to be immobile because of a large number of crosslinks to other axonal structures such as the neighboring MTs via tau [4, 31], though actually they are still active at their plus-end and might depolymerize at any moment. Experiments are contradictory whether the short MTs undergo dynamic instability during transport [25, 381] or whether they are stable [234, 4]. 
Furthermore, it is an open question if and how tubulin is transported retrogradely. Such motion has not been observed yet, but might be needed to balance the constant influx of tubulin in form of anterogradely transported MTs. Alternatively, tubulin might degrade at the synapse or be released to the extracellular space from there [281].

The conclusion of this section is that we are still lacking some global image of the living cycle of MTs in the axon, although it is confirmed that the plus-ends of axonal MTs are dynamic [343]. A more conclusive picture of the axonal MTnetwork would greatly improve the understanding of motor-driven transport in axons and support the further development of transport models.

\subsection{Motors action on MTs}

We have seen in chapter 7 how the MT dynamics could play a role in motor transport. Reciprocally, motors can influence MT dynamics.

Some other kinesins than kinesin-1 have been shown to be less implicated in intracellular transport but to have an influence on the MT dynamics. For example, members of the kinesin- 8 and kinesin-13 family are able to find the plus-end of MTs (and are therefore considered as +TIPs [8]) where they destabilize MTs and promote depolymerization by using the energy from ATP hydrolysis to deform the MT lattice until it breaks [94, 258, 360].

Exclusion processes have been used to model the collective behavior of such depolymerases [303]. The model predicts some collective effects through the jamming of polymerases, that would affect the depolymerization speed.

Other exclusion process based models have demonstrated how the activity of plus-end tracking molecular motors acting as depolymerases can regulate the length of filaments, which becomes then less sensitive to variations of the surrounding medium. These length regulation mechanisms were found both for treadmilling filaments [173], or for filaments attached to an MTOC [252].

Molecular motors can also have a stabilizing effect. For example, cortical dynein can bind to MT plus-ends and stabilize them, with a length regulation effect, as was confirmed by several in vitro experiments [157, 218, 217].

If MT network dynamics plays a crucial role in transport as proposed in chapter 7, the features presented in this subsection provide a feedback loop on MT dynamics. The transport of tubulin by motors - needed for MT polymerisation - is another one.

\subsection{Modification of MTs by MAPs : The example of tau proteins}

The MTs that we have considered until now were ideal ones, without any defect or any variation in their surface structure. This is not the case in vivo, where enzymes can modify tubulins units [168], or where MAPs can associate to the MT. The cell could possibly use inhomogeneities or MT decoration factors to control the motor transport properties. All these aspects should be integrated for a full description of the interplay between transport and tracks. In this section we shall discuss more thoroughly a specific type of protein that binds on the MTs, namely tau proteins. A similar role is played in dendrites by MAP2 proteins [36, 39].

The structure of both tau and MAP2 proteins has a MT binding domain and a hydrophobic part which extends into the surrounding cytosol [226]. The MT binding domain is able to bind at the outside of the MT cylinder as well as across protofilaments [9, 320]. The interactions between the hydrophobic parts of MAPs induce constraints on the structure of MTs (see Fig. 28). It is still debated whether these MAPs are "crosslinkers or spacers of microtubules" [248]. On the one hand, the hydrophobic parts of bound tau molecules are able to dimerize and to induce bundling of MTs [312]. Cross-links have been observed both in vitro [1] and in vivo [159, 226]. On the other hand steric constraints between MT-MAPs are responsible for repulsive forces between MTs [340]. The hydrophobic part is very flexible and can be described in terms of a polymer brush model [264]. The interdistance between MTs in the axon is directly related to the effective length of tau proteins [72, 129].

Phosphorylation plays a key role to regulate the various functions of tau or MAP2 proteins. For example, highly phosphorylated tau or MAP2 proteins are more rigid. Then, as a consequence of higher repulsive forces, the interdistance between MTs is increased both in axons and dendrites [264], a feature which can even result in dendritic branching [130]. It is also known that phosphorylation of tau may reduce its affinity to MTs [101, 102].

Since kinesins and tau proteins both bind on the outside of the MT cylinder, it is not unlikely that both proteins interfere with each other. In fact, it has been shown in vitro that kinesin's attachment to the MT is hindered by tau, whereas kinesin is not affected in its motion along the filament once it is bound, i.e., processivity and walking speeds are unaffected [336, 365, 396]. On the other hand, another study found that kinesin had a higher probability of detachment when encountering tau patches on the MT [97]. 
As a consequence of the fact that tau proteins may prevent locally kinesins to bind, tau proteins have been also shown to favor switches of cargos from one microtubule to another one [310], a feature which may be helpful in crowded environments.

The interaction of dyneins with tau proteins are similar to those reported for kinesin: Run lengths are decreased at high concentrations of tau on the MT [336]. In contrast to kinesin, dynein does not detach at encounters of tau but tends to reverse direction, maybe by switching to the diffusive state of motion described above [97]. Altogether, dynein is much less affected by the presence of tau than kinesin and is only seriously impeded in its motion at overexpression of tau [97, 372].

Tau furthermore has a stabilizing effect on MTs by modifying parameters of the dynamic instability of MTs [101]. In the presence of tau, the speed of polymerization is increased while that of depolymerization is decreased. Also, the frequency of catastrophes is lowered and the frequency of rescues is increased. In vitro grown MTs thus reach much higher maximum lengths as a consequence of the action of tau. Still, in normal axons, the MT network is highly dynamic and it is only in some pathologies that the tau concentration becomes high enough to prevent network dynamics.

MTs in axons have been found to be particularly densely decorated by tau proteins at regions of high curvature, suggesting an influence of the mechanical properties [319]. It was also hypothesized that tau has a protective function for MTs against the action of the MT-severing protein katanin [298].

\subsection{Structural defects of the MT network}

Until now, we have not considered that, actually, structural defects of the MT network appear spontaneously in normal axons. Though a large majority of MTs are oriented with their plus-end pointing anterogradely, it seems that 15 to $20 \%$ of the MTs have their plus-end pointing retrogradely [343]. Besides, the whole structure inside the axon includes not only MTs but also neurofilaments and actin filaments. It is not clear whether a minority of filaments oriented differently could not have a strong effect on the overall transport properties, for example by allowing blocked vesicles to go backwards to try another path forward.

Structural defects and axonal transport failure also appear in many neurodegenerative diseases (see [316] for a review). The most prominent example in this respect is Alzheimer's Disease [67, 140, 161, 338]. Axonal swellings caused by accumulations of MAPs, molecular motors, organelles and vesicles have been found in early stages of Alzheimer's Disease, suggesting failure of axonal transport to be linked to the progress of the disease [355]. In particular, tau accumulations in neurofibrillary tangles is a hallmark pathology of Alzheimer's Disease so that overexpression of tau is hypothesized to be implicated in the failure of axonal transport.

As mentioned in the previous section, one of the effects of tau proteins is to lower the attachment rate of kinesins. We have discussed in section 5.3 that binding defects can affect collective motion of particles only if present at almost all sites. This remark may brings a first clue to explain why moderate tau concentrations are not deleterious while tau overexpression is. Indeed, since tau has important stabilizing functions for MTs, it has to be present in normal axons and thus it is desirable that its action as binding defect for kinesins does not affect the overall transport properties if tau is not expressed at extreme concentrations.

A model of the cascade leading from tau overexpression to neuronal degeneration has been proposed by Baas and Qiang [32], based on some experimental results by Mandelkow et al. [246]. As already mentioned, MT-bound tau interferes with the attachment of both motor families, kinesin and dynein, but the effect is more pronounced for kinesin [365, 336]. At normal tau concentrations, this may allow tau to regulate the directionality of axonal transport [97]. In the case of abnormal tau overexpression, this proposed regulation fails and anterograde transport is almost completely suppressed. Baas and Qiang [32], following Mandelkow et al. [246], propose that the neuron reacts to this blocking of the anterograde transport by hyperphosphorylation of tau which causes it to dissociate from the MT filament and thus clears a path for kinesin-driven transport. The hyperphosphorylated tau eventually forms the abnormal filaments mentioned above as a hallmark of Alzheimer's Disease. Additionally to restoring the axonal transport, the loss of tau on the MT makes it more susceptible to severing by katanin causing a disruption of the MT network and neuronal degeneration.

In line with this model, a massive reorientation of the MT network was identified under overexpression of tau in Aplysia neurons [342]. The reorientation might be caused by the elongation of shorter MTs which have been cut by katanin and are brought out of the parallel orientation by steric interaction or molecular motors which pull on the MT fragments. As a consequence of the MT network reorientation, axonal transport is impaired in both directions as swirls in the MT network act as attractive regions for molecular motors so that accumulations of axonal cargos occur as is observed in Alzheimer's Disease. 
The fact that it is possible to reproduce in experiments some swellings similar to those of taupathies, either by using drugs [343] or mutations [344] that impair MT dynamics and vesicular transport, should allow learn more not only for the understanding of neuronal diseases, but more generally of the regulation of intracellular transport.

The knowledge of how an axon regenerates itself when transected brings also some information about how the MT network organizes itself. In [113], an experiment shows the transformation of a transected axonal tip. The experiment allows to visualize the supply in vesicles - a crucial ingredient for the regeneration of the neuron - and the orientation of newly growing MTs. The self-organization of a vesicle trap has been modeled in [147], where the active transport of particles on a filament networks under confinement has been considered. It has been shown that, by applying confining boundary conditions, a self-organization of the network towards a polarized structure is induced, similar to the experimental one, even without explicit regulation and interactions.

\subsection{Global view of model/experiments interplay}

To conclude this review, we would like to give a picture of the current interplay between modeling approaches 17 and experiments. The aim is to understand transport in real cells, but intermediate steps are needed for that. Fig. 37 summarizes various types of approaches to tackle this problem.

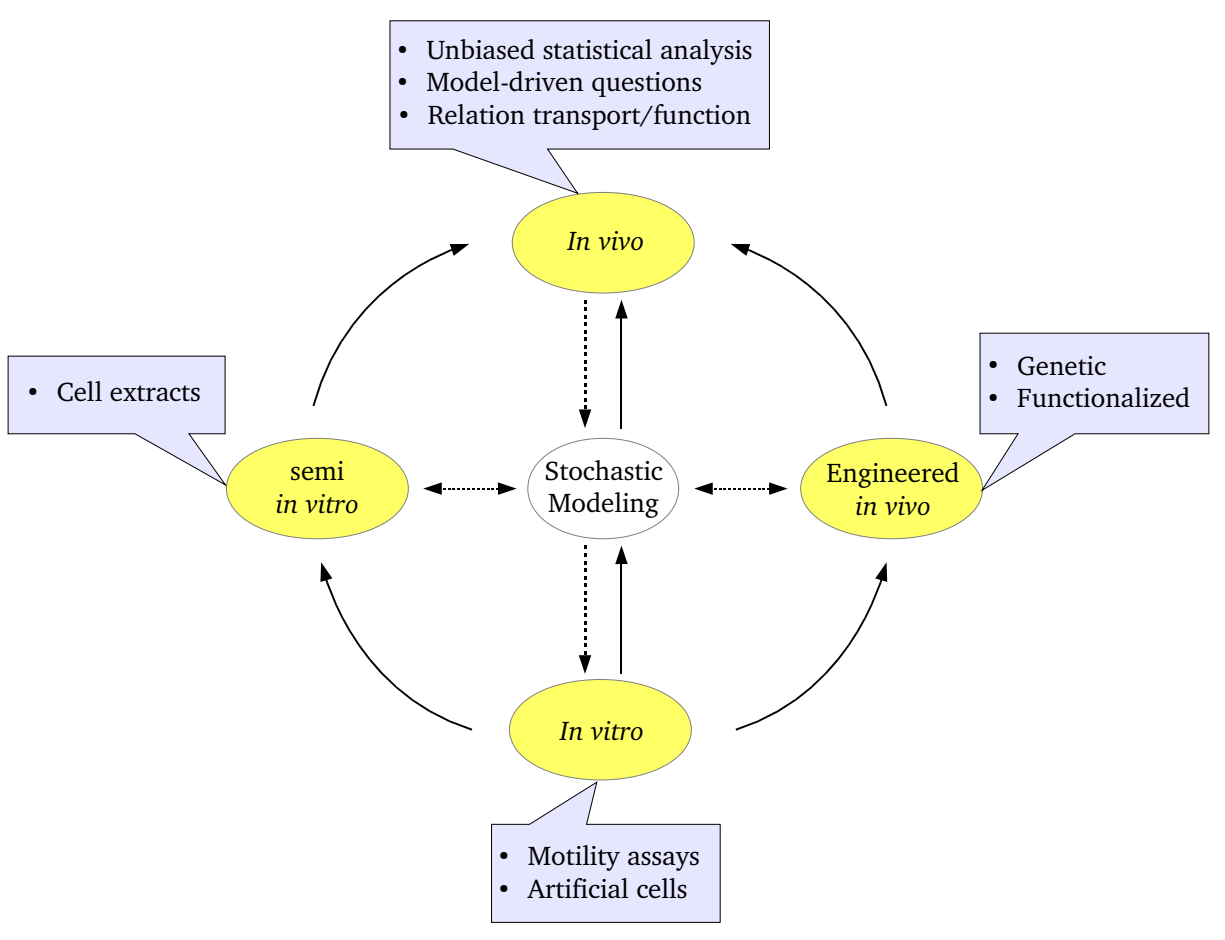

Figure 37: Schematic representation of the different types of experiments, with their interplay with theoretical modeling.

In vitro assays are useful to fully characterize the elementary units of transport, for example motors and MTs. Compared to in vivo experiments, they allow for a better control. One may argue that in vivo, systems are much more complex and cannot be reduced to a sum of elementary units. Still, in vitro experiments are ideal to test the predictive power

\footnotetext{
${ }^{17}$ Note that throughout this review, model or modeling refers to theoretical modeling and simulation of models, in the sense of physicists. In biology, a model can also refer to animal models (for example the mouse is widely considered as a good model to study human diseases), and more generally to all kind of model systems. An example in the frame of the current review is melanophores, which turn out to be a good model system to study organelle transport [23].
} 
of models: building blocks are controlled, and the resulting behavior can be compared between experiments and model simulations.

While motility assays have allowed to fully characterize purified kinesin since a long time, similar results should soon be available for dynein, as it has been recently discovered how to activate in vitro purified mammalian dynein [250]. This also opens the way for building simple cargo-motor complexes [88, 131, 20] on which tug-of-war models' predictions could be tested.

One may argue that in vivo, motors are not isolated but supplemented by many factors which may change their behavior. In order to include these factors, one may not start from purified motors, but directly work with the whole complexes obtained from cell extracts, as done for example in [33, 245] or [156] in semi in vitro experiments.

Of course the final aim is to understand in vivo transport inside cells. Though some measurements can be performed directly in the cell, the interpretation of such measurements is in general difficult as one cannot fully control the setting.

Here we would like to suggest two directions where progress could be made. The first one concerns measurements of cargo trajectories. These trajectories are often cut into runs and pauses. A lot of information is then lost which is of particular relevance for the competition of motors in case of bidirectionally transported cargo. Besides, the definition of runs and pauses requires some threshold-based sorting that may introduce some sensitivity of the results to unwanted features. Another frequent bias of data analysis is to extract extreme events which in fact are rare and not representative. Systematic unbiased statistical study as the one performed for melanosome velocity in [350] would be highly valuable.

Another promising direction of research is to link transport and functions in a more thorough way. Logistics impose some constraints on transport and signaling. Some cell regions may be specialized, e.g., through specific decoration of MTs having an impact on transport properties. More generally, even if models lack firm ground in vivo, they may still help to orient experimental measurements by providing relevant questions.

Another interesting perspective for the understanding of in vivo systems is to modify them. A classical way to do it is via genetic manipulation, that allows either to test a mechanism by modifying one of the actors involved, or to produce cell lines mimicking for example the phenomenology of a given disease.

Genetics can also provide living systems with simpler characteristics, as for example the mutant filamentous fungus Ustilago Maydis studied by G. Steinberg's group [332, 331], which has a much simpler and sparse cytoskeleton than most cells, and besides is quasi one-dimensional (a feature helpful for the modeling task).

New types of engineered cells can now be obtained as well. L. Kapitein et al [182, 184] managed to control the attachment of motors to some cargo (peroxisome), by inserting some molecular switch between the motor and the cargo. Then a simple light exposure can induce attachment of given motors to the cargo. Focusing light on a precise region allows to perform this attachment only locally. Cargos can thus be displaced (using kinesin or dynein) or anchored (using myosin V) at will in specific regions. This opens the possibility to test in vivo various hypothesis, concerning transport itself or its relation with various functions.

Coming back to in vitro systems, we finish our "tour" of the sketch of Fig 37 by noting that, aside the classical motility assays, it is now possible to build artificial cells, i.e. systems obtained from purified units that are combined to build a specific machinery. In M. Dogterom's group for example, some in vitro experiments have been realized, to study how dyneins attached to an artificial cortex can capture MT plus-ends, stabilize them, and mediate forces [218, 217].

Numerous experimental results of all the types described above are expected to come out in the coming years, generating a need for further modeling, and we hope this review will trigger more interest for these systems.

\section{Conclusion}

The application of stochastic transport models to intracellular transport driven by molecular motors stimulated a new and very active branch of research. Compared to typical applications of stochastic transport models, as for example vehicular traffic, several fundamental differences exist: The motion of motor proteins is stepwise such that lattice models are not an approximation but a natural description of the system. Moreover, the number of particles is not conserved - a particle property which is influencing the macroscopic behavior of the system. While stochastic transport models are often defined on a one-dimensional lattice, intracellular transport requires a better understanding of transport on several parallel lanes and on a whole variety of networks. Finally, the networks which carry the active particles themselves are dynamic, a feature that modifies the transport properties. Conversely, the network dynamics and structure can be influenced by the activity of molecular motors. There is growing evidence that the interplay between the cytoskeletal structure and transport is crucial. Implementing into theoretical models the aforementioned characteristics of intracellular transport systems has 
shown their large impact on the transport capacity and particle distribution. However, there is still a need to understand the mechanisms by which this occurs.

Indeed, despite the progress already made, the theoretical understanding of intracellular transport is only in its infancy and still several open problems of fundamental interest exist. Gaining insight in those would help to address questions of strong social impact. The most prominent one is perhaps the relation between perturbation of axonal transport and many neurodegenerative diseases (see [316] for a review). Perturbation of axonal transport may be induced by altering the structure of the MT network or the cargo dynamics. For example, mutations of the protein huntingtin, which influences the directionality of vesicles transport in neurons [81], may cause Huntington's disease [46]. Similarly, transport defects have also been related to Alzheimer's Disease [67, 140, 161, 338].

In order to fully establish the mechanisms that are responsible for neuropathology observations it is necessary to gain a better understanding of unperturbed axonal transport or, to phrase it in a more focused way, of bidirectional motor-driven transport under strong confinement.

We expect that the understanding of intracellular transport processes will dramatically increase by recent progress on the experimental characterization of single motor properties. It is now possible to design rather complex transport systems in vitro in a very controlled way. The results of these experiment can be used to parametrize and validate theoretical models of intracellular transport.

From our point of view the interplay between lattice dynamics and transport is a key issue for biological applications of stochastic transport. Future contributions to this subject may have also strong impact beyond biological applications and involve systems as different as internet or electric networks [163].

\section{Acknowledgments}

We would like to thank our collegues that have contributed to our better knowledge of the field - we thank in particular Philip Greulich, Sarah Klein - and those who have accepted to spend their time to read the manuscript and help us with their valuable advises - in particular Ksenia Astanina, Rosemary Harris and Gunther Schütz. We also thank Daniel Bahr for his help, in particular for some of the figures.

This work was supported by the Deutsche Forschungsgemeinschaft (DFG) within the collaborative research center SFB 1027 and the research training group GRK 1276.

\section{References}

\section{References}

[1] Aamodt, E., Culotti, J., 1986. Microtubules and microtubule-associated proteins from the nematode caenorhabditis elegans: periodic cross-links connect microtubules in vitro. J. Cell. Biol. 103, 23-31.

[2] Adams, D., Schmittmann, B., Zia, R., 2008. Far-from-equilibrium transport with constrained resources. J. Stat. Mech., P06009.

[3] Ahmad, F. J., Baas, P. W., 1995. Microtubules released from the neuronal centrosome are transported into the axon. J. Cell Sci. 108, 2761.

[4] Ahmad, F. J., He, Y., Myers, K. A., Hasaka, T. P., Francis, F., Black, M. M., Baas, P. W., 2006. Effects of dynactin disruption and dynein depletion on axonal microtubules. Traffic 7, 524.

[5] Ahmad, F. J., Yu, W., McNally, F. J., Baas, P. W., 1999. An essential role for katanin in severing microtubules in the neuron. J. Cell Biol. 145, 305.

[6] Akhmanova, A., Dogterom, M., 2011. Kinesins lead aging microtubules to catastrophe. Cell 147, 966-968.

[7] Akhmanova, A., Hoogenraad, C. C., 2005. Microtubule plus-end-tracking proteins: mechanisms and functions. Curr. Opin. Cell Biol. 17, 47-54.

[8] Akhmanova, A., Steinmetz, M. O., 2008. Tracking the ends: a dynamic protein network controls the fate of microtubule tips. Nature Reviews Molecular Cell Biology 9, 309-322.

[9] Al-Bassam, J., Ozer, R. S., Safer, D., Halpain, S., Milligan, R. A., 2002. MAP2 and tau bind longitudinally along the outer ridges of microtubule protofilaments. J. Cell Biol. 157, 1187-1196.

[10] Alberts, B., Johnson, A., Lewis, J., Raff, M., Roberts, K., Walter, P., 2008. Molecular biology of the cell, 5th ed. Garland Science (Taylor\&Francis Group).

[11] Alvarado, J., Sheinman, M., Sharma, A., MacKintosh, F., Koenderink, G., 2013. Molecular motors robustly drive active gels to a critically connected state. Nature Physics 9, 591-597.

[12] Antal, T., Krapivsky, P., Redner, S., 2007. Dynamics of microtubule instabilities. J. of Statistical Mechanics-Theory and Experiment, L05004.

[13] Appert, C., Santen, L., 2001. Boundary induced phase transitions in driven lattice gases with meta-stable states. Phys. Rev. Lett. 86, $2498-2501$.

[14] Appert-Rolland, C., Cividini, J., Hilhorst, H., 2011. Frozen shuffle update for a deterministic totally asymmetric simple exclusion process with open boundaries. J. Stat. Mech., P10013.

[15] Appert-Rolland, C., Cividini, J., Hilhorst, H., 2011. Frozen shuffle update for an asymmetric exclusion process on a ring. J. Stat. Mech., P07009.

[16] Appert-Rolland, C., Cividini, J., Hilhorst, H., 2011. Intersection of two tasep traffic lanes with frozen shuffle update. J. Stat. Mech., P10014. 
[17] Appert-Rolland, C., Hilhorst, H., Schehr, G., 2010. Spontaneous symmetry breaking in a two-lane model for bidirectional overtaking traffic. J. Stat. Mech., P08024.

[18] Arita, C., Ayyer, A., Mallick, K., Prolhac, S., 2011. Recursive structures in the multispecies TASEP. J. of Physics A-Math. and Theo. $44,335004$.

[19] Arita, C., Ayyer, A., Mallick, K., Prolhac, S., 2012. Generalized matrix ansatz in the multispecies exclusion process - the partially asymmetric case. J. Phys. A: Math. Theor. 45, 195001.

[20] Arpag, G., Shastry, S., Hancock, W., Tüzel, E., 2014. Transport by populations of fast and slow kinesins uncovers novel family-dependent motor characteristics important for in vivo function. Biophysical J. 107, 1896-1904.

[21] Ashkin, A., Schütze, K., Dziedzic, J. M., Euteneuer, U., Schliwa, M., 1990. Force generation of organelle transport measured in vivo by an infrared laser trap. Nature 348,346

[22] Ashwin, P., Lin, C., Steinberg, G., 2010. Queueing induced by bidirectional motor motion near the end of a microtubule. Phys. Rev. E $82,051907$.

[23] Aspengren, S., Hedberg, D., Wallin, M., 2007. Melanophores: A model system for neuronal transport and exocytosis? Journal of Neuroscience Research 85, 2591-2600.

[24] Astumian, R., Bier, M., 1994. Fluctuation driven ratchets: Molecular motors. Phys. Rev. Lett. 72, 1766-1769.

[25] Baas, P. W., 1997. Microtubules and axonal growth. Curr. Opin. Cell Biol. 9, 29.

[26] Baas, P. W., 2002. Microtubule transport in the axon. Int. Rev. Cytol. 212, 41-62.

[27] Baas, P. W., Ahmad, F. J., 1992. The plus ends of stable microtubules are the exclusive nucleating structures for microtubules in the axon. J. Cell Biol. 116, 1231.

[28] Baas, P. W., Black, M. M., 1990. Individual microtubules in the axon consist of domains that differ in both composition and stability. J. Cell Biol. $111,495$.

[29] Baas, P. W., Deitch, J. S., Black, M. M., Banker, G. A., 1988. Polarity orientation of microtubules in hippocampal neurons: Uniformity in the axon and nununiformity in the dendrite. Proc. Natl. Acad. Sci. 85, 8335

[30] Baas, P. W., Karabay, A., Qiang, L., 2005. Microtubules cut and run. TRENDS in Cell Biol. 15, 518.

[31] Baas, P. W., Nadar, C. V., Myers, K. A., 2006. Axonal transport of microtubules: the long and short of it. Traffic 7, 490498.

[32] Baas, P. W., Qiang, L., 2005. Neuronal microtubules: when the map is the roadblock. TRENDS in Cell Biol. 15, 183-187.

[33] Barak, P., Rai, A., Dubey, A. K., Rai, P., Mallik, R., 2014. Reconstitution of microtubule-dependent organelle transport. Methods in Enzymology $540,231-248$

[34] Beeg, J., Klumpp, S., Dimova, R., Gracià, R. S., Unger, E., Lipowsky, R., 2008. Transport of beads by several kinesin motors. Biophys. J. 94, 532.

[35] Ben-Naim, E., Krapivsky, P. L., 1999. Maxwell model of traffic flows. Phys. Rev. E 59, 88-97.

[36] Bernhardt, R., Matus, A., 1984. Light and electron microscopic studies of the distribution of microtubule-associated protein 2 in rat brain: A difference between dendritic and axonal cytoskeletons. J. Comp. Neurol. 226, 203.

[37] Bieling, P., Kandels-Lewis, S., Telley, I. A., van Dijk, J., Janke, C., Surrey, T., 2008. CLIP-170 tracks growing microtubule ends by dynamically recognizing composite EB1/tubulin-binding sites. J. Cell Biol. 183, 1223.

[38] Bieling, P., Laan, L., Schek, H., Munteanu, L. E., Sandblad, L., Dogterom, M., Brunner, D., Surrey, T., 2007. Reconstitution of a microtubule plus-end tracking system in vitro. Nature 450 (7172), 1100.

[39] Binder, L. I., Frankfurter, A., Rebhun, L. I., 1985. The distribution of tau in the mammalian central nervous systems. J. Cell Biol. $101,1371$.

[40] Blehm, B. H., Selvin, P. R., 2014. Single-molecule fluorescence and in vivo optical traps: How multiple dyneins and kinesins interact. Chem. Rev. 114, 3335-3352.

[41] Block, S. M., 2007. Kinesin motor mechanics: Binding, stepping, tracking, gating, and limping. Biophys. J. 92, 2986-2995.

[42] Block, S. M., Asbury, C. L., Shaevitz, J. W., Lang, M. J., 2003. Probing the kinesin reaction cycle with a 2D optical force clamp. Proc. Natl. Acad. Sci. 100, 2351.

[43] Blythe, R. A., Evans, M. R., 2007. Nonequilibrium steady states of matrix product form: A solver's guide. J. Phys. A: Math. Theor. 40, R333R441.

[44] Blythe, R. A., Evans, M. R., Colaiori, F., Essler, F. H. L., 2000. Exact solution of a partially asymmetric exclusion model using a deformed oscillator algebra. J. Phys. A: Math. Gen. 33, 2313-2332.

[45] Borodin, A., Ferrari, P., Prahofer, M., 2007. Fluctuations in the discrete TASEP with periodic initial configurations and the Airy process. International Mathematics Research Papers, rpm002.

[46] Borrell-Pages, M., Zala, D., Humbert, S., Saudou, F., 2006. Huntingtons disease: from huntingtin function and dysfunction to therapeutic strategies. Cell Mol. Life Sci. 63, 2642-2660.

[47] Bouzat, S., Falo, F., 2010. The influence of direct motormotor interaction in models for cargo transport by a single team of motors. Phys. Biol. 7 , 046009 .

[48] Bouzat, S., Falo, F., 2011. Tug of war of molecular motors: the effects of uneven load sharing. Phys. Biol. 8, 066010.

[49] Bouzat, S., Levi, V., Bruno, L., 2012. Transport properties of melanosomes along microtubules interpreted by a tug-of-war model with loose mechanical coupling. PLOS One 7, e43599.

[50] Brackley, C. A., Ciandrini, L., Romano, M. C., 2012. Multiple phase transitions in a system of exclusion processes with limited reservoirs of particles and fuel carriers. J. Stat. Mech., P03002.

[51] Brandner, D., Withers, G., 2010. Cultured hippocampal neurons after 2 days in vitro. http://www.cellimagelibrary.org/images/8476.

[52] Brangwynne, C. P., Koenderink, G. H., MacKintosh, F. C., Weitz, D. A., 2008. Nonequilibrium microtubule fluctuations in a model cytoskeleton. Phys. Rev. Lett. 100, 118104.

[53] Brangwynne, C. P., MacKintosh, F. C., Kumar, S., Geisse, N. A., Talbot, J., Mahadevan, L., Parker, K. K., Ingber, D. E., Weitz, D. A., 2006. Microtubules can bear enhanced compressive loads in living cells because of lateral reinforcement. Journal of Cell. Biol. 173, 733.

[54] Brankov, J., Papoyan, V., Poghosyan, V., Priezzhev, V., 2006. The totally asymmetric exclusion process on a ring: Exact relaxation dynamics and associated model of clustering transition. Physica A-Statistical Mechanics and its Applications 368, 471-480.

[55] Brankov, J., Priezzhev, V., Shelest, R., 2004. Generalized determinant solution of the discrete-time totally asymmetric exclusion process and zero-range process. Phys. Rev. E 69, 066136. 
[56] Bressloff, P., Newby, J., 2013. Stochastic models of intracellular transport. Rev. Mod. Phys. 85, 135-196.

[57] Brinkley, B. R., 1985. Microtubule organizing centers. Annu. Rev. Cell Biol. 1, 145.

[58] Brown, A., Jung, P., 2012. A critical reevaluation of the stationary axonal cytoskeleton hypothesis. Cytoskeleton.

[59] Brown, A., Li, Y., Slaughter, T., Black, M. M., 1993. Composite microtubules of the axon: quantitative analysis of tyrosinated and acetylated tubulin along individual axonal microtubules. J. Cell Sci. 104, 339.

[60] Brun, L., Rupp, B., Ward, J. J., Nédélec, F., 2009. A theory of microtubule catastrophes and their regulation. PNAS 106, 21173.

[61] Brunnbauer, M., Dombi, R., Ho, T.-H., Schliwa, M., Rief, M., Okten, Z., 2012. Torque generation of kinesin motors is governed by the stability of the neck domain. Molecular Cell 46, 147-158.

[62] Cameron, D., 2012. Molecular motor struts like drunken sailor. http://hms.harvard.edu/news/molecular-motor-struts-drunken-sailor-1-8-12 8th Jan.

[63] Campas, O., Leduc, C., Bassereau, P., Casademunt, J., Joanny, J.-F., Prost, J., 2008. Coordination of kinesin motors pulling on fluid membranes. Biophysical Journal 94, 5009-5017.

[64] Carter, B., Shubeita, G., Gross, S., 2005. Tracking single particles: a user-friendly quantitative evaluation. Phys. Biol. 2 , 60-72.

[65] Carter, N. J., Cross, R. A., 2005. Mechanics of the kinesin step. Nature 435, 308.

[66] Carvalho, P., Tirnauer, J. S., Pellman, D., 2003. Surfing on microtubule ends. Trends in Cell Biol. 13, $229-237$.

[67] Cash, A. D., Aliev, G., Siedlak, S. L., Nunomura, A., Fujioka, H., Zhu, X., Raina, A. K., Vinters, H. V., Tabaton, M., Johnson, A. B., PaulaBarbosa, M., Avíla, J., Jones, P. K., Castellani, R. J., Smith, M. A., Perry, G., 2003. Microtubule reduction in Alzheimer's disease and aging is independent of tau filament formation. Am. J. Path. 162, 1623

[68] Caspi, A., Granek, R., Elbaum, M., 2002. Diffusion and directed motion in cellular transport. Phys. Rev. E $22,011916$.

[69] Caviston, J. P., Holzbaur, E. L., 2006. Microtubule motors at the intersection of trafficking and transport. Trends in Cell Biology $16,530-537$.

[70] Chai, Y., Lipowsky, R., Klumpp, S., 2009. Transport by molecular motors in the presence of static defects. J. Stat. Phys. 135, 241-260.

[71] Chauwin, J.-F., Ajdari, A., Prost, J., 1994. Force-free motion in asymmetric structures: A mechanism without diffusive steps. Europhys. Lett. 27, 421.

[72] Chen, J., Kanai, Y., Cowan, N., Hirokawa, N., 1992. Projection domains of map2 and tau determine spacings between microtubules in dendrites and axons. Nature 360, 674-676.

[73] Chou, T., Zia, K. M. R. K. P., 2011. Non-equilibrium statistical mechanics: from a paradigmatic model to biological transport. Reports on progress in physics 74,116601

[74] Chowdhury, D., 2013. Stochastic mechano-chemical kinetics of molecular motors: A multidisciplinary enterprise from a physicists perspective. Phys. Reports.

[75] Chowdhury, D., Garai, A., Wang, J.-S., 2008. Traffic of single-headed motor proteins kif1a: Effects of lane changing. Phys. Rev. E 77, 050902(R).

[76] Chowdhury, D., Santen, L., Schadschneider, A., 2000. Statistical physics of vehicular traffic and some related systems. Phys. Reports $329,199$.

[77] Chowdhury, D., Schadschneider, A., Nishinari, K., 2005. Physics of transport and traffic phenomena in biology: from molecular motors and cells to organisms. Physics of Life Reviews 2, 318-352.

[78] Ciandrini, L., Neri, I., Walter, J. C., Dauloudet, O., Parmeggiani, A., 2014. Motor protein traffic regulation by supply-demand balance of resources. Phys. Biol. 11, 056006.

[79] Cividini, J., Appert-Rolland, C., Hilhorst, H., 2013. Diagonal patterns and chevron effect in intersecting traffic flows. Europhys. Lett. 102, 20002.

[80] Cividini, J., Hilhorst, H., Appert-Rolland, C., 2014. Exact domain wall theory for deterministic TASEP with parallel update. J. Phys. A: Math. Theor. (Fast Track Comm.) 47, 222001

[81] Colin, E., Zala, D., Liot, G., Rangone, H., Borrell-Pagès, M., Li, X.-J., Saudou, F., Humbert, S., 2008. Huntingtin phosphorylation acts as a molecular switch for anterograde/retrograde transport in neurons. The EMBO Journal 27, 21242134.

[82] Cook, L. J., Zia, R. K. P., 2009. Feedback and fluctuations in a totally asymmetric simple exclusion process with finite resources. J. Stat. Mech., P02012.

[83] Coy, D. L., Wagenbach, M., Howard, J., 1999. Kinesin takes one 8-nm step for each ATP that it hydrolyzes. The Journal of Biological Chemistry 274, 3667-3671.

[84] David-Pfeuty, T., Erickson, H., Pantaloni, D., 1977. Guanosinetriphosphatase activity of tubulin associated with microtubule assembly. PNAS $74,5372$.

[85] de Gier, J., Nienhuis, B., 1999. Exact stationary state for an asymmetric exclusion process with fully parallel dynamics. Phys. Rev. E 59, 48994911.

[86] Delldot, U., 2008. A stylized depiction of dynein carrying cargo along a microtubule. https://commons.wikimedia.org/wiki/File:Cytoplasmic_dynein.svg.

[87] Dent, E. W., Gertler, F. B., 2003. Cytoskeletal dynamics and transport in growth cone motility and axon guidance. Neuron 40, 209.

[88] Derr, N., Goodman, B., Jungmann, R., Leschziner, A. E., Shih, W. M., Reck-Peterson, S., 2012. Tug-of-war in motor protein ensembles revealed with a programmable DNA origami scaffold. Science 338, 662.

[89] Derrida, B., 1998. An exactly soluble non-equilibrium system: the asymmetric simple exclusion process. Phys. Reports $301,65$.

[90] Derrida, B., Appert, C., 1999. Universal large deviation function of the Kardar-Parisi-Zhang equation in one dimension. J. Stat. Phys. 94, 1-30.

[91] Derrida, B., Domany, E., Mukamel, D., 1992. An exact solution of a one-dimensional asymmetric exclusion model with open boundaries. J. Stat. Phys. 69, 667.

[92] Derrida, B., Evans, M., Hakim, V., Pasquier, V., 1993. Exact solution of a 1d asymmetric exclusion model using a matrix formulation. J. Phys. A 26, 1493.

[93] Desai, A., Mitchison, T. J., 1997. Microtubule polymerization dynamics. Annual Review of Cell and Developmental Biology $13,83-117$.

[94] Desai, A., Verma, S., Mitchison, T., Walczak, C., 1999. Kin I kinesins are microtubule-destabilizing enzymes. Cell 96, 69-78.

[95] Dimitrov, A., Quesnoit, M., Moutel, S., Cantaloube, I., Pous, C., Perez, F., 2008. Detection of GTP-tubulin conformation in vivo reveals a role for GTP remnants in microtubule rescues. Science 322, 1353-1356.

[96] Dixit, R., Barnett, B., Lazarus, J. E., Tokito, M., Goldman, Y. E., Holzbaur, E. L. F., 2009. Microtubule plus-end tracking by CLIP-170 requires EB1. PNAS 106, 492-497.

[97] Dixit, R., Ross, J. L., Goldman, Y. E., Holzbaur, E. L. F., 2008. Differential regulation of dynein and kinesin motor proteins by tau. Science 319 , 
1086-1089.

[98] Dogterom, M., Leibler, S., 1993. Physical aspects of the growth and regulation of microtubule structures. Phys. Rev. Lett. $70,1347$.

[99] Dragestein, K. A., van Cappellen, W. A., van Haren, J., Tsibidis, G. D., Akhmanova, A., Knoch, T. A., Grosveld, F., Galjart, N., 2008. Dynamic behavior of gfp-clip-170 reveals fast protein turnover on microtubule plus ends. J. Cell Biol. 180, 729.

[100] Dreblow, K., Kalchishkova, N., Bohm, K., 2010. Kinesin passing permanent blockages along its protofilament track. Biochemical and Biophysical Research Communications 395, 490-495.

[101] Drechsel, D. N., Hyman, A. A., Cobb, M. H., Kirschner, M. W., 1992. Modulation of the dynamic instability of tubulin assembly by the microtubule-associated protein tau. Mol. Biol. Cell 3, 1141-1154.

[102] Drewes, G., Ebneth, A., Mandelkow, E. M., 1998. MAPs, MARKs and microtubule dynamics. Trends Biochem. Sci. 23, 307-311.

[103] Dudziǹski, M., Schutz, G., 2000. Relaxation spectrum of the asymmetric exclusion process with open boundaries. J. Phys. A: Math. Gen. 33, 8351.

[104] e Silva, M. S., Stuhrmann, B., Betz, T., Koenderink, G., 2014. Time-resolved microrheology of actively remodeling actomyosin networks. New J. Phys. 16, 075010 .

[105] Ebbinghaus, M., 2011. Stochastic modeling of intracellular processes: Bidirectional transport and microtubule dynamics. Ph.D. thesis, Universität des Saarlandes.

[106] Ebbinghaus, M., Appert-Rolland, C., Santen, L., 2010. Bidirectional traffic on microtubules. In: Lecture Notes in Computer Science. Vol. 6350. Springer, pp. 542-551.

[107] Ebbinghaus, M., Appert-Rolland, C., Santen, L., 2010. Bidirectional transport on dynamic networks. Phys. Rev. E 82, 040901(R).

[108] Ebbinghaus, M., Appert-Rolland, C., Santen, L., 2011. Particle interactions and lattice dynamics: Scenarios for efficient bidirectional stochastic transport? J. Stat. Mech., P07004

[109] Ebbinghaus, M., Santen, L., 2009. A model for bidirectional traffic of cytoskeletal motors. J. Stat. Mech., P03030.

[110] Ebbinghaus, M., Santen, L., 2011. Theoretical modeling of aging effects in microtubule dynamics. Biophysical Journal 100, 832-838.

[111] Eisenblätter, B., Santen, L., Schadschneider, A., Schreckenberg, M., 1998. Jamming transition in a cellular automaton model for traffic flow. Phys. Rev. E 57, 1309-1314.

[112] Encalada, S., Szpankowski, L., h. Xia, C., Goldstein, L., 2011. Stable kinesin and dynein assemblies drive the axonal transport of mammalian prion protein vesicles. Cell 144, 551-565.

[113] Erez, H., Malkinson, G., Prager-Khoutorsky, M., Zeeuw, C. I. D., Hoogenraad, C. C., Spira, M. E., 2007. Formation of microtubule-based traps controls the sorting and concentration of vesicles to restricted sites of regenerating neurons after axotomy. J. Cell Biol. 176, $497-507$.

[114] Erlenkämper, C., Kruse, K., 2009. Uncorrelated changes of subunit stability can generate length-dependent disassembly of treadmilling filaments. Phys. Biol. 6, 046016

[115] Evans, M., 1996. Bose-Einstein condensation in disordered exclusion models and relation to traffic flow. Europhysics Letters 36, 13-18.

[116] Evans, M., 1997. Exact steady states of disordered hopping particle models with parallel and ordered sequential dynamics. J. Phys. A: Math Gen. 30, 5669-5685

[117] Evans, M., Ferrari, P., Mallick, K., 2009. Matrix representation of the stationary measure for the multispecies TASEP. J. Stat. Phys. 135, 217-239.

[118] Evans, M., Juhasz, R., Santen, L., 2003. Shock formation in an exclusion process with creation and annihilation. Phys. Rev. E 68, 026117.

[119] Evans, M., Kafri, Y., Sugden, K., Tailleur, J., 2011. Phase diagrams of two-lane driven diffusive systems. J. Stat. Mech., P06009.

[120] Evans, M., Rajewsky, N., Speer, E., 1999. Exact solution of a cellular automaton for traffic. J. Stat. Phys. 95, 45-96.

[121] Fayaz, V., Jafarpour, F. H., Masharian, S. R., Zeraati, S., 2010. One-transit paths and the steady state of a non-equilibrium process in a discretetime update. J. Stat. Mech. P12009.

[122] Fazio, R., Quattrini, A., Bolognesi, A., Bordogna, G., Villa, E., Previtali, S., Canal, N., Nemni, R., 1999. Docetaxel neuropathy: a distal axonopathy. Acta Neuropathol. 98, 651.

[123] Fehr, A. N., Asbury, C. L., Block, S. M., 2008. Kinesin steps do not alternate in size. Biophys. J. 94, L20-L22.

[124] Ferrari, P., Martin, J., 2007. Stationary distributions of multi-type totally asymmetric exclusion processes. Ann. Probab. 35, 807-832.

[125] Fisher, M., Kolomeisky, A., 1999. The force exerted by a molecular motor. PNAS 96, 6597-6602.

[126] Flyvbjerg, H., Holy, T. E., Leibler, S., 1994. Stochastic dynamics of microtubules: A model for caps and catastrophes. Phys. Rev. Lett. 73, 2372.

[127] Forman, D. S., Lynch, K. J., Smith, R. S., 1987. Organelle dynamics in lobster axons: anterograde, retrograde and stationary mitochondria. Brain Res. 412, 96

[128] Fouladvand, M. E., Shaebani, M. R., Sadjadi, Z., 2004. Intelligent controlling simulation of traffic flow in a small city network. Journal of the Physical Society of Japan 73, 3209-3214.

[129] Frappier, T., Georgieff, I., Brown, K., Shelanski, M., 1994. Tau-regulation of microtubule-microtubule spacing and bundling. J. Neurochem. 63, 2288-2294.

[130] Friedrich, P., Aszodi, A., 1991. MAP2 - a sensitive cross-linker and adjustable spacer in dendritic architecture. FEBS Letters 295, 5-9.

[131] Furuta, K., Furuta, A., Toyoshima, Y., Amino, M., Oiwa, K., Kojima, H., 2013. Measuring collective transport by defined numbers of processive and nonprocessive kinesin motors. PNAS 110, 501-506.

[132] Galjart, N., Perez, F., 2003. A plus-end raft to control microtubule dynamics and function. Current Opinion in Cell Biology 15, 48-53.

[133] Gardner, M., Zanic, M., Gell, C., Bormuth, V., Howard, J., 2011. Depolymerizing kinesins kip3 and mcak shape cellular microtubule architecture by differential control of catastrophe. Cell 147, 1092-103.

[134] Gardner, M., Zanic, M., Howard, J., 2013. Microtubule catastrophe and rescue. Curr. Opin. Cell Biol. 25 , 14-22.

[135] Gáspár, I., Szabad, J., 2009. In vivo analysis of mt-based vesicle transport by confocal reflection microscopy. Cell Motility and the Cytoskeleton $66,68-79$.

[136] Gennerich, A., Carter, A. P., Reck-Peterson, S. L., Vale, R., 2007. Force-induced bidirectional stepping of cytoplasmic dynein. Cell 131, 952-965.

[137] Gilbert, S., Webb, M., Brune, M., Johnson, K., 1995. Pathway of processive ATP hydrolysis by kinesin. Nature 373, 671-676.

[138] Gillespie, D. T., 1977. Exact stochastic simulation of coupled chemical reactions. The journal of physical chemistry 81, $2340-2361$.

[139] Gillespie, D. T., 2007. Stochastic simulation of chemical kinetics. Annual Review of Physical Chemistry 58, 35-55.

[140] Goldstein, L., 2001. Kinesin molecular motors: transport pathways, receptors, and human disease. PNAS 98, 6999-7003. 
[141] Gov, N. S., 2009. Physical model for the width distribution of axons. The European Physical Journal E 29, $337-344$.

[142] Govindan, B. S., William B. Spillman, J., 2004. steady states of a microtubule assembly in a confined geometry. Phys. Rev. E 70, 032901.

[143] Greenspan, P., Mayer, E., Fowler, S., 2008. Nile red: a selective fluorescent stain for intracellular lipid droplets. J. Cell Biol. 100, 965-973.

[144] Gregoretti, I., Margolin, G., Alber, M., Goodson, H., 2006. Insights into cytoskeletal behavior from computational modeling of dynamic microtubules in a cell-like environment. J. Cell Sci. 119, 4781-4788.

[145] Greulich, P., 2010. Stochastic modeling of active biological transport in inhomogeneous environments. Ph.D. thesis, Saarland University, Saarbrücken.

[146] Greulich, P., Santen, L., 2010. Active transport and cluster formation on 2D networks. Eur. Phys. J. E 32, 191-208.

[147] Greulich, P., Santen, L., 2011. Boundary-induced orientation of dynamic filament networks and vesicle agglomerations. Phys. Rev. E 84, 060902.

[148] Greulich, P., Schadschneider, A., 2009. Disordered driven lattice gases with boundary reservoirs and Langmuir kinetics. Phys. Rev. E $79,031107$.

[149] Grishchuk, E., 2014. Multiple reversible molecular events at the microtubule tip drive the age-dependent microtubule catastrophes. In: Communication at the EMBO conference "Microtubules: Structure, Regulation and Functions". p. n. 26.

[150] Gross, S., 2003. Dynactin: Coordinating motors with opposite inclinations. Current Biol. 13, R320-R322.

[151] Gross, S. P., 2004. Hither and yon: a review of bi-directional microtubule-based transport. Phys. Biol. 1, R1.

[152] Grzeschik, H., Harris, R. J., Santen, L., 2010. Traffic of cytoskeletal motors with disordered attachment rates. Phys. Rev. E 81, 031929.

[153] Ha, M., den Nijs, M., 2002. Macroscopic car condensation in a parking garage. Phys. Rev. E 66, 036118.

[154] Hancock, W. O., 2014. Bidirectional cargo transport: moving beyond tug of war. Nature Reviews Molecular Cell Biology $15,615$.

[155] Harris, R., Stinchcombe, R., 2005. Ideal and disordered two-lane traffic models. Physica A: Statistical Mechanics and its Applications 354, $582-596$.

[156] Hendricks, A., , Goldman, Y., Holzbaur, E., 2010. Motor coordination via a tug-of-war mechanism drives bidirectional vesicle transport. Current Biology 20, 697-702.

[157] Hendricks, A., Lazarus, J., Perlson, E., Gardner, M., Odde, D., Goldman, Y., Holzbaur, E., 2012. Dynein tethers and stabilizes dynamic microtubule plus ends. Current Biology 22, 632-637.

[158] Hinrichsen, H., 1996. Matrix product ground states for exclusion processes with parallel dynamics. J. Phys. A: Math. Gen. 29 , 3659-3667.

[159] Hirokawa, N., 1982. Cross-linker system between neurofilaments, microtubules and membranous organelles in frog axons revealed by the quickfreeze, deep-etching method. J. Cell. Biol. 94, 129.

[160] Hirokawa, N., 1998. Kinesin and dynein superfamily proteins and the mechanism of organelle transport. Science 279, 519-526.

[161] Hirokawa, N., Takemura, R., 2004. Molecular motors in neuronal development, intracellular transport and diseases. Curr. Opin. Neurobiol. 14, 564-573.

[162] Hollenbeck, P., Saxton, W., 2005. The axonal transport of mitochondria. J. of Cell Science 118, 5411-5419.

[163] Holme, P., Saramäki, J., 2012. Temporal networks. Phys. Reports 519, 97-125.

[164] Hunter, A., Wordeman, L., 2000. How motor proteins influence microtubule polymerization dynamics. J. Cell Sci. 23, 4379-4389.

[165] Iglói, F., Monthus, C., 2005. Strong disorder RG approach of random systems. Phys. Rep. 412, $277-431$.

[166] Jamison, D., Driver, J., Rogers, A., Constantinou, P., Diehl, M., 2010. Two kinesins transport cargo primarily via the action of one motor: Implications for intracellular transport. Biophysical Journal 99, 2967-2977.

[167] Janke, C., Bulinski, J., 2011. Post-translational regulation of the microtubule cytoskeleton: mechanisms and functions. Nature Reviews Molecular Cell Biology 12, 773-786.

[168] Janke, C., Kneussel, M., 2010. Tubulin post-translational modifications: encoding functions on the neuronal microtubule cytoskeleton. Trends in Neurosciences 33, 362-372.

[169] Janowsky, S., Lebowitz, J., 1992. Finite-size effects and shock fluctuations in the asymmetric simple-exclusion process. Phys. Rev. A 45, 618625

[170] Janulevicius, A., van Pelt, J., van Ooyen, A., 2006. Compartment volume influences microtubule dynamic instability: A model study. Biophys. J. 90,788 .

[171] Jelić, A., Appert-Rolland, C., Santen, L., 2012. A bottleneck model for bidirectional transport controlled by fluctuations. Europhys. Lett. 98, 40009.

[172] Jiang, K., Hua, S., Mohan, R., Grigoriev, I., Yau, K., Liu, Q., Katrukha, E., , Altelaar, A., Heck, A., Hoogenraad, C., Akhmanova, A., 2014. Microtubule minus-end stabilization by polymerization-driven CAMSAP deposition. Developmental Cell 28, $295-309$.

[173] Johann, D., Erlenkamper, C., Kruse, K., 2012. Length regulation of active biopolymers by molecular motors. Phys. Rev. Lett. 108, 258103.

[174] Juhasz, R., 2007. Weakly coupled, antiparallel, totally asymmetric simple exclusion processes. Phys. Rev. E 76, 021117.

[175] Juhasz, R., Santen, L., 2004. Dynamics of an exclusion process with creation and annihilation. J. Phys. A: Math. Gen. 37, $3933-3944$.

[176] Jülicher, F., Ajdari, A., Prost, J., 1997. Modeling molecular motors. Rev. Mod. Phys. 69, 1269-1282.

[177] Jülicher, F., Kruse, K., Prost, J., Joanny, J.-F., 2007. Active behavior of the cytoskeleton. Physics Reports 449, 3-28.

[178] Jülicher, F., Prost, J., 1995. Cooperative molecular motors. Phys. Rev. Lett. 75, 2618-2621.

[179] Jülicher, F., Prost, J., 1997. Spontaneous oscillations of collective molecular motors. Phys. Rev. Lett. 78, 45104513.

[180] Jüngst, C., Winterhalder, M. J., Zumbusch, A., 2011. Fast and long term lipid droplet tracking with CARS microscopy. J. Biophotonics not yet assigned, not yet assigned.

[181] Kafri, Y., Lubensky, D., Nelson, D., 2004. Dynamics of molecular motors and polymer translocation with sequence heterogeneity. Biophysical Journal 86, 3373-3391.

[182] Kapitein, L., Schlager, M., van der Zwan, W., Wulf, P., Keijzer, N., Hoogenraad, C., 2010. Probing intracellular motor protein activity using an inducible cargo trafficking assay. Biophys. J. 6, 2143-2152.

[183] Kapitein, L., Hoogenraad, C., 2011. Which way to go? cytoskeletal organization and polarized transport in neurons. Molecular and Cellular Neuroscience 46, 9-20.

[184] Kapitein, L., van Bergeijk, P., Lipka, J., Keijzer, N., Wulf, P., Katrukha, Y., Akhmanova, A., Hoogenraad, C., 2013. Myosin-V opposes microtuble-based cargo transport and drives directional motility on cortical actin. Current Biology 23, 828-834.

[185] Kapon, R., Topchik, A., Mukamel, D., Reich, Z., 2008. A possible mechanism for self-coordination of bidirectional traffic across nuclear pores. 
Physical Biology 5, 036001 .

[186] Karcher, R., Deacon, S., Gelfand, V., 2002. Motor-cargo interactions: the key to transport specificity. Trends Cell Biol. 12, 21-27.

[187] Kardon, J. R., Vale, R. D., 2009. Regulators of the cytoplasmic dynein motor. Nat. Rev. Mol. Cell Biol. 10, 854.

[188] Keime-Guibert, F., Napolitano, M., Delattre, J.-Y., 1998. Neurological complications of radiotherapy and chemotherapy. J. Neurol. $245,695$.

[189] Kirchner, A., Nishinari, K., Schadschneider, A., 2003. Friction effects and clogging in a cellular automaton model for pedestrian dynamics. Phys. Rev. E 67, 056122.

[190] Kirschner, M. W., 1978. Microtubule assembly and nucleation. Int. Rev. Cytol. 54, 1.

[191] Khoromskaia, D., Harris, R., Grosskinsky, S., 2014. Dynamics of non-Markovian exclusion processes. J. Stat. Mech., P12013.

[192] Klein, S., Appert-Rolland, C., Santen, L., 2014. Environmental control of microtubule-based bidirectional cargo-transport. Europhys. Lett. 107, 18004.

[193] Klein, S., Appert-Rolland, C., Santen, L., 2014. Fluctuation effects in bidirectional cargo transport. Eur. Phys. J. Special Topics 223, 3215-3225.

[194] Klein, S., Appert-Rolland, C., Santen, L., 2014. Stochastic modeling of cargo transport by teams of molecular motors. In: Traffic and Granular Flow '13. Springer.

[195] Klein, S., Appert-Rolland, C., Santen, L., 2015. Motility states in bidirectional cargo transport. arXiv:1501.00823.

[196] Klumpp, S., Lipowsky, R., 2003. Traffic of molecular motors through tube-like compartments. J. Stat. Phys. 113, 233-268.

[197] Klumpp, S., Lipowsky, R., 2004. Phase transitions in systems with two species of molecular motors. Europhys. Lett. 66, 90-96.

[198] Klumpp, S., Lipowsky, R., 2005. Active diffusion of motor particles. Phys. Rev. Lett. 95, 268102.

[199] Klumpp, S., Lipowsky, R., 2005. Cooperative cargo transport by several molecular motors. PNAS 102, 17284-17289.

[200] Klumpp, S., Nieuwenhuizen, T., Lipowsky, R., 2005. Self-organized density patterns of molecular motors in arrays of cytoskeletal filaments. Biophysical J. 88, 3118.

[201] Klüpfel, H., 2007. The simulation of crowds at very large events. In: Schadschneider, A., Poschel, T., Kuhne, R., Schreckenberg, M., Wolf, D. (Eds.), Traffic and Granular Flow' 05. pp. 341-346.

[202] Knospe, W., Santen, L., Schadschneider, A., Schreckenberg, M., 1999. Disorder effects in cellular automata for two-lane traffic. Physica A 265 614-633.

[203] Koenderink, G. H., Dogic, Z., Nakamura, F., Bendix, P. M., MacKintosh, F. C., Hartwig, J. H., Stossel, T. P., Weitz, D. A., 2009. An active biopolymer network controlled by molecular motors. PNAS 106, 15192-15197.

[204] Kojima, H., Muto, E., Higuchi, H., Yanagida, T., 1997. Mechanics of single kinesin molecules measured by optical trapping nanometry. Biophys. J. 73, 2012-2022.

[205] Kolomeisky, A., Schütz, G., Kolomeisky, E., Straley, J., 1998. Phase diagram of one-dimensional driven lattice gases with open boundaries. J. Phys. A: Math. Gen. 31, 6911.

[206] Komarova, Y., Akhmanova, A. S., Kojimo, S., Galjart, N., Borisy, G. G., 2002. Cytoplasmic linker proteins promote microtubule rescue in vivo. J. Cell Biol. 159, 589.

[207] Komarova, Y., Lansbergen, G., Galjart, N., Grosveld, F., Borisy, G. G., Akhmanova, A., 2005. EB1 and EB3 control CLIP dissociation from the ends of growing microtubules. Mol. Biol. Cell 16, 5334.

[208] Komarova, Y. A., Vorobjev, I. A., Borisy, G. G., 2002. Life cycle of MTs: persistent growth in the cell interior, asymmetric transition frequencies and effects of the cell boundary. Journal of Cell Science 115, 3527-3539.

[209] Korn, C. B., Klumpp, S., Lipowsky, R., Schwarz, U. S., 2009. Stochastic simulations of cargo transport by processive molecular motors. J. Chem. Phys. 131, 245107.

[210] Krug, J., 1991. Boundary-induced phase transitions in driven diffusive systems. Phys. Rev. Lett. 67, 1882.

[211] Krug, J., Ferrari, P., 1996. Phase transitions in driven diffusive systems with random rates. J. Phys. A: Math. Gen. 29, L465-L471.

[212] Kruse, K., Joanny, J., Jülicher, F., Prost, J., Sekimoto, K., 2005. Generic theory of active polar gels: a paradigm for cytoskeletal dynamics. Eur. Phys. J. E 16, 5-16.

[213] Kulić, I., Brown, A., Kim, H., Kural, C., Blehm, B., Selvin, P., Nelson, P., Gelfand, V., 2008. The role of microtubule movement in bidirectional organelle transport. P.N.A.S. 105, 10011-10016.

[214] Kunwar, A., Mogilner, A., 2010. Robust transport by multiple motors with nonlinear force-velocity relations and stochastic load sharing. Phys. Biol. 7, 016012

[215] Kunwar, A., Tripathy, S. K., Xu, J., Mattson, M. K., Anand, P., Sigua, R., Vershinin, M., McKenney, R. J., Yu, C. C., Mogilner, A., Gross, S. P., 2011. Mechanical stochastic tug-of-war models cannot explain bidirectional lipid-droplet transport. PNAS 108, 18960-18965.

[216] Kural, C., Kim, H., Syed, S., Goshima, G., Gelfand, V. I., Selvin, P. R., 2005. Kinesin and dynein move a peroxisome in vivo: A tug-of-war or coordinated movement? Science 308, 1469.

[217] Laan, L., Pavin, N., Husson, J., Romet-Lemonne, G., van Duin, M., Lopez, M., Vale, R., Julicher, F., Reck-Peterson, S., Dogterom, M., 2012. "cortical" dynein controls microtubule dynamics and length, generating pulling forces that reliably position microtubule asters. Cell 148, 502514.

[218] Laan, L., Roth, S., Dogterom, M., 2012. End-on microtubule-dynein interactions and pulling-based positioning of microtubule organizing centers. Cell Cycle 11, 3750-3757.

[219] Lau, A. W. C., Lacoste, D., Mallick, K., 2007. Nonequilibrium fluctuations and mechanochemical couplings of a molecular motor. Phys. Rev. Lett. 99, 158102

[220] Lee, H.-W., Popkov, V., Kim, D., 1997. Two-way traffic flow: exactly solvable model of traffic jam. J. Phys. A: Math. Gen. $30,8497$.

[221] Leibler, S., Huse, D., 1991. A physical model for motor proteins. C. R. Acad. Sci. Paris III 313, 27-35.

[222] Leibler, S., Huse, D., 1993. Porters versus rowers - a unified stochastic-model of motor proteins. J. Cell. Biology 121, 1357-1368.

[223] Leidel, C., Longoria, R. A., Gutierrez, F. M., Shubeita, G. T., 2012. Measuring molecular motor forces in vivo: Implications for tug-of-war models of bidirectional transport. Biophysical Journal 103, 492-500.

[224] Lenz, M., 2014. Geometrical origins of contractility in disordered actomyosin networks. arXiv:1407.6693 [physics.bio-ph].

[225] Lenz, M., Gardel, M., Dinner, A., 2012. Requirements for contractility in disordered cytoskeletal bundles. New J. Phys. 14, 033037.

[226] Lewis, S. A., Ivanov, I. E., Lee, G.-H., Cowan, N. J., 1989. Organization of microtubules in dendrites and axons is determined by a short 
hydrophobic zipper in microtubule-associated proteins MAP2 and tau. Nature 342, 498-505.

[227] Li, Y., Black, M. M., 1996. Microtubule assembly and turnover in growing axons. J. Neurosci. 16, 531.

[228] Lipowsky, R., Klumpp, S., 2005. "life is motion": multiscale motility of molecular motors. Physica A 352, 53-112.

[229] Lipowsky, R., Klumpp, S., Nieuwenhuizen, T. M., 2001. Random walks of cytoskeletal motors in open and closed compartments. Phys. Rev. Lett. 87, 108101.

[230] Luby-Phelps, K., 1999. Cytoarchitecture and physical properties of cytoplasm: Volume, viscosity. diffusion, intracellular surface area. International Review of Cytology - A Survey of Cell Biology 192, 189-221.

[231] Luby-Phelps, K., Taylor, D. L., Lanni, F., 1986. Probing the structure of cytoplasm. J. Cell Biol. 102, 2015.

[232] Lyman, M., Enquist, L., 2009. Herpesvirus interactions with the host cytoskeleton. J. of Virology 83, $2058-2066$.

[233] Ma, S., Chisholm, R. L., 2002. Cytoplasmic dynein-associated structures move bidirectionally in vivo. J. Cell Sci. 115 , 1453.

[234] Ma, Y., Shakiryanova, D., Vardya, I., Popov, S. V., 2004. Quantitative analysis of microtubule transport in growing nerve processes. Curr. Biol. 14,725 .

[235] MacDonald, C., Gibbs, J., Pipkin, A., 1968. Kinetics of biopolymerization on nucleic acid templates. Biopolymers 6, 1-25.

[236] MacKintosh, F. C., Levine, A. J., 2008. Nonequilibrium mechanics and dynamics of motor-activated gels. Phys. Rev. Lett. $100,018104$.

[237] Maeder, C., Shen, K., Hoogenraad, C., 2014. Axon and dendritic trafficking. Current Opinion in Neurobiology 27, 165-170.

[238] Malgaretti, P., Pagonabarraga, I., Frenkel, D., 2012. Running faster together: Huge speed up of thermal ratchets due to hydrodynamic coupling. Phys. Rev. Lett. 109, 168101

[239] Malgaretti, P., Pagonabarraga, I., Rubi, J. M., 2013. Confined brownian ratchets. J. Chem. Phys. $138,194906$.

[240] Mallick, K., 1996. Shocks in the asymmetry exclusion model with an impurity. J. Phys. A: Math. Gen. 29, 5375-5386.

[241] Mallick, K., 2011. Some exact results for the exclusion process. J. Stat. Mech., P01024.

[242] Mallik, R., Carter, B. C., Lex, S. A., King, S. J., Gross, S. P., 2004. Cytoplasmic dynein functions as a gear in response to load. Nature $427,649$.

[243] Mallik, R., Gross, S. P., 2004. Molecular motors: strategies to get along. Curr Biol. 14, R971-82.

[244] Mallik, R., Petrov, D., Lex, S. A., King, S. J., Gross, S. P., 2005. Building complexity: An in vitro study of cytoplasmic dynein with in vivo implications. Curr. Biol. 15, 2075-2085.

[245] Mallik, R., Rai, A., Barak, P., Rai, A., Kunwar, A., 2013. Teamwork in microtubule motors. Trends in Cell Biology 23, 575-582.

[246] Mandelkow, E.-M., Thies, E., Trinczek, B., Biernat, J., Mandelkow, E., 2004. MARK/PAR1 kinase is a regulator of microtubule-dependent transport in axons. J. Cell Biol. 167, 99.

[247] Margolin, G., Gregoretti, I. V., Goodson, H. V., Alber, M. S., 2006. Analysis of a mesoscopic stochastic model of microtubule dynamic instability. Phys. Rev. E 74, 041920.

[248] Marx, A., Pless, J., Mandelkow, E., Mandelkow, E., 2000. On the rigidity of the cytoskeleton: Are maps crosslinkers or spacers of microtubules? Cell. Mol. Biol. 46, 949-965.

[249] McKay, C. P., 2004. What is life-and how do we search for it in other worlds. PLoS Biol. 2, 1260.

[250] McKenney, R., Huynh, W., Tannenbaum, M., Bhaba, G., Vale, R., 2014. Activation of cytoplasmic dynein motility by dynactin-cargo adapter complexes. Science 345, 337-341.

[251] Meakin, P., Ramanlal, P., Sander, L., Ball, R., 1986. Ballistic deposition on surfaces. Phys. Rev. A 34, 5091

[252] Melbinger, A., Reese, L., Frey, E., 2012. Microtubule length-regulation by molecular motors. Phys. Rev. Lett. 108, 258104.

[253] Miller, K., Heidemann, S., 2008. What is slow axonal transport? Exp Cell Res. 314, 1981-90.

[254] Mitchison, T., Kirschner, M., 1984. Dynamic instability of microtubule growth. Nature 312, $237-242$.

[255] Mitsudo, T., Hayakawa, H., 2005. Synchronization of kinks in the two-lane totally asymmetric simple exclusion process with open boundary conditions. J. Phys. A: Math. Gen 38, 3087-3096.

[256] Mizuno, N., Narita, A., Kon, T., Sutoh, K., Kikkawa, M., 2007. Three-dimensional structure of cytoplasmic dynein bound to microtubules. Proc. Natl. Acad. Sci. 104, 20832.

[257] Mizuno, N., Toba, S., Edamatsu, M., Watai-Nishii, J., Hirokawa, N., Toyoshima, Y., Kikkawa, M., 2004. Dynein and kinesin share an overlapping microtubule-binding site. EMBO Journal 23, 2459-2467.

[258] Moores, C., Yu, M., Guo, J., Beraud, C., Sakowicz, R., Milligan, R., 2002. A mechanism for microtubule depolymerization by KinI kinesins. Molecular Cell 9, 903-909.

[259] Morfini, G., Burns, M., Stenoien, D., Brady, S., 2012. Part i, chapter 8: Axonal transport. In: Brady, S. T., Siegel, G. J., Albers, R. W., Price, D. L. (Eds.), Basic Neurochemistry (Eighth Edition). Elsevier Inc., pp. 146-164.

[260] Moritz, M., Braunfeld, M. B., Sedat, J. W., Alberts, B., Agard, D. A., 1995. Microtubule nucleation by gamma-tubulin-containing rings in the centrosome. Nature 378, 638.

[261] Morrison, E. E., 2007. Action and interactions at microtubule ends. Cell. Mol. Life Sci. 64, 307.

[262] Muhuri, S., Pagonabarraga, I., 2008. Collective vesicle transport on biofilaments carried by competing molecular motors. Europhys. Lett. 84, 58009.

[263] Muhuri, S., Pagonabarraga, I., 2010. Lattice-gas model for active vesicle transport by molecular motors with opposite polarities. Phys. Rev. E 82, 021925.

[264] Mukhopadhyay, R., Kumar, S., Hoh, J., 2004. Molecular mechanisms for organizing the neuronal cytoskeleton. Bioessays 26, $1017-1025$.

[265] Müller, M., Klumpp, S., Lipowsky, R., 2005. Molecular motor traffic in a half-open tube. J. Phys.: Condens. Matter 17, S3839.

[266] Müller, M., Klumpp, S., Lipowsky, R., 2008. Tug-of-war as a cooperative mechanism for bidirectional cargo transport by molecular motors. PNAS 105, 4609-4614.

[267] Müller, M. J. I., Zumbusch, A., 2007. Coherent anti-Stokes Raman scattering microscopy. Chem. Phys. Chem 8, $2157-2170$.

[268] Muto, E., Sakai, H., Kaseda, K., 2005. Long-range cooperative binding of kinesin to a microtubule in the presence of atp. The Journal of Cell Biology 168, 691696.

[269] Myers, K. A., He, Y., Hasaka, T. P., Baas, P. W., 2006. Microtubule transport in the axon: Re-thinking a potential role for the actin cytoskeleton. Neuroscientist 12, 107.

[270] Nagar, A., Majumdar, S. N., Barma, M., 2006. Strong clustering of noninteracting, sliding passive scalars driven by fluctuating surfaces. Phys. 
Rev. E 74, 021124

[271] Nagel, K., Schreckenberg, M., 1992. A cellular automaton model for freeway traffic. J. Phys. I 2, 2221-2229.

[272] Nagy, Z., Appert, C., Santen, L., 2002. Relaxation times in the ASEP model using a DMRG method. J. Stat. Phys. 109, 623-639.

[273] Nieuwenhuizen, T., Klumpp, S., Lipowsky, R., 2002. Walks of molecular motors in two and three dimensions. Europhys. Lett. 58, 468.

[274] Nieuwenhuizen, T., Klumpp, S., Lipowsky, R., 2004. Random walks of molecular motors arising from diffusional encounters with immobilized filaments. Phys. Rev. E 69, 061911.

[275] Nishinari, K., Kanayama, Y., Okada, Y., Greulich, P., Schadschneider, A., Chowdhury, D., 2007. Stochastic modelling and experiments on intracellular transport of single-headed molecular motors. In: Schadschneider, A., Poschel, T., Kuhne, R., Schreckenberg, M., Wolf, D. (Eds.), Traffic and Granular Flow '05. Springer-Verlag Berlin, pp. 263-268.

[276] Nishinari, K., Okada, Y., Schadschneider, A., Chowdhury, D., 2005. Intracellular transport of single-headed molecular motors KIF1A. Phys. Rev. Lett. 95, 118101.

[277] Oakley, B. R., 1994. $\gamma$-tubulin. In: Hyams, J. S., Lloyd, C. W. (Eds.), Microtubules. Wiley-Liss., p. 33.

[278] Oakley, C. E., Oakley, B. R., 1989. Identification of gamma-tubulin, a new member of the tubulin superfamily encoded by mipA gene of $A$. nidulans. Nature 338, 662.

[279] Oberdörster, G., Oberdörster, E., 2005. Nanotoxicology: An emerging discipline evolving from studies of ultrafine particles. Environ Health Perspect. 113, 823-839.

[280] OToole, M., Latham, R., Baqri, R., Miller, K., 2008. Modeling mitochondrial dynamics during in vivo axonal elongation. J. Theor. Biol. 255, $369-377$.

[281] Paggi, P., Lasek, R. J., 1987. Axonal transport of cytoskeletal proteins in oculomotor axons and their residence times in the axon terminals. J. Neurosci. 7, 2397.

[282] Parmeggiani, A., Franosch, T., Frey, E., 2003. Phase coexistence in driven one dimensional transport. Phys. Rev. Lett. 90, 086601.

[283] Parmeggiani, A., Franosch, T., Frey, E., 2004. Totally asymmetric simple exclusion process with Langmuir kinetics. Phys. Rev. E 70, 046101.

[284] Petrov, D., Mallik, R., Shubeita, G., Vershinin, M., Gross, S., Yu, C., 2007. Studying molecular motor-based cargo transport: What is real and what is noise? Biophysical Journal 92, 2953-2963.

[285] Pierobon, P., Frey, E., Franosch, T., 2006. Driven lattice gas of dimers coupled to a bulk reservoir. Phys. Rev. E 74, 031920.

[286] Pierobon, P., Mobilia, M., Kouyos, R., Frey, E., 2006. Bottleneck-induced transitions in a minimal model for intracellular transport. Phys. Rev. E $74,031906$.

[287] Pigorsch, C., Schütz, G., 2000. Shocks in the asymmetric simple exclusion process in a discrete-time update. J. Phys. A: Math. Gen. 33, 79197933.

[288] Pilling, A., Horiuchi, D., Lively, C., Saxton, W., 2006. Kinesin-1 and dynein are the primary motors for fast transport of mitochondria in drosophila motor axons. Molecular Biology of the Cell 17, 2057-2068.

[289] Piper, M., Holt, C., 2004. RNA translation in axons. Annual Review of Cell and Developmental Biology 20, 505-523.

[290] Poghosyan, S., Priezzhev, V., Schutz, G., 2010. Green functions for the TASEP with sublattice parallel update. J. Stat. Mech., P04022.

[291] Poghosyan, V., Priezzhev, V., 2008. The relaxation dynamics of the TASEP with particle-dependent hopping probabilities on a ring. Reports on Mathematical Physics 61, 239-246.

[292] Popkov, V., 2004. Infinite reflections of shock fronts in driven diffusive systems with two species. J. Phys. A: Math. Gen. $37,1545$.

[293] Popkov, V., Peschel, I., 2001. Symmetry breaking and phase coexistence in a driven diffusive two-channel system. Phys. Rev. E $64,026126$.

[294] Popkov, V., Schütz, G., 2003. Shocks and excitation dynamics in a driven diffusive two-channel system. J. Stat. Phys. 112, 523-540.

[295] Popov, S., Poo, M., 1992. Diffusional transport of macromolecules in developing nerve processes. J. Neurosci. 12, 77-85.

[296] Pronina, E., Kolomeisky, A. B., 2004. Two channel totally asymmetric simple exclusion processes. J. Phys. A: Math. Gen. $37,9907$.

[297] Prost, J., Chauwin, J.-F., Peliti, L., Ajdari, A., 1994. Asymmetric pumping of particles. Phys. Rev. Lett. 72, 2652.

[298] Qiang, L., Yu, W., Andreadis, A., Luo, M., Baas, P. W., 2006. Tau protects microtubules in the axon from severing by katanin. J. Neurosci. 26, 3120 .

[299] Qiu, W., Derr, N., Goodman, B., Villa, E., Wu, D., Shih, W., Reck-Peterson, S., 2012. Dynein achieves processive motion using both stochastic and coordinated stepping. Nature Structural \& Molecular Biology 19, 193-200.

[300] Rajewsky, N., Santen, L., Schadschneider, A., Schreckenberg, M., 1998. The asymmetric exclusion process: Comparison of update procedures. J. Stat. Phys. 92, 151-194.

[301] Ray, S., Meyhöfer, E., Milligan, R. A., Howard, J., 1993. Kinesin follows the microtubule's protofilament axis. J. Cell Biol. 121, 1083.

[302] Reck-Peterson, S. L., Yildiz, A., Carter, A. P., Gennerich, A., Zhang, N., Vale, R. D., 2006. Single-molecule analysis of dynein processivity and stepping behavior. Cell 126, 335 .

[303] Reese, L., Melbinger, A., Frey, E., 2011. Crowding of molecular motors determines microtubule depolymerization. Biophysical Journal 101, 2190-2200.

[304] Reichenbach, T., Franosch, T., Frey, E., 2006. Exclusion processes with internal states. Phys. Rev. Lett. 97, 050603.

[305] Reichenbach, T., Franosch, T., Frey, E., 2008. Domain wall delocalization, dynamics and fluctuations in an exclusion process with two internal states. The European Physical Journal E: Soft Matter and Biological Physics 27, 47-56.

[306] Reichenbach, T., Frey, E., Franosch, T., 2007. Traffic jams induced by rare switching events in two-lane transport. New J. Phys. 9, 159.

[307] Reilein, A., Rogers, S., Tuma, M., Gelfand, V., 2001. Regulation of molecular motor proteins. Int. Rev. Cytol. 204, 179-238.

[308] Robert, D., Nguyen, T.-H., Gallet, F., Wilhelm, C., 2010. In vivo determination of fluctuating forces during endosome trafficking using a combination of active and passive microrheology. PloS one 5, e10046.

[309] Rodionov, V. I., Borisy, G. G., 1997. Microtubule treadmilling in vivo. Science 275, 215-218.

[310] Roos, J., Ali, M., Warshaw, D., 2008. Cargo transport: molecular motors navigate a complex cytoskeleton. Curr. Opin. Cell Biol. $20,41-47$.

[311] Roos, W., Campàs, O., Montel, F., Woehlke, G., Spatz, J., Bassereau, P., Cappello, G., 2008. Dynamic kinesin-1 clustering on microtubules due to mutually attractive interactions. Phys. Biol. 5, 046004.

[312] Rosenberg, K. J., Ross, J. L., Feinstein, H. E., Feinstein, S. C., Israelachvili, J., 2008. Complementary dimerization of microtubule-associated tau protein: Implications for microtubule bundling and tau-mediated pathogenesis. Proc. Natl. Acad. Sci. 105, 7445. 
[313] Ross, J. L., Wallace, K., Shuman, H., Goldman, Y. E., Holzbaur, E. L. F., 2006. Processive bidirectional motion of dynein-dynaction complexes in vitro. Nat. Cell Biol. 8, 562

[314] Rousselet, J., Salome, L., Ajdari, A., Prost, J., 1994. Directional motion of brownian particles induced by a periodic asymmetric potential. Nature 370, 446-447.

[315] Roux, A., Cappello, G., Cartaud, J., Prost, J., Goud, B., Bassereau, P., 2002. A minimal system allowing tubulation with molecular motors pulling on giant liposomes. PNAS 99, 5394-5399.

[316] Roy, S., B. Zhang, V.-Y. L., Trojanowski, J., 2005. Axonal transport defects: a common theme in neurodegenerative diseases. Acta Neuropathologica 109, 5-13.

[317] Rzadzinska, A., Schneider, M., Davies, C., Riordan, G., Kachar, B., 2004. An actin molecular treadmill and myosins maintain stereocilia functional architecture and self-renewal. J. Cell Biol. 164, 887-897.

[318] Salman, H., Gil, Y., Granek, R., Elbaum, M., 2002. Microtubules, motor proteins, and anomalous mean squared displacements. Chemical Physics 284, 389-397.

[319] Samsonov, A., Yu, J. Z., Rasenick, M., Popov, S. V., 2004. Tau interaction with microtubules in vivo. J. Cell Sci. 117, 6129.

[320] Santarella, R., Skiniotis, G., Goldie, K., Tittmann, P., Mandelkow, H. G. E., Mandelkow, E., Hoenger, A., 2004. Surface-decoration of microtubules by human tau. J. Mol. Biol. 339, 539-553.

[321] Santen, L., Appert, C., 2002. The asymmetric exclusion process revisited: Fluctuations and dynamics in the domain wall picture. J. Stat. Phys. $106,187-199$.

[322] Saxton, W., Hollenbeck, P., 2012. The axonal transport of mitochondria. J. Cell Sci. 125, 2095-2104

[323] Schadschneider, A., 2002. Traffic flow: a statistical physics point of view. Physica A 313, 153-187.

[324] Schadschneider, A., Schreckenberg, M., 1998. Garden of Eden states in traffic models. J. Phys. A: Math. Gen. 31, L225-L231.

[325] Schadschneider, A., Seyfried, A., 2009. Validation of CA models of pedestrian dynamics with fundamental diagrams. Cybernetics and systems 40, 367-389.

[326] Schiffmann, C., Appert-Rolland, C., Santen, L., 2010. Shock dynamics of two-lane driven lattice gases. J. Stat. Mech., P06002.

[327] Schlager, M., Hoogenraad, C., 2009. Basic mechanisms for recognition and transport of synaptic cargos. Mol Brain 2, 25.

[328] Schliwa, M., 2003. Molecular Motors. Wiley.

[329] Schliwa, M., Woehlke, G., 2003. Molecular motors. Nature 422, 759.

[330] Schrödinger, E., 1992. What is Life? Cambridge University Press.

[331] Schuster, M., Kilaru, S., Fink, G., Collemare, J., Roger, Y., Steinberg, G., 2011. Kinesin-3 and dynein cooperate in long-range retrograde endosome motility along a nonuniform microtubule array. Molecular Biology of the Cell 22, 3645-3657.

[332] Schuster, M., Lipowsky, R., Assmann, M.-A., Lenz, P., Steinberg, G., 2011. Transient binding of dynein controls bidirectional long-range motility of early endosomes. PNAS 108, 3618-3623.

[333] Schütz, G., 1993. Generalized Bethe ansatz solution of a one-dimensional asymmetric exclusion process on a ring with blockage. J. Stat. Phys. $71,471$.

[334] Schütz, G., Domany, E., 1993. Phase transitions in an exactly soluble one-dimensional exclusion process. J. Stat. Phys. 72, $277-296$.

[335] Schuyler, S. C., Pellman, D., 2001. Microtubule "plus-end-tracking proteins": The end is just the beginning. Cell 105, 421-424.

[336] Seitz, A., Kojima, H., Oiwa, K., Mandelkow, E. M., Song, Y. H., Mandelkow, E., 2002. Single-molecule investigation of the intereference between kinesin, tau and MAP2c. EMBO J. 21, 4896.

[337] Seitz, A., Surrey, T., 2006. Processive movement of single kinesins on crowded microtubules visualized using quantum dots. EMBO Journal 25, 267-277.

[338] Seog, D., Lee, D., Lee, S., 2004. Molecular motor proteins of the kinesin superfamily proteins (KIFs): structure, cargo and disease. J. Korean Med. Sci. 19, 1-7.

[339] Shaebani, M., Sadjadi, Z., Rieger, H., Santen, L., 2014. Anomalous diffusion of self-propelled particles in directed random environments. submitted, arXiv:1407.0810

[340] Shahpasand, K., Ahmadian, S., Riazi, G. H., 2008. A possible mechanism for controlling processive transport by microtubule-associated proteins. Neuroscience Research 61, 347350.

[341] Sheetz, M., 1996. Microtubule motor complexes moving membranous organelles. Cell Struct. Funct. 21, 369-373.

[342] Shemesh, O., Erez, H., Ginzburg, I., Spira, M., 2008. Tau-induced traffic jams reflect organelles accumulation at points of microtubule polar mismatching. Traffic 9, 458471.

[343] Shemesh, O., Spira, M., 2010. Paclitaxel induces axonal microtubules polar reconfiguration and impaired organelle transport: implications for the pathogenesis of paclitaxel-induced polyneuropathy. Acta Neuropathologica 119, 235-248.

[344] Shemesh, O. A., Spira, M. E., 2010. Hallmark cellular pathology of alzheimers disease induced by mutant human tau expression in cultured aplysia neurons. Acta Neuropathologica 120, 209-222.

[345] Shubeita, G., Tran, S., Xu, J., Vershinin, M., Cermelli, S., Cotton, S., Welte, M., Gross, S., 2008. Consequences of motor copy number on the intracellular transport of kinesin-1-driven lipid droplets. Cell 135, 1098-1107.

[346] Si, K., Giustetto, M., Etkin, A., Hsu, R., Janisiewicz, A., Miniaci, M. C., Kim, J.-H., Zhu, H., Kandel, E., 2003. A neuronal isoform of CPEB regulates local protein synthesis and stabilizes synapse-specific long-term facilitation in aplysia. Cell 115, 893-904.

[347] Sinnamon, J., Czaplinski, K., 2011. mRNA trafficking and local translation: the Yin and Yang of regulating mRNA localization in neurons. Acta Biochimica et Biophysica Sinica 43, 663-670.

[348] Smith, D., Simmons, R., 2001. Models of motor-assisted transport of intracellular particles. Biophysical Journal 80, $45-68$.

[349] Smith, D. A., Wilson, R. E., 2007. Dynamical pair approximation for cellular automata with shuffle update. J. Phys. A: Math. Theor. 40 (11), 2651-2664.

[350] Snezhko, A., Barlan, K., Aranson, I., Gelfand, V., 2010. Statistics of active transport in Xenopus melanophores cells. Biophys. J. 99, 32163223.

[351] Soppina, V., Rai, A. K., Ramaiya, A. J., Barak, P., Mallik, R., 2009. Tug-of-war between dissimilar teams of microtubule motors regulates transport and fission of endosomes. Proc Natl Acad Sci U S A. 106, 19381-19386.

[352] Standring, S. (Ed.), 2008. Gray’s Anatomy: The Anatomical Basis of Clinical Practice, 40th Edition. Churchill Livingstone. 
[353] Stepanova, T., Slemmer, J., Hoogenraad, C. C., Lansbergen, G., Dortland, B., Zeeuw, C. I. D., van Cappellen, F. G. G., Akhmanova, A., Galjart, N., 2003. Visualization of microtubule growth in cultured neurons via the use of eb3-gfp (end-binding protein 3-green fluorescent protein). The Journal of Neuroscience 23, 2655.

[354] Stiess, M., Maghelli, N., Kapitein, L. C., Gomis-Rüth, S., Wilsch-Bräuninger, M., Hoogenraad, C. C., Tolić-Norrelykke, I. M., Bradke, F., 2010. Axon extension occurs independently of centrosomal microtubule nucleation. Science 327, 704.

[355] Stokin, G. B., Lillo, C., Falzone, T. L., Brusch, R. G., Rockenstein, E., Mount, S. L., Raman, R., Davies, P., Masliah, E., Williams, D. S., Goldstein, L. S. B., 2005. Axonopathy and transport deficits early in the pathogenesis of alzheimer's disease. Science 307,1282

[356] Svoboda, K., Block, S., 1994. Force and velocity measured for single kinesin molecules. Cell 77, $773-784$.

[357] Svoboda, K., Schmidt, C., B, J. S., Block, S., 1993. Direct observation of kinesin stepping by optical trapping interferometry. Nature 365, 721-727.

[358] Tabb, J., Molyneaux, B. J., Cohen, D. L., Kuznetsov, S. A., Langford, G. M., 1998. Transport of ER vesicles on actin filaments in neurons by myosin V. J. Cell Sci. 111, 3221-3234.

[359] Tailleur, J., Evans, M., Kafri, Y., 2009. Nonequilibrium phase transitions in the extraction of membrane tubes by molecular motors. Phys. Rev. Lett. 102, 118109.

[360] Tan, D., Asenjo, A. B., Mennella, V., Sharp, D. J., Sosa, H., 2006. Kinesin-13s form rings around microtubules. Journal of Cell Biology 175, 25-31.

[361] Telley, I., Bieling, P., Surrey, T., 2009. Obstacles on the microtubule reduce the processivity of kinesin-1 in a minimal in vitro system and in cell extract. Biophys. J. 96, 3341-3353.

[362] Tilstra, L., Ernst, M., 1998. Synchronous asymmetric exclusion processes. J. Phys. A: Math. Gen. 31,5033

[363] Tindemans, S. H., Mulder, B. M., 2010. Microtubule length distributions in the presence of protein-induced severing. Phys. Rev. E 81, 031910.

[364] Toba, S., Watanabe, T., Yamaguchi-Okimoto, L., Toyoshima, Y., Higuchi, H., 2006. Overlapping hand-over-hand mechanism of single molecular motility of cytoplasmic dynein. PNAS 103, 5741-5745.

[365] Trinczek, B., Ebneth, A., Mandelkow, E.-M., Mandelkow, E., 1999. Tau regulates the attachment/detachment but not the speed of motors in microtubule-dependent transport of single vesicles and organelles. J. Cell Sci. 112, 2355.

[366] Tripathy, G., Barma, M., 1998. Driven lattice gases with quenched disorder: Exact results and different macroscopic regimes. Phys. Rev. E 58, 1911-1926.

[367] Tsekouras, K., Kolomeisky, A., 2008. Parallel coupling of symmetric and asymmetric exclusion processes. J. of Physics A - Math. and Theo. 41, 465001.

[368] Turci, F., Parmeggiani, A., Pitard, E., Romano, M. C., Ciandrini, L., 2013. Transport on a lattice with dynamical defects. Phys. Rev. E 87, 012705.

[369] Vale, R., 2003. The molecular motor toolbox for intracellular transport. Cell 112, 467-480.

[370] Vale, R., Milligan, R., 2000. The way things move: Looking under the hood of molecular motor proteins. Science 288, 88-95.

[371] Vale, R. D., Fletterick, R. J., 1997. The design plan of kinesin motors. Annu. Rev. Cell Dev. Biol. 13, $745-777$.

[372] Vershinin, M., Xu, J., Razafsky, D. S., King, S. J., Gross, S. P., 2008. Tuning microtubule-based transport through filamentous maps: The problem of dynein. Traffic 9, 882 .

[373] Vicsek, T., Zafeiris, A., 2012. Collective motion. Physics Reports 517 (3-4), 71-140.

[374] Viel, A., Lue, R. A., Liebler, J., Biovisions project - inner life series (see around time 3'20 for microtubule dynamics and kinesin behavior). http://multimedia.mcb.harvard.edu.

[375] Vilfan, A., Frey, E., Schwabl, F., Thormählen, M., Song, Y.-H., Mandelkow, E., 2001. Dynamics and cooperativity of microtubule decoration by the motor protein kinesin. Journal of Molecular Biology 312, 1011-1026.

[376] Visscher, K., Schnitzer, M., Block, S., 1999. Single kinesin molecules studied with a molecular force clamp. Nature 400, 184-189.

[377] Vuppalanchi, D., Willis, D., Twiss, J., 2009. Regulation of mRNA transport and translation in axons. Results Probl. Cell Differ. 48, 193-224.

[378] Wacker, I., Kaether, C., Kromer, A., Migala, A., Almers, W., Gerdes, H., 1997. Microtubule-dependent transport of secretory vesicles visualized in real time with a GFP-tagged secretory protein. J. Cell Sci. 110, 1453-1463.

[379] Walker, R. A., Inoué, S., Salmon, E. D., 1989. Asymmetric behavior of severed microtubule ends after ultraviolet-microbeam irradiation of individual microtubules in vitro. J. Cell Biol. 108, 931-937.

[380] Walker, R. A., O’Brien, E. T., Pryer, N. K., Soboeiro, M. F., Voter, W. A., Erickson, H. P., Salmon, E. D., 1988. Dynamic instability of individual microtubules analyzed by video light microscopy: Rate constants and transition frequencies. J. Cell Biol. $107,1437$.

[381] Wang, G., Sevick, E., Mittag, E., Searles, D., Evans, D., 2002. Experimental demonstration of violations of the second law of thermodynamics for small systems and short time scales. Phys. Rev. Lett. 89, 050601.

[382] Wang, R., Jiang, R., Liu, M., Liu, J., Wu, Q.-S., 2007. Effects of langmuir kinetics of two-lane totally asymmetric exclusion processes in protein traffic. Int. J. Mod. Phys. C 18, 1483-1496.

[383] Wang, Z., Khan, S., Sheetz, M. P., 1995. Single cytoplasmic dynein molecule movements: Characterization and comparison with kinesin. Biophys. J. 69, 2011-2023.

[384] Wang, Z., Sheetz, M. P., 1999. One-dimensional diffusion on microtubules of particles coated with cytoplasmic dynein and immunoglobulins. Cell Struct. Funct. 24, 373.

[385] Watanabe, T., Thayil, A., Jesacher, A., Grieve, K., Debarre, D., Wilson, T., Booth, M., Srinivas, S., 2010. Characterisation of the dynamic behaviour of lipid droplets in the early mouse embryo using adaptive harmonic generation microscopy. BMC Cell Biology 11,38

[386] Waterman-Storer, C., Salmon, E., 1997. Actomyosin-based retrograde flow of microtubules in the lamella of migrating epithelial cells influences microtubule dynamic instability and turnover and is associated with microtubule breakage and treadmilling. J. Cell. Biol. 139, 417-434.

[387] Wells, A., 1999. Myosin VI is an actin-based motor that moves backwards. Nature 401, 505-508.

[388] Welte, M. A., 2004. Bidirectional transport along microtubules. Curr. Biol. 14, R525-R537.

[389] Welte, M. A., 2009. Fat on the move: intracellular motion of lipid droplets. Biochem. Soc. Trans. 37, 991-996.

[390] Wölki, M., Schadschneider, A., Schreckenberg, M., 2006. Asymmetric exclusion processes with shuffled dynamics. J. Phys. A-Math. Gen. 39, $33-44$

[391] Wölki, M., Schadschneider, A., Schreckenberg, M., 2007. Asymmetric exclusion processes with non-factorizing steady states. In: Schadschnei- 
der, A., Poschel, T., Kuhne, R., Schreckenberg, M., Wolf, D. (Eds.), Traffic and Granular Flow’ 05. pp. 473-479.

[392] Wölki, M., Schreckenberg, M., 2009. Exact matrix-product states for parallel dynamics: open boundaries and excess mass on the ring. J. Stat. Mech., P05014.

[393] Yildiz, A., Forkey, J., McKinney, S., Ha, T., Goldman, Y., Selvin, P., 2003. Myosin V walks hand-over-hand: single fluorophore imaging with 1.5-nm localization. Science 300, 2061-2065.

[394] Yildiz, A., Tomishige, M., Vale, R., Selvin, P., 2004. Kinesin walks hand-over-hand. Science 303, 676-678.

[395] Yu, W., Baas, P. W., 1994. Changes in microtubule number and length during axon differentiation. The Journal of Neuroscience 14, $2818-2829$.

[396] Yuan, A., Kumar, A., Peterhoff, C., Duff, K., Nixon, R. A., 2008. Axonal transport rates In Vivo are unaffected by tau deletion or overexpression in mice. J. Neurosci. 28, 1682.

[397] Yule, G., 1924. A mathematical theory of evolution, based on the conclusions of Dr. J. C. Willis. Philos. Trans. Roy. Soc. London Ser. B 213, $21-87$.

[398] Zala, D., Hinckelmann, M.-V., Yu, H., da Cunha, M. M. L., Liot, G., Cordelières, F., Marco, S., Saudou, F., 2013. Vesicular glycolysis provides on-board energy for fast axonal transport. Cell 152, 479-491.

[399] Zhang, Y., Fisher, M. E., 2010. Dynamics of the tug-of-war model for cellular transport. Phys. Rev. E 82, 011923.

[400] Zheng, Y., Wong, M. L., Alberts, B., Mitchison, T., 1995. Nucleation of microtubule assembly by a gamma-tubulin-containing ring complex. Nature 378, 578

[401] Zia, R., Dong, J., Schmittmann, B., 2011. Modeling translation in protein synthesis with TASEP: A tutorial and recent developments. J. Stat. Phys. 144, 405-428. 